$$
\text { PANEL DATA }
$$

Gary Chamberlain

working Paper NO. 913

NATIONAL BUREAU OF ECONOMIC RESEARCH

1050 Masaachusetts Avenue

Cambridge MA 02138

June 1982

The research reported here is part of the NBER's research program in Labor Studies. Any opinions expressed are those of the author and not those of the National Bureau of Economic Research. 


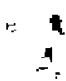

$\therefore$

: 


\section{Panel Data}

\section{ABSTRACT}

We conslder linear predictor definitions of noncausality or strict exogeneity and show that it $\mathbf{1}$ s restrictive to assert that there exists a timeinvariant latent variable $\mathbf{c}$ such that $\mathbf{x}$ is strictly exogenous conditional on $\mathbf{c}$. A restriction of this sort is necessary to justify standard techniques for controlling for unobserved individual effects. There is a parallel analysis for multivariate probit models, but now the distributional assumption for the individual effects is restrictive. This restriction can be avoided by using a conditional likelihood analysis in a logit model. Some of these ideas are illustrated by estimating union wage effects for a sample of young Men in the National Longitudinal Survey. The results indicate that the lags and leads could have been generated just by an unobserved individual effect, which gives some support for analysis of covariance-type estimates. These estimates indicate a substantial omitted variable bias. We also present estimates of a model of female labor force participation, focusing on the relationship between participation and fertility. Unlike the wage example, there is evidence against conditional strict exogenelty; if we ignore this evidence, the probit and logit approaches give conflicting results.

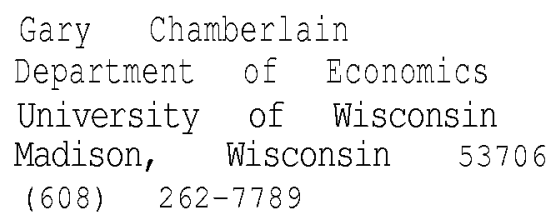




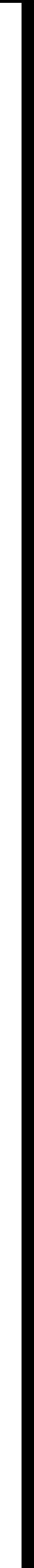


PANEL DATA

TABLE OF CONTENTS

1. INTRODUCTION AND SUMMARY

2. SPECIFICATION AND IDENTIFICATION: LINEAR MODELS 12

2.1 A Production Function Exomple 12

2.2 Fired Effects and Incidental Parameters 14

2.3 Random Effects and Specification Analyais 15

2.4 A Consumer Demand Example 19

2.4.a Certainty

2.4. b Uncertainty

2.4.c Labor Supply

2.5 strict Exogeneity Conditional on a Latent Variable 23

2.6 Lagged De\&dent Variables 26

2.7 Residual Covarionoes: Heteroskedasticity and Serial Correlation

2.7.a Heteroskedasticity

2.7.b Semial Comelation

3. SPECIFICATION AND IDENTIFICATION: NONLINEAR MODELS 32

3.1 A Rondom Effects Frobit Model 32

3.2 A Fixed Effects Logit Model: Conditional Likelihood 37

3. 3 Serial Correlation and Lagged Dependent Variables 43

3.4 Duration Modets 
4. INFERENCE 55

4.1 The Estimation of Linear Predictors 56

4.2 Imposing Restrictions: The Minimum Distance Estimator 58

4.3 Simultaneous Equations: A Generalization of Two-and Three-Stage Least Squares

4.4 Asymptotic Efficiency: A Comparison with the QuasiMaximum Like i ihood Estimator

4.5 Multivariate Probit Models 74

5. EMPIRICAL APPLICATIONS

5.1 Linear Models: Union Wage Effects 78

5.2 Nontinear Models: Labor Force Participation a5

6. CONCLUSION 90

$\begin{array}{lr}\text { APPENDIX } & 102\end{array}$

$\begin{array}{lr}\text { FOOTNOTES } & 104\end{array}$

$\begin{array}{lr}\text { REFERENCES } & 109\end{array}$ 


\section{INTRODUCTION AND SUMMARY}

The paper has four parts: the specification of linear models; the specification of nonlinear models; statistical inference; and empirical applications. The choice of topics is highly selective. We shall focus on a few problems and try to develop solutions in some detail.

The discussion of linear models begins with the following specification:

$$
y_{1 t}=\beta x_{1 t}+c_{i} \cdot u_{1 t} \text {, }
$$

$$
E\left(u_{1 t} \mid x_{1 I}, \ldots . x_{1 T}, c_{1}\right)=0 \quad\left(i=1, \ldots, N_{i} t-1, \ldots, T\right)
$$

For example, in a panel of farms observed over several years, suppose that $y_{i t}$ is a measure of the output of the $i \frac{\text { th }}{\text { farm in the } t \text { th }}$ season, $\mathbf{x}_{\text {it }}$ is a measured input that varies over time, $c_{i}$ is an unmeasured, fixed input reflecting soil quality and other characteristics of the farm's location, and $u_{1 t}$ reflects unmeasured inputs that vary over time such as rainfall. Suppose that data is available on $\left(\mathbf{x}_{\mathbf{i} \mathbf{I}}, \ldots . . \mathbf{x}_{\mathbf{i T}}, \mathbf{y}_{\mathbf{i I}}, \ldots . . \mathbf{y}_{\mathbf{i T}}\right)$ for each of a large number of units, but $c_{1}$ is not observed. A crosssection regression of $\boldsymbol{y}_{\boldsymbol{i l}}$ on $\boldsymbol{x}_{\boldsymbol{i l}}$ will give a biased estimate of $\boldsymbol{B}$ if $\boldsymbol{c}$ is correlated with $x$, as we would expect it to be in the production function example. Furthermore, with a single cross section, there may be no internal evidence of this bias. If $\mathrm{T}>1$, we can solve this problem given the assumption in (1.2). The change in $y$ satisfies

$$
E\left(y_{12}-y_{11} \mid x_{12}-x_{11}\right)=\beta\left(x_{12}-x_{11}\right)
$$

and the least squares regression of $y_{12}-y_{11}$ on $x_{12}-x_{11}$ provides a 
consistent estimator of $B($ as $\rightarrow+\infty)$ if the change in $\mathrm{x}$ has sufficient variation. A generalization of this estimator when $T>2$ can be obtained from a least squares regression with individual specific intercepts.

The restriction in (1.2) is necessary for this result. For example, consider the following autoregressive specification:

$$
\begin{aligned}
& y_{i t}=\beta y_{1, t-1}+s_{i}+u_{1 t}, \\
& E\left(u_{1 t} \mid y_{1, t-1}, c_{1}\right)=0 .
\end{aligned}
$$

It is clear that a regression of $\mathrm{y}_{\mathbf{i t}}-\mathrm{y}_{\mathbf{1} \mathbf{t - 1}}$ on $\mathrm{y}_{\mathbf{i}, \mathbf{t}-\mathbf{1}}-\mathrm{y}_{\mathbf{1}, \mathbf{t}-\mathbf{2}}$ will not provide a consistent estimator of $\beta$, since $\mathbf{u}_{\mathbf{i t}}-\mathbf{u}_{\mathbf{i}, \mathbf{t}-\mathbf{1}}$ is correlated with $y_{i, t-1}=y_{i, t-2}$. Hence it is not sufficient to assume that

$$
E\left(u_{i t} \mid x_{i t}, c_{i}\right)=J
$$

Much of our discussion will be directed at testing the stronger restriction in $(1.2)$.

Consider the (minimm mean-square error) linear predictor of $\boldsymbol{c}_{\mathbf{i}}$ conditional on $\mathbf{x}_{\mathbf{1 1}}, \ldots \cdot \mathbf{x}_{\mathbf{i T}}$ :

$$
E *\left(\varepsilon_{1} \mid x_{11}, \ldots . x_{1 T}\right)=\eta+\lambda_{1} x_{11}+\ldots+\lambda_{T} x_{1 T} .
$$

Given the assumptions that variances are finite and that the distribution of $\left(\mathbf{x}_{\mathbf{i l}}, \ldots, \mathbf{x}_{\mathbf{i} \mathbf{T}}, \mathbf{c}_{\mathbf{i}}\right)$ do as not depend upon $i$, there are no additional restrictions in (1.3); it is simply notation for the linear predictor. Now consider the linear predictor of $\mathbf{y}_{\mathbf{1 t}}$ given $\mathbf{x}_{\mathbf{i l}}, \ldots . . \mathbf{x}_{\mathbf{i T}}$ :

$$
E *\left(y_{1 t} \mid x_{11}, \ldots . . x_{i T}\right)=\zeta_{t}+\pi_{t I} x_{11}+\ldots+\pi_{t T} x_{i T}
$$


Form the $T \times T$ matrix $I$ with $\pi_{t s}$ as the $(t, s)$ element. Then the restriction in (1.2) implies that $\pi$ has a distinctive structure:

$$
\underset{v}{I}=\beta I+\ell \stackrel{\alpha}{\prime}^{\prime},
$$

where $I$ is the $I \times T$ identity matrix, $l$ is a $\mathbb{I} \times 1$ vector of ones, and

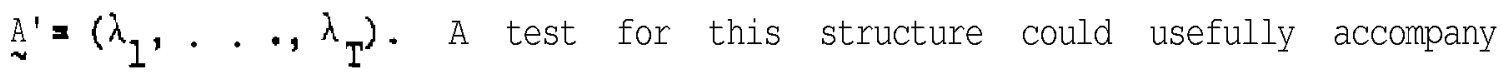
estimators of $\beta$ based on change regressions or on regressions with individual specific intercepts. Moreover, this formulation suggests an alternative estimator for $\beta$, which is developed in the inference section. This test is an exogeneity test and it is useful to relate it to Granger (1969) and Sims (1972) causality. The novel feature is that we are testing for noncausality conditional on a latent variable. Suppose that $\mathrm{t}=1$ is the first period of the individual's (economic) life. Within the linear predictor context, a Granger definition of " $y$ does not cause $x$ conditional on a latent variable $c^{\prime \prime}$ is

$$
\begin{aligned}
& E^{*}\left(x_{i, t+1} \mid x_{i 1}, \ldots, x_{i t}, y_{11}, \ldots, y_{i t}, c_{i}\right) \\
& =E^{*}\left(x_{i, t+1} \mid x_{11}, \ldots, x_{i t}, c_{i}\right) \quad(t=1,2, \ldots) .
\end{aligned}
$$

A Sims definition is

$$
E *\left(y_{i t} \mid x_{i 1}, x_{12}, \ldots, c_{1}\right)=E *\left(y_{1 t} \mid x_{i 1}, \ldots, x_{i t}, c_{1}\right) \quad(t=1,2, \ldots)
$$

In fact, these two definitions imply identical restrictions on the covariance matrix of $\left(x_{i 1}, \ldots, x_{i T}, y_{i l}, \ldots, y_{i T}\right)$. The sims form fits directly into the $\underset{\sim}{\mathbb{\pi}}$ matrix framework and implies the following restrictions:

$$
\underline{I}=\underset{N}{B}+\underline{\lambda}^{\prime}
$$


where $B$ is a lower triangular matrix and $Y$ is a $\mathbb{I} \times 1$ vector.

We show how these nonlinear restrictions can be transformed into linear restrictions on a standard simultaneous equations model. we show also how a $\gamma \lambda^{\prime}$ term can arise in an autoregressive model from the projection of an initial condition onto the $x^{\prime} s$.

In Sectian 3 we use a multivariate probit model to illustrate the new issues that arise in models that are noninnear in the variables. Consider the following specification:

$$
\begin{aligned}
\tilde{y}_{i t} & =\beta x_{i t}+c_{i}+u_{i t}, \\
y_{i t} & : 1 \text { if } \tilde{y}_{i t} \geq 0, \\
& =0 \text { otherwise } \quad(i=1, \ldots . N ; t=1, \ldots . T),
\end{aligned}
$$

where, condi ional on $\mathbf{x}_{i l}, \ldots, \mathbf{x}_{\mathbf{i T}}, \boldsymbol{c}_{\mathbf{1}}$, the distribution of $\left(\mathbf{u}_{\mathbf{i l}}, \ldots . . \mathbf{u}_{\mathrm{T}}\right)$ is multivarlate normal $\left(\mathbb{N}(\underset{\sim}{0}, \Sigma)\right.$ ) with mean $\underset{\sim}{0}$ and covariance matrix $\Sigma=\left(\sigma_{j k}\right)$. We observe $\left(\mathbf{x}_{\mathbf{i l}}, \ldots . \mathbf{x}_{\mathbf{i T}}, \mathbf{y}_{\mathbf{i l}}, \ldots \mathbf{y}_{\mathbf{i T}}\right)$ for a large number of individuals, but we do not observe $\mathbf{c}_{\mathbf{i}}$. For example, in the reduced form of a labor force participation model, $y_{1 t}$ can indicate whether or not the $i$ th individual wnrked during period $t, \mathbf{x}_{\mathbf{i t}}$ can be a measure of the presence of young children, and $\boldsymbol{c}_{\mathbf{i}}$ can capture unmeasured characteristics of the individual that are stable at least over the sample period. In the certainty model of Heckman and MaCurdy (1980), $c_{1}$ is generated by the single life-time budget constraint.

$$
\text { If we treat the } \boldsymbol{c}_{\mathbf{i}} \text { as parameters to be estimated, then there is a }
$$

severe incidental parameter problem. The consistency of the maximum 
likelihood estimator requires that $\mathrm{T} \rightarrow \infty$, but we want to do asymptotic inference with $\boldsymbol{N} \rightarrow \infty$ for fixed $T$, which reflects the sample sizes in the panel data sets we are most interested in. So we consider a random. effects estimator, which is based on the following specification for the distribution of $c$ conditional on $x$ :

$$
c_{i}=\eta+\lambda_{1} x_{i 1}+\ldots+\lambda_{T} x_{i T}+v_{i}
$$

where the distribution of $\mathbf{v}_{\mathbf{1}}$ conditional on $\mathbf{x}_{\mathbf{1 I}}, \ldots . . \mathbf{x}_{\mathbf{i T}}$ is $\mathbf{N}\left(0, \sigma_{\mathrm{v}}^{2}\right)$. This is similar to our specification in (1.3) for the linear model, but there is an important difference; (1.3) was just notation for the linear predictor, whereas (1.4) embodies substantive restrictions. We are assuming that the regression function of $c$ on the $x^{\prime} s$ is linear and that the residual variation is homoskedastic and normal. Given these assumptions, our analysis runs parallel to the linear case. There is a matrix II of multivarlate probit coefficients which has the following structure:

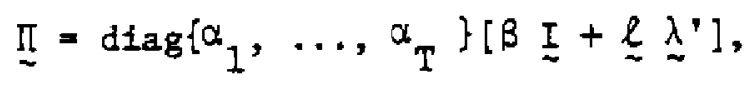

where $\operatorname{diag}\left\{\alpha_{1}, \ldots . \alpha_{\mathrm{T}}\right\}$ is a diagonal matrix of normalization factors with $\alpha_{t}=\left(\sigma_{t t}+\sigma_{v}^{2}\right)^{-\frac{1}{2}}$. We can impose these restrictions to obtain an estimator of $\alpha_{t} \beta$ which is consistent as $N \rightarrow \infty$ for fixed $T$. We can also test whether $\underset{\sim}{\mathbb{I}}$ in fact has this structure.

A quite different treatment of the incidental parameter problem is possible with a logit functional form for $\mathbf{P}\left(\mathbf{y}_{\mathbf{i t}} \mathbf{= 1} \mid \mathbf{x}_{\mathbf{1 t}}, \mathrm{ci}\right)$. The sum $\Sigma_{t=1}^{T} y_{i t}$ provides a sufficient statistic for ci. Hence we can use the distribution of $\mathbf{y}_{\mathbf{i l}}, \ldots . . \mathbf{y}_{\mathbf{i T}}$ conditional on $\mathbf{x}_{\mathbf{1 1}}, \ldots . . \mathbf{x}_{\mathbf{i T}}, \Sigma_{\mathbf{t}} \mathbf{y}_{\mathbf{1 t}}$ 
to obtain a conditional likelihood function that does not depend upon $\boldsymbol{c}_{i}$. Maximizing it with respect to $\beta$ provides an estimator that is consistent as $\mathrm{N} \rightarrow \infty$ for fixed $\mathrm{T}$, and the other standard properties for maximum likelihood hold as well. The power of the procedure is that it places no restrictions on the conditional distribution of $c$ given $x$. It is perhaps the closest analog to the change regression in the linear model. A shortcoming is that the residual covariance matrix is constrained to be equicorrelated. Just as in the probit model, a key assumption is

$$
P\left(y_{i t}=1 \mid x_{i l}, \ldots . . . x_{i T}, c_{1}\right)=P\left(y_{i t}=1 \mid x_{i t}, c_{i}\right),
$$

and we discuss how it can be tested.

It is natural to ask whether (1.5) is testable without imposing the various functional form restrictions that underlie our tests in the probit and logit cases. First, some definitions. Suppose that $t=1$ is the initial period of the individual's (economic) life; an extension of Sims' condition for $\mathbf{x}$ to be strictly exogenous is that $\mathbf{y}_{\mathbf{t}}$ is independent of $\mathbf{x}_{\mathbf{t}+\mathbf{1}}, \mathbf{x}_{\mathbf{t + 2}}, \cdots$ conditional on $\mathbf{x}_{\mathbf{1}}, \ldots, \mathbf{x}_{t}$. An extension of Granger's condition for "y does not cause $\mathbf{x}^{\prime \prime}$ is that $\mathbf{x}_{\mathbf{t}+\mathbf{1}}$ is independent of $\mathrm{yl}_{\mathbf{l}} . . . \mathbf{y}_{\mathbf{t}}$ conditional on $\mathbf{x}_{1}, \ldots . \mathbf{x}_{t^{\prime}}$ Unlike the linear predictor case, now strict exogenefty is weaker than noncausality. Noncausality requires that $y_{t}$ be independent of $x_{t+1}, x_{t+2}, \ldots$ conditional on $x_{1}, \ldots, x_{t}$ and on $y_{1} \ldots . . y_{t-1}$. If $x$ is strictly exogenous and in addition $\mathbf{y}_{t}$ is independent of $\mathbf{x}_{\mathbf{1}}, \ldots, \mathbf{x}_{t-1}$ conditional on $\mathbf{x}_{\mathbf{t}}$ ' then we shall say that the relationship of $\mathbf{x}$ to $\mathbf{y}$ is static. 
Then our question is whether it is restrictive to assert that there exists a latent variable $\mathrm{c}$ such that the relationship of $\mathbf{x}$ to $\mathrm{y}$ is static conditional on c. We know that this is restrictive in the linear predictor case, since the weaker condition that $\boldsymbol{x}$ be strictly exogenous conditional on $c$ is restrictive. Unfortunately, there are no restrictions when we replace zero partial correlation by conditional independence. It follows that conditional strict exogeneity is restrictive only when combined with specific functional forms -a a truly nonparametric test cannot exist. Section 4 presents our framework for inference. Let $\underline{\mathbf{r}}_{\mathbf{i}}^{*}=$ $\left(1, \mathbf{x}_{\mathbf{i 1}}, \ldots . . \mathbf{x}_{\mathbf{i T}}, \mathbf{y}_{\mathbf{i l}}, \ldots . . \mathbf{y}_{\mathbf{i T}}\right)$ and assume that $\mathbf{r}_{\mathbf{i}}$ is independent and identically distributed (i.i.d.) for $i=1,2, \ldots$. Let $w_{1}$ be the vector formed from the squares and cross-products of the elements in $\mathbf{F}_{\mathbf{1}}$. Our framework is based on a simple observation: the matrix $\underset{\sim}{\boldsymbol{I}}$ of linear predictor coefficients is a function of $\mathbf{E}\left({\underset{\sim}{1}}_{\mathbf{1}}\right)$; if $\boldsymbol{I}_{\mathbf{1}}$ is i.i.d. then so is $\mathbb{w}_{\mathbf{i}}$; hence our problem is to make inferences about a function of a population mean under random sampling. This is straightforward and provides an asymptotic distribution theory for least squares that does not require a linear regression function or homoskedasticity. Stack the columns of $\underline{\pi}^{\prime}$ into a vector $\pi$ and let $\underline{\pi} \mathbf{z} \underline{\mathbf{h}}(\underline{\mu})$, where $\dot{y}=\mathbf{E}\left(\mathbf{w}_{\mathbf{1}}\right)$. Then the limiting distribution for least squares is normal with covariance matrix

$$
\Omega=\frac{\partial \underline{\sim}}{\partial \underline{\sim}}, V(\underset{\sim}{w}) \frac{\partial \underline{\sim}^{\prime}}{\partial \underline{\mu}} .
$$

We impose restrictions on $\mathbb{I}$ by using a minimum distance estimator. The restrictions can be expressed es $\underset{\sim}{\mu}=g(\theta)$, where $\theta$ is free to vary 
within some set $T$. Given the sample mean $\underset{\sim}{\mathfrak{w}}=\sum_{i=1}^{\mathbb{N}} \underset{\sim}{\mathbb{W}} / \mathbb{N}$, we choose $\hat{g}$ to minimize the distance between $\overline{\mathfrak{W}}$ and $\underset{\sim}{g}(\theta)$, using the following distance function:

$$
\underset{\sim}{\min }[\underset{\sim}{\bar{w}}-g(\theta)]^{+} \hat{V}^{-1}\left({\underset{\sim}{W}}_{i}\right)[\underset{\sim}{\bar{w}}-g(\theta)],
$$

where $\hat{V}\left({\underset{\sim}{i}}_{i}\right)$ is a consistent estimator of $V(\underset{\sim}{\mathbf{w}})$. This is a generalized least squares estimator for a multivariate regression model with nonlinear restrictions on the parameters; the only explanatory variable is a constant term. The limiting distribution of $\hat{\theta}$ is normal with covariance matrix $\left[\frac{\partial g_{g}^{\prime}}{\partial \underset{\sim}{\theta}} \nabla^{-1}\left(\underset{\sim}{w_{i}}\right) \frac{\partial g_{\tau}}{\partial g^{\prime}}\right]^{-1}$

An asymptotic distribution theory is also available when we use some matrix other than $\hat{V}^{-1}(\underset{\sim}{\mathbb{w}})$ in the distance function. This theory shows that $\hat{V}^{-1}\left({\underset{\sim}{i}}_{i}\right)$ is the optimal choice. However, by using suboptimal norms, we can place a number of commonly used estimators within this framework. The results on efficient estimation have some surprising consequences. The simplest example is a univariate linear predictor: $E^{*}\left(\mathbf{y}_{\mathbf{i}} \mid \mathbf{x}_{\mathbf{i 1}}, \mathbf{x}_{\mathbf{1 2}}\right)=$ $\pi_{0}+\pi_{1} \mathbf{x}_{\mathbf{i 1}}+\pi_{2} \mathbf{x}_{\mathbf{1 2}}$. Consider imposing the restriction that $\pi_{2} \mathbf{m} 0$; we do not want to maintain any other restrictions, such as linear regression, homoskedasticity, or normality. How shall we estimate $\pi_{1}$ ? Let $\hat{\pi}^{\prime}=\left(\hat{\pi}_{1}, \hat{\pi}_{2}\right)$ be the estimator obtained from the least squares regression of $y$ on $\mathbf{x}_{1}, \mathbf{x}_{2}$. We want to find a vector of the form $(\theta, 0)$ as close as possible to $\left(\hat{\pi}_{1}, \hat{\pi}_{2}\right)$, using $\hat{v}^{-1}(\hat{\mathbb{I}})$ in the distance function. since we are not using the conventional estimator of $\mathbf{v}(\underset{\sim}{\pi})$, the answer to this 
minimization problem is not, in general, to set $\hat{\theta}^{\wedge}=b_{y_{1}}$, the estimator obtained from the least squares regression of $y$ on $\mathbf{x}_{1}$. We can do better by using $b_{\mathbf{y x}_{1}}+\tau \hat{\pi}_{2}$; the asymptotic mean of $\pi_{2}^{\wedge}$ is zero if $\pi_{2}=0$, and if $b_{y_{1}}$ and $\hat{\pi}_{2}$ are correlated, then we can choose $\tau$ to reduce the asymptotic variance below that of ${ }^{b} \mathbf{y x}_{1}$.

This point has a direct counterpart in the estimation of simultaneous equations. The restrictions on the reduced form can be imposed using a mintmum distance estimator. This is more efficient than conventional estimators since it is using the optimal norm. In addition, there are generalizations of two- and three-stage least squares that achieve this efficiency gain at lower computational cost.

A related application is to the estimation of restricted ovariance matrices. Here the assumption to be relaxed is multivariate mo mality. We show that the conventional waximum likelihood estimator, wh ch assumes normality, is asymptotically equivalent to a minimum distance estimator. But that minfmum distance estimator is not. in general, using the optimal norm. Hence there is a feasible minimum distance estimator that is as least es good as the maximum likelihood estimator; it is strictly better in general for nonnormal distributions.

The minimum distance approach has an application to the multivariate probit model of Section 3. We begin by estimating $\mathbf{T}$ separate frobit specifications in which all leads and lags of $\mathbf{x}$ are included in the specification for each $y_{1 t}$ :

$$
P\left(y_{i t}=1 \mid x_{i I}, \ldots . . x_{i I}\right)=F\left(\pi_{t o}+\pi_{t I} x_{i I}+\ldots+\pi_{t T} x_{i I}\right),
$$


where $\bar{F}$ is the standard normal distribution function. Each of the $\mathrm{T}$ probit specifications is estimated using a maximum likelihood program for univariate probit analysis. There is some sacrifice of efficiency here, but it may be outweighed by the advantage of avoiding numerical integration. Given the estimator for $\underset{\sim}{\pi}$, we derive its asymptotic covariance matrix and then impose and test restrictions by using the minimum distance estimator.

Section 5 presents two empirical applications, which implement the, specifications discussed in Sections 2 and 3 using the inference procedures from section 4. The linear example is based on the panel of Young Men in the National Longitudinal Survey (Parnes); $y_{t}$ is the logarithm of the individual's hourly wage and $\mathbf{x}_{t}$ includes variables to indicate whether or not the individual's wage is set by collective bargaining; whether or not he lives in an SMSA; and whether or not he lives in the South. We present unrestricted least squares regressions of $\mathbf{y}_{t}$ on $\mathbf{x}_{\mathbf{l}}, \ldots .$. . xT, and we examine the form of the $\underset{\sim}{\mathbb{m}}$ matrix. There are significant leads and lags, but there is evidence in favor of a static relationship conditional on a latent variable; the leads and lags could be interpreted as just due to c, with $\mathbf{E}\left(\mathbf{y}_{t} \mid \mathbf{x}_{\mathbf{I}}, \ldots, \mathrm{xT}_{\mathbf{c}} \mathbf{c}\right)=\beta \mathbf{x}_{\mathbf{t}}+\mathrm{c}$. The estimates of $\beta$ that control for $\mathrm{C}$ are smaller in absolute value than the cross-section estimates. The union coefficient declines by 402, with somewhat larger declines for the SMSA and region coefficients. The second application presents estimates of a model of labor force participation. It is based on a sample of married women in the Michigan Panel Study of Income Dynamics. We focus on the relationship between 
participation and the presence of young children. The unrestricted $\underset{\sim}{I}$ matrix for the probit specification has significant leads and lags; but, unlike the wage example, there is evidence here that the leads and lags are not generated just by a latent variable. If we do impose this restriction, then the resulting estimator of $\beta$ indicates that the crosssection estimates overstate the negative effect of young children on the woman's participation probability.

The estimates for the logit functional form present some interesting contrasts to the probit results. The cross-section estimates, as usual, are in close agreement with the probit estimates. But when we use the conditional maximum likelihood estimator to control for $\mathrm{c}$, the effect of an additional young child on participation becomes substantially more negative than in the cross-section estimates; so the estimated sign of the bias is opposite to that of the probit results. Here the estimation method is having a first order effect on the results. There are a variety of possible explanations. It may be that the unrestricted distribution for $\mathrm{c}$ in the logit form is the key. Or, since there is evidence against the restriction that

$$
P\left(y_{1 t} \mid x_{i I}, \ldots . . x_{1 T}, c_{1}\right)=P\left(y_{i t} \mid x_{i t}, c_{i}\right)
$$

perhaps we are finding that imposing this restriction simply leads to different biases in the probit and logit estimates. 


\section{SPECIFICATION AND IDENTIFICATION: LINEAR MODELS}

\subsection{A Froduction Function Example}

We shall begin with a production function example, due to Mundlak (1961). ${ }^{2}$ Suppose that a farmer is producing a product with a Cobb-Douglas technology:

$$
y_{i t}=\beta x_{i t}+c_{i}+u_{i t} \quad(0<\beta<1 ; \quad i=1, \ldots . N t, \ldots . T)
$$

where $\mathbf{y}_{\mathbf{i t}}$ is the logarithm of output on the $\mathbf{t h}$ farm in season $t, \mathbf{x}_{\mathbf{i t}}$ is the logarithm of a variable input (labor), $\mathbf{c}_{\mathbf{i}}$ represents an input that is fixed over time (soil quality), and $\mathbf{u}_{\mathbf{i t}}$ represents a stochastic input (rainfall), which is not under the farmer's control. We shall assume that the farmer knows the product price (P) and the input price (W), which do not depend on his decisions, and that ha knows ci. The factor input decision, however, is made before knowing $\mathbf{u}_{\mathbf{i t}}$, and we shall assume that $\mathbf{x}_{\mathbf{1 t}}$ is chosen to maximize expected profits. Then the factor demand equation is

$$
x_{t}=\left\{\ln \beta+\ln \left[E\left(e^{u} \mid J_{t}\right)\right]+\ln \left(P_{t} / w_{t}\right)+c\right\} /(1-\beta)
$$

where $J_{t}$ is the information set available to the farmer when he chooses $\mathbf{x}_{\boldsymbol{t}}$, and we have suppressed the $i$ subscript.

$$
\text { Assume first that } \mathbf{u}_{t} \text { is independent of } \mathrm{Jt} \text {, so that the farmer cannot }
$$

do better than using the unconditional mean. In that case we have

$$
E\left(y_{t} \mid x_{1}, \ldots, x_{T}, c\right)=\beta x_{t}+c
$$


So if $c$ is observed, only one period of data is needed: the least squares regression of $Y_{1}$ on $x_{1}$, C provides a consistent estimator of $\beta$ as $N+\infty$. Now suppose that $\mathrm{c}$ is not observed by the econometrician, although it is known to the farmer. Consider the least squares regression of $\mathbf{y}_{1}$ on $\mathbf{x}_{1}$, using just a single cross-section of the data. The population counterpart is

$$
E *\left(y_{1} \mid x_{1}\right)=\pi_{0}+\pi x_{1}
$$

where $\mathbf{E}^{*}$ is the minimum mean-square error linear predictor (the wide-sense regression function):

$$
\pi=\operatorname{Cov}\left(y_{1}, x_{1}\right) / V\left(x_{1}\right), \pi_{0}=E\left(y_{1}\right)-\pi E\left(x_{1}\right)
$$

We see from (2.1) that $c$ and $x_{1}$ are correlated; hence $\pi \neq \beta$ and the least squares estimator of $\boldsymbol{B}$ does not converge to $\boldsymbol{B}$ as $\mathbf{N} \rightarrow \infty$. Furthermore, with a single cross section, there may be no internal evidence of this omittedvariable bias.

Now the panel can help to solve this problem. Mundlak's solution was to include farm specific indicator variables: a least squares regression of $y_{i t}$ on $x_{i t}, d_{1 t}(1=1, \ldots, \mathbb{N} ; t-1, \ldots . . T)$, where $\mathbb{d}_{-1 t}$ is a $\mathbb{N} \mathbb{I}$ vector of zeros except for a one in the $i \underline{\text { th }}$ position. So this solution treats the $c_{i}$ as a set of parameters to be estimated. It is a "fixed effects" solution, which we shall contrast with "random effects." The distinction is that under a fixed effects approach, we condition on the $\boldsymbol{c}_{\mathfrak{i}}$, so that their distribution plays no role. A random effects approach invokes a distribution for $c$. In a Bayesian framework, $\beta$ and the $c_{1}$ would be treated symmetrically, with a prion distribution for both. Since I am only going to 
use asymptotic results on inference, however, a "gentle" prior distribution for $\beta$ will be dominated. That this need not be true for the $c_{i}$ is one of the interesting aspects of our problem.

We shall do asymptotic inference as $\mathrm{N}$ tends to infinity for fixed $\mathrm{T}$. Since the number of parameters $\left(c_{i}\right)$ is increasing with sample size, there is a potential "incidertal parameters" problem in the fixed effects approach. This does not, however, pose a deep problem in our example. The least squares regression with the indicator variables is algebraically equivalent to the least squares regression of $\mathbf{y}_{\mathbf{i t}}-\overline{\mathbf{y}}_{\mathbf{1}}$ on $\mathbf{x}_{\mathbf{i t}}-\overline{\mathbf{x}}_{\mathbf{i}}(\mathbf{i}=\mathbf{1}, \ldots . . \mathrm{N}$; $\mathrm{t}=1$, . . . T), where $\overline{\mathrm{y}}_{\mathbf{i}}=\sum_{\mathrm{t}=1}^{\mathrm{T}} \mathrm{y}_{\mathbf{i t}} / \mathrm{T}, \overline{\mathrm{x}}_{\mathbf{i}}=\Sigma_{\mathrm{t}=1}^{\mathrm{T}} \mathbf{x}_{\mathbf{1 t}} / \mathrm{T}$. If $\mathrm{T}=2$, this reduces to a least squares regression of $y_{i 2}-y_{i 1}$ on $x_{i 2}-x_{i 1}$. Since

$$
E\left(y_{i 2}-y_{i 1} \mid x,-x_{i 1}\right)=\beta\left(x_{i 2}-x_{i 1}\right),
$$

the least squares regr ssion will provide a consistent stimator of $\beta$ if there is sufficient variation in $x_{12}-x_{11} \cdot 3$

\subsection{Fized Effects and Incidental Parameters}

The incidental parameters can create real difficulties. Suppose that $\mathbf{u}_{\mathbf{i t}}$ is independently and identically distributed (i.i.d.) across farms and periods with $\mathbf{v}\left(\mathbf{u}_{\mathbf{i t}}\right)=\mathbf{z}^{2}$. Then under a normality assuretion, the maximum likelihood estimator of $\sigma^{2}$ converges (almost surely) to $\sigma^{2}(\mathrm{~T}-1) / \mathrm{T}$ as $\mathrm{N}+\infty$ with $\mathrm{T}$ fixed. 4 ine failure to correct for degrees of freedom leads to a serious inconsistency when $I$ is small. For another example, consider the following autoregression: 


$$
\begin{aligned}
& y_{i 1}=a y_{i 0}+c_{i}+u_{i 1}, \\
& y_{i 2}=\beta y_{i 1}+c_{i}+u_{i 2} .
\end{aligned}
$$

Assume that $\mathbf{u}_{\mathbf{i} 1}$ and $\mathbf{u}_{\mathbf{i} 2}$ are i.i.d. conditional on $\mathbf{y}_{10}$ and $\mathrm{ci}$, and that they follow a normal distribution $\left(\mathrm{N}\left(\mathrm{O}, \sigma^{2}\right)\right.$ ). Consider the likelihood function corresponding to the distribution of $\left(y_{i 1}, y_{i 2}\right)$ conditional on $y_{i o}$ and $c_{i}$. The $\log _{\text {-likelihood function is quadratic } i \bar{n}} \beta, \mathrm{cl}, \ldots . . c_{\mathbb{N}}$ (given $\sigma^{\mathcal{S}}$ ), and the maximum likelihood estimator of $B$ is obtained from the least squares regression of $y_{12}-y_{11}$ on $y_{i 1}-y_{10}\left(i-1_{1} \ldots . . N\right)$. Since $\mathbf{u}_{\mathbf{i l}}$ is correlated with $\mathrm{y}_{11}$, and

$$
y_{i 2}-y_{i 1}=\beta\left(y_{i 1}-y_{i 0}\right)+u_{i 2} \cdot u_{i 1},
$$

it is clear that

$$
E\left(y_{12}-y_{11} \mid y_{i 1}-y_{i 0}\right) \neq \beta\left(y_{i 1} \quad y_{i 0}\right)
$$

and the maximum likelihood estimator of $\beta$ is not conoistent. If the distribution of yio conditional on $\boldsymbol{c}_{\boldsymbol{i}}$ does not depend on $\boldsymbol{B}$ or $\boldsymbol{c}_{\boldsymbol{i}}$, then the likelihood function based on the distribution of $\left(y_{i 0}, y i l, y_{i 2}\right)$ conditional on $\boldsymbol{c}_{\boldsymbol{1}}$ gives the same inconsistent maximum likelihood estimator of B. If the distribution of (yio, $y_{\mathbf{i 1}}, \mathbf{y}_{\mathbf{1 2}}$ ) is stationary, then the estimator obtained from the least squares regression of $y_{i 2}=y_{i 1}$ on $y_{11}-y_{i 0}$ converges, as $\mathbf{N}+\infty$, to $(\beta-1) / 2 .^{5}$

\subsection{Rondom Effects and Specification Analysis}

We have seen that the success of the fixed effects estimator in the production function example must be viewed with some caution. The inci- 
dental parameter problem will be even more serious when we consider nonlinear models. So we shall consider next a random effects treatment of the production function example; this will also provide a convenient framework for specification analysis. 6

Assume that there is some joint distribution for $\left(\mathbf{x}_{\mathbf{i l}}, \ldots . . \mathbf{x}_{\mathbf{i T}}, \mathbf{c}_{\mathbf{i}}\right)$, which does not depend upon $i$, and consider the regression function that does not condition on $\mathrm{c}$ :

$$
E\left(y_{i t} \mid x_{11}, \ldots, x_{i T}\right)=\beta x_{1 t}+E\left(c_{i} \mid x_{11}, \ldots, x_{i T}\right)
$$

The regression function for $\mathbf{c}_{\mathbf{1}}$ given $\mathbf{x} \cdot \mathbf{z} \mathbf{\mathbf { i }}\left(\mathbf{x}_{\mathbf{1 1}}, \ldots, \cdot \mathbf{x}_{\mathbf{i} \mathbf{T}}\right)$ will generally be some nonlinear function. But we can specify a minimum mean-square error linear predictor:'

$$
E *\left(c_{i} \mid x_{i 1}, \ldots . . x_{1 T}\right)=\psi+\lambda_{1} x_{i I}+\ldots+\lambda_{T} x_{i T}=\psi+\lambda_{\sim}^{\prime} x_{i},
$$

where $\lambda=\mathbf{v}^{-1}({\underset{\mathbf{x}}{1} \mathbf{1}}) \operatorname{Cov}\left(\mathbf{x}_{\mathbf{i}}, \mathbf{c}_{\mathbf{I}}\right)$. No restrictions are being imposed here (2.2) is simply giving our notation for the linear predictor.

Now we have

$$
E *\left(y_{1 t} \mid{\underset{\sim}{x}}_{i}\right)=\psi+\beta \mathbf{x}_{1 t}+\lambda_{\sim}^{\top} \mathbf{x}_{i}
$$

Combining these linear predictors for the $\mathbf{T}$ periods gives the following multivariate linear predictor: ${ }^{8}$

$$
\begin{aligned}
& E^{*}\left(\underline{Y}_{i} \mid \mathbf{x}_{1}\right)=\prod_{\sim 0}+\underset{\sim}{x_{1}} \\
& I I=\operatorname{Cov}\left(y_{1}, x_{i}^{\prime}\right) v^{-1}\left(\underline{x}_{1}\right)=\beta I+\mathcal{I} \lambda^{\prime},
\end{aligned}
$$


where $y_{i}^{\prime}=\left(y_{i 1}, \ldots, y_{i: T}\right), I$ is the $T \times T$ identity matrix, and $l$ is a $T \times 1$ vector of ones.

The $\underset{\sim}{\dddot{m}}$ matrix is a useful tool for analyzing this model. Consider first the estimation of 6 : if $T=2$ we have

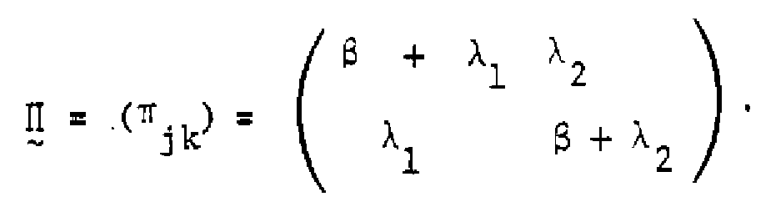

Hence

$$
\beta=\pi_{11}-\pi_{21}=\pi_{22}-\pi_{12} *
$$

So given a consistent estimator for $\underset{\sim}{\boldsymbol{m}}$, we can obtain a consistent estimator for 5. The estlmation of $\underset{\sim}{\mathbb{I}}$ is almost a standard problem in multivariate regression; but, due to the nonlinearity in $\mathbf{E}\left(\mathbf{c}_{\mathbf{1}} \mid \mathbf{x}_{\mathbf{i}}\right)$, we are estimating only a wide-sense regression function, and some care is needed. It turns out that there is a way of looking at the problem which allows a straightforward treatment, under very weak assumptions. We shall develop this in the section on inference.

We see in (2.3) that there are restrictions on the $\underset{\sim}{\pi}$ matrix. The offdiagonal elements within the same column of $\underset{\Xi}{\approx}$ are all equal. The $\mathrm{T}^{z}$ elements of $\underline{I}$ are functions of the $\mathrm{T}+1$ parameters $\beta, \lambda_{1}, \ldots . . \lambda_{\mathbf{T}}$. This suggests an obvious specification test. Or, backing up a bit, we could begin with the specification that $\mathbb{I}=\boldsymbol{B} \boldsymbol{I}$. Then passing to (2.3) would be a test for whether there is a time-invariant omitted variable that is correlated with the $\mathbf{x}^{\prime} \mathbf{s}$. The test of $\mathbb{I}=\boldsymbol{\beta} \underset{\sim}{\boldsymbol{l}}+\underset{\sim}{\boldsymbol{\lambda}} \boldsymbol{\sim}$ against an unrestricted $\underset{\sim}{\boldsymbol{\pi}}$ would be an omibus test of a variety of misspecifications, some of which will be considered next. ${ }^{9}$ 
18

Suppose that there is serial correlation in $\mathbf{u}_{\mathbf{w}}$ with $\mathbf{u}_{\mathbf{t}} \mathbf{=} \rho_{\mathbf{u}} \mathbf{t}_{\mathbf{1}}+\mathbf{w}_{\mathbf{t}}$, where $w_{t}$ is independent of $I_{t}$ and we have suppressed the $i$ subscripts.

Now we have

$$
E\left(e^{u_{t}} \mid J_{t}\right)=e^{p u_{t-1}} E\left(e^{w}\right)
$$

So the factor demand equation becomes

$$
x_{t}=\left\{\ln \beta+\ln \left[E\left(e^{" t}\right)\right]+\ln \left(P_{t} / w_{t}\right)+\rho_{u}-1+c\right\} /(1-\beta)
$$

Suppose that there is no variation in prices across the farms, so that the $\mathbf{P}_{\mathbf{t}} / \boldsymbol{W}_{\mathbf{t}}$ term is captured in period specific intercepts, which we shall suppress.

Then we have

$$
E^{*}\left(y_{t} \mid x_{1}, \ldots x_{T}\right)=B x_{t}+\left(1-p^{-1}\right)\left(\lambda_{1} x_{1}+\ldots+\lambda_{T} x_{T}\right)+\varphi x_{t+1},
$$

where $\varphi=\rho^{-1}(1-\beta)$.

So the $\underset{\sim}{\mathbb{m}}$ matrix would indicate a distributed lead, even after controlling for $c$. If instead there is a first order moving average, $\mathbf{u}_{\mathbf{t}}=\mathbf{w}_{\mathbf{t}}+\mathrm{pw}_{\mathbf{t}-\mathbf{I}}$, then

$$
E\left(e^{u_{t}} \mid J_{t}\right)=e^{P_{W} t-I} E\left(e^{{ }^{W}}\right),
$$

and a bit of algebra gives 


$$
\left.\operatorname{E(y_{t}} \mid x_{1}, \ldots x_{T}\right)=x_{t}-p^{-1}\left(\lambda_{1} x_{1}+\ldots .+\lambda_{T} x_{T}\right)+\varphi x_{t+1} .
$$

Once again there is a distributed lead, but now $\beta$ is not identified from the II matrix.

2.4. A Corwumer Demand Exomple

2.4.a. Certainty

We shall follow Ghez and Becker (1975), Heckman and MaCurdy (1980), and Macurdy (198I) in presenting a life-cycle model under certainty. suppose that the consumer is maximizing

$$
V=\sum_{t-1}^{\tau} \rho^{(t-1)} U_{t}\left(C_{t}\right)
$$

subject to

$$
\sum_{t=:}^{\tau} Y^{-(t-1)} P_{t} C_{t} \leq B, C_{t} \geq 0(t=1, \ldots . . \tau)
$$

where $\rho^{-1}-1$ is the rate of time preference, $\mathrm{Y}-1$ is the (nominal) interest rate, $\mathbf{c}_{\mathbf{t}}$ is consumption in period $t_{\boldsymbol{r}} \mathbf{P}_{\boldsymbol{t}}$ is the price of the consumption good in period $t$, and $B$ is the present value in the initial period of lifetime income. In this certainty model, the consumer faces a single lifetime budget constraint.

If the optimal consumption is positive in every period, then

$$
\left.U_{t}^{\prime} ; C_{t}\right)=(Y \rho)^{-(t-1)}\left(P_{t} / P_{1}\right) U_{1}^{\prime}\left(C_{1}\right)
$$

A convenient functional form is $\mathrm{J}_{t}(C)=A_{t} C^{\delta} / \delta\left(A_{t}>0, \delta<1\right)$; then we have $(2.4)$

$$
y_{t}=\beta x_{t}+\varphi(t-1)+c+u_{t},
$$


where $y t=\ln C t, x_{t}=\ln P t, c=(\delta-1)^{-1} \ln \left[U_{1}^{\prime}\left(C_{1}\right) / P_{1}\right]$,

$u_{t}=(1-\delta)^{-1} \ln A t, \beta=(\delta-1)^{-1}$, and $\varphi=(1-\delta)^{-1} \ln (\gamma \rho)$. Note that $c$ is determined by the marginal utility of initial wealth: $\mathrm{U}_{1}^{\prime}\left(\mathrm{C}_{1}\right) / \mathrm{P}_{1}=\partial \mathrm{V} / \partial \mathbf{B}$.

We shall assume that At is not observed by the econometrician, and that it is independent of the P's. Then the nodel is similar to the production function example if there is price variation across consumers as well as over time. There will generally be correlation between $c$ and $\left(\mathbf{x}_{1}, \ldots . . \mathbf{x}_{\mathrm{T}}\right)$. As before we have the prediction that $\underset{\sim}{\mathbb{N}}=\beta I+\underset{\sim}{\ell} \lambda^{\prime}$, which is testable. A consistent estimator of $\beta$ can be obtained with only two periods of data since

$$
y_{t}-y_{t-1}=\beta\left(x_{t}-x_{t-1}\right)+\varphi+u_{t}-u_{t-1} .
$$

We shall see next how these results are affected when we allow for some uncertainty.

\section{4.b. Uncertainty}

We shall present a highly simplified model in order to obtain some explicit results in the uncertainty case. The consumer is maximizing

$$
E\left[\sum_{t=1}^{T} P{ }^{t-1_{U_{t}}}\left(c_{t}\right) 1\right.
$$

subject to

$$
\begin{aligned}
& P_{1} C_{1}+S_{1} \leq B, \\
& P_{t} C_{t}+S_{t} \leq Y s_{t-1}, C_{t} \geq 0, s_{t} \geq 0(t=1, \ldots . \tau) .
\end{aligned}
$$


The only source of uncertainty is the future prices. The consumer is allowed to borrow against his future income, which has a present value of $B$ in the initial period. The consumption plan must have $C_{t}$ a function only of information available at date $t$.

It is convenient to set $\boldsymbol{\tau}=\boldsymbol{\infty}$ and to assume that $\mathbf{P}_{\mathbf{t}+\mathbf{1}} / \mathbf{P}_{\mathbf{t}}$ is i.i.d. $(t=1,2, \ldots)$. If $U_{t}(c)=A_{t} c^{\delta} / \delta$, then we have the following optimal plan: 10

$$
\begin{aligned}
& c_{1}=d_{1} B / P_{1}, s_{1}=\left(1-d_{1}\right) B \\
& c_{t}=d_{t} \gamma s_{t-1} / P_{t}, s_{t}=\left(1-d_{t}\right) \gamma s_{t-1} \quad(t-2,3, \ldots) .
\end{aligned}
$$

where

$$
\begin{aligned}
& d_{t}=\left[1+E_{t+1}+\left(E_{t+1} E_{t+2}\right)+\ldots\right]^{-1}, \\
& f_{t}=\left(\rho K A_{t} / A_{t-1}\right)^{[I /(1-\delta)]}, \quad k=\gamma^{\delta} E\left[\left(P_{t-1} / P_{t}\right)^{\delta}\right] .
\end{aligned}
$$

It follows that

$$
y_{t}-y_{t-1}=(-1)\left(x_{t}-x_{t-1}\right)+5+u_{t}-u_{t-1},
$$

where $\mathbf{y}, \mathbf{x}, \mathbf{u}$ are defined as in $(2.4)$ and $\zeta=(1-\delta)^{-1} \ln (\mathrm{PK})+\ln \gamma$.

We see that, in this particular example, the appropriate interpretation of the change regression is very sensitive to the amount of information available to the consumer. In the uncertainty case, a regression of $\left(\ln C_{\mathbf{t}}=\right.$ en $\left.C_{t-1}\right)$ on $\left(\boldsymbol{l n} \boldsymbol{P}_{\mathbf{t}}=\right.$ en $\left.\boldsymbol{P}_{\mathbf{t}-1}\right)$ does not provide a cor:sistent estimator of $(\delta-1)^{-1}$; in fact, the estimator converges to -1 , with the implied estimator of $\delta$ converging to 0 . 


\section{2.a.c. Labor Supply}

We shall consider a certainty model in which the consumer is maxi-

mizing

(2.6) $\quad v \quad \sum_{t=1}^{\tau} \rho^{(t-1)} U_{t}\left(C_{t}, L_{t}\right)$

subject to

$$
\begin{aligned}
& \sum_{t=1}^{\tau} \gamma^{-(t-1)}\left(P_{t} C_{t}+W_{t} L_{t}\right) \leq B+\sum_{t-1}^{\tau} \gamma^{-(t-1)} W_{t} \vec{L}, \\
& c_{t} \geq 0,0 \leq L_{t} \leq \bar{L} \quad(t=1, \ldots . \tau),
\end{aligned}
$$

where $I_{t}$ is leisure, $W_{t}$ is the wage rate, $B$ is the present value in the initial period of nonlabor income, and $\overline{\mathrm{L}}$ is the time endowment. We shall assume that the inequality constraints on $L$ are not binding; the participation decision will be discussed in tbe section on nonlinear models. If $\boldsymbol{U}_{\mathbf{t}}$ is additively separable,

$$
U_{t}(C, L)=U_{t}^{*}(C)+\tilde{U}_{t}(L)
$$

and if $\tilde{\mathrm{t}}_{\mathbf{t}}(\mathrm{L})=\mathrm{A}_{\mathbf{t}} \mathrm{L}^{\delta} / \delta$, then we have

$$
y_{t}=\beta x_{t}+\varphi(t-1)+c+u_{t}
$$

where $y_{t}=\ln L_{t}, x_{t}=\ln \mathrm{w}_{t}, c=(\delta-1)^{-1} \ln \left[\tilde{U}_{1}^{*}\left(\mathrm{~L}_{1}\right) / \mathrm{w}_{1}\right]$,

$u_{t}=(1-\delta)^{-1} \ln A_{t}, \beta=(\delta-1)^{-1}$, and $\varphi=(1-6)^{-1} \ln (\gamma \rho)$. Once again $c$ is determined by the marginal utility of initial wealth: $\tilde{U}_{1}^{\prime}\left(L_{1}\right) / w_{1}=\partial \mathrm{V} / \partial \mathrm{B}$.

We shall assume that $\mathbf{A}_{\boldsymbol{t}}$ is not observed by the econometrician. There will generally be a correlation between $c$ and $\left(\mathbf{x}_{1}, \ldots . . \mathbf{x}_{\mathbf{T}}\right)$, since $\mathrm{L}_{\mathbf{1}}$ depends 
hypothesis in (2.8) implies that if $T \geq 4$, there are $(T-3)(T-2) / 2$ overidentifying restrictions.

Consider next a Granger definition of "y does not cause $\mathbf{x}$ conditional on $c^{\prime \prime}$ :

$(2.10)$

$$
\begin{array}{r}
E^{*}\left(x_{t+1} \mid x_{1}, \ldots . x_{t}, y_{I}, \ldots, y_{t}, c\right)=E^{*}\left(x_{t+1} \mid x_{1}, \ldots . x_{t}, c\right) \\
(t=1, \ldots . . T-1) .
\end{array}
$$

Define the following linear predictors:

$$
\begin{aligned}
& x_{t+1}=\psi_{t 1} x_{1}+\ldots+\psi_{t t} x_{t}+\varphi_{t 1} y_{I}+\ldots+\varphi_{t t} y_{t}+\zeta_{t+1} c+v{ }_{t+1}, \\
& E *\left(v_{t+1} \mid x_{1}, \ldots, x_{t}, y_{I}, \ldots . . y_{t}, c\right)=0 \quad(t=1, \ldots . .1-1) .
\end{aligned}
$$

Then $(2.10)$ is equivalent to $\varphi_{\mathbf{t s}}=0$. We can rewrite the system, imposing $\varphi_{\text {ts }}=0$, as follows:

$$
\begin{aligned}
& x_{t+1}=\tilde{\psi}_{t I} x_{1}+\ldots+\dot{\psi}_{t, t-1} x_{t-1}+\tau_{t} x_{t}+v_{t+1}, \\
& \tilde{\psi}_{t s}=\psi_{t s}-\left(\zeta_{t+1} / \zeta_{t}\right) \psi_{t-1, s}, \tau_{t}=\psi_{t t}+\left(\zeta_{t+1} / \zeta_{t}\right), \\
& v_{t+1}=v_{t+1}-\left(\zeta_{t+1} / \zeta_{t}\right) v_{t}, E\left(x_{s} \tilde{v}_{t+1}\right)=E\left(y_{s} \tilde{v}_{t+1}\right)=0 \\
& (s<t-1 ; t-2, \ldots . I-1) .
\end{aligned}
$$

In the equation for $\mathbf{x}_{t+1}$, there are $t$ unknown parameters, $\psi_{t}, \ldots . . \psi_{t, t-1}$ ' ' $t$ ' and $\mathbf{2 ( t - 1 )}$ orthogonality conditions. Hence there are $t-2$ restrictions

$$
(3 \leq t \leq T * 1)
$$

It follows that the Granger condition for "y does not cause $\mathbf{x}$ conditional on $\mathbf{c}^{\prime \prime}$ implies $(\mathbf{T}-\mathbf{3})(\mathbf{T}-\mathbf{2}) / 2$ restrictions, which is the same number of restrictions implied by the sims condition. In fact, it is a consequence of sims" (1972) theorem, as extended by Hosoya (1977), that the two sets of 
restrictions are equivalent; this is not immediately obvious from a direct comparison of $(2.9)$ and (2.19).

In terms of tie .? matrix, conditional strict exogeneity implies that

$$
\begin{aligned}
& \underline{\sim}=\underline{\sim}+\underline{\sim} \underline{\sim}^{\prime},
\end{aligned}
$$

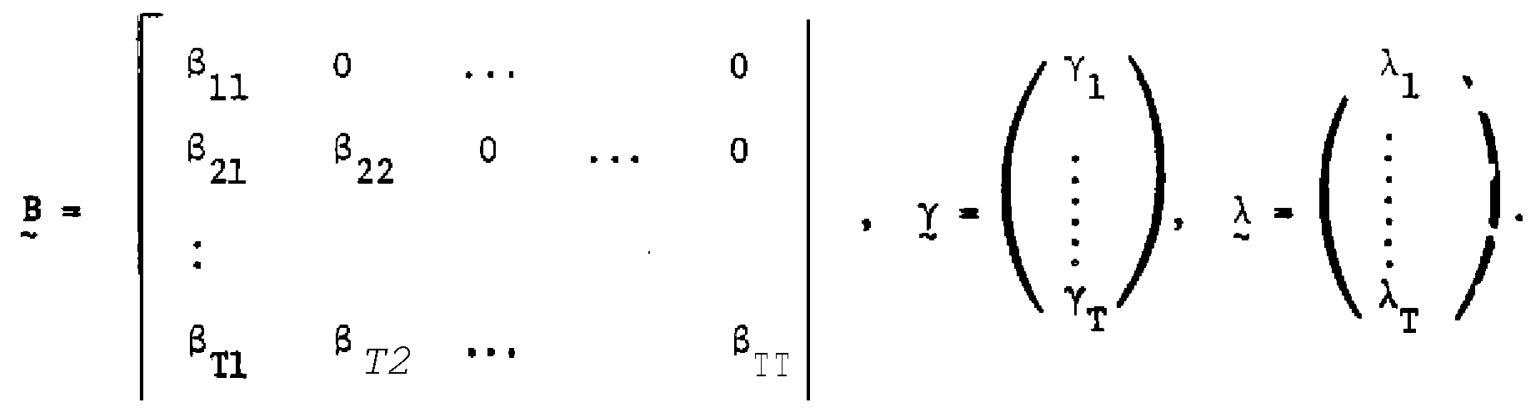

These nonlinear restrictions can be imposed and tested using the minimum

distance estimator to be developed in the inference section. Alternatively, we can use the transformations in (2.9) or in (2.11). These transfornations give us "simul taneous equations" systems with linear restrictions; (2.9) can be estimated using three-stage least squares. A generalization of three-stage least squares, which does not require homoskedasticity assumptions, is developed in the inference section. It is asymptotically equivalent to imposing the nonlfnear restrictions directly on $\underset{\sim}{\mathbb{\pi}}$, using the minimum distance estimator.

\subsection{Lagged Dependent Variables}

For a specific example, write the labor supply model in (2.7)

as follows:

$$
\begin{aligned}
& y_{t}=\delta_{1} x_{t}+\delta_{2} x_{t-1}+\delta_{3} y_{t-1}+v_{t^{\prime}} \\
& E^{*}\left(v_{t} \mid x_{1}, \ldots, x_{T}\right)=0 \quad(t=1, \ldots T) ;
\end{aligned}
$$

this reduces to (2.7) if $\delta_{2}=-\delta_{1}$ and $\delta_{3}=1$. If we assume that $\mathbf{v}_{\mathbf{t}}=\mathbf{w}+\mathbf{e}_{\mathbf{t}}$, 
upon wages in all periods. If At is independent of the $w^{\prime} s$, then we have the prediction that $\prod_{\sim}=\beta I+\ell \lambda^{\prime}$. If, however, wages are partly determined by the quantity of previous work experience, then there will be lags and leads in addition to those generated by $c$, and $\underset{\sim}{\pi}$ will not have this simple structuret.

It would be useful at this point to extend the uncertainty model to incorporate uncertainty about future wages. Unfortunately, a comparably simple explicit solution is not available. But we may conjecture that the correct interpretation of a regression of $\left(\ln \mathrm{L}_{\mathbf{t}}-\ln \mathrm{L}_{\mathrm{t}-1}\right)$ on $\left(\ln \mathrm{w}_{t}-\ln \mathrm{w}_{t-1}\right)$ is also sensitive to the amount of Information available to the consumer.

\subsection{Strict Exogeneity Londitional on a Latent Variable}

We shall relate the specification analysis of $\mathbb{\pi}$ to the causality definitions of Granger (1969) and Sims (1972). Consider a sample in which $t=1$ is the first period of the individual's (economic) life. 12 A Sims definition of " $\mathbf{x}$ is strictly exogenous" is

$$
E \star\left(y_{t} \mid x_{1}, x_{2}, \ldots\right)=E^{*}\left(y_{t} \mid x_{1}, \ldots . . x_{t}\right) \quad(t=1,2, \ldots)
$$

In this case $\mathbb{I}$ is lower triangular: he elements above the main diagonal are all zero. This fails to hold in the models we have been considering, due to the omitted variable c. But, in some cases, we do have the following property:

$$
E^{\star}\left(y_{t} \mid x_{1}, x_{2}, \ldots, c\right)=E^{\star}\left(y_{t} \mid x_{1}, \ldots>x_{t}, c\right)(t=1,2, \ldots)
$$

It was stressed by Granger (1969) that the assessment of noncausality depends crucially on what other variables are being conditioned on. The 
novel feature of $(2.8)$ is that we are asking whether there exists some latent variable (c) such that $\mathbf{x}$ is strictly exogenous conditional on $\mathrm{c}$. The question is not vacuous since $\boldsymbol{c}$ is restricted to be time invariant. Let us examine what restrictions are implied by (2.8). Define the following linear predictors: 13

$$
\begin{aligned}
& y_{t}=\beta_{t l} x_{1}+\ldots+\beta_{t T} x_{T}+\gamma_{t} c+u_{t}, \\
& E *\left(u_{t} \mid x_{1}, \ldots, x_{T}, c\right)=0 \quad(t=1, \ldots, T) .
\end{aligned}
$$

Then (2.8) is equivalent to $\boldsymbol{\beta}_{\text {ts }}=0$ for $s>t$. If $\gamma_{1} \neq 0$, we can choose a scale normalization for $\mathbf{c}$ such that $\boldsymbol{\gamma}_{1}=1$. Then we can rewrite the system with $\beta_{\text {ts }}=0(s>t)$ as follows:

$$
\begin{aligned}
& y_{t}=\tilde{\beta}_{t 1} x_{1}+\beta_{t 2} x_{2}+\ldots .+\beta_{t t} x_{t}+\gamma_{t} y_{1}+\tilde{u}_{t}, \\
& \tilde{\beta}_{t 1}=\beta_{t I}=\gamma_{t} \beta_{I 1}, \tilde{u}_{t}=u_{t}-\gamma_{t} u_{1}, \\
& E\left(x_{s} u_{t}\right)=0(s-1, \ldots . . T ; t=2, \ldots . . T)
\end{aligned}
$$

Consider the "instrumental variable" orthogonality conditions implied by $\mathbf{E}\left(\mathbf{x}_{\mathbf{s}} \tilde{\mathbf{u}}_{\mathbf{t}}\right)=0$. In the $\mathbf{y}_{\mathbf{T}}$ equation, we have $\mathbf{T}+\mathbf{1}$ unknown coefficients: $\tilde{\beta}_{T 1}, \beta_{T 2}, \ldots, 8, . \gamma_{T}$, and $T$ orthogonality conditions. So these coefficients are not identified. In the $\mathbf{y}_{\mathbf{I}-\mathbf{1}}$ equation, however, we have just enough orthogonality conditions; and in the $\mathrm{y}_{\mathbf{T}-\mathbf{j}}$ equation ( $j \leq \mathrm{I}-\mathrm{Z}$ ), we have $j-1$ more than we need since there are $\mathbf{T}-\mathbf{j}+1$ unknown coefficients:

$\tilde{\beta}_{T-j, 1}, \beta_{T-j, 2}, \ldots, B_{T-j}, \mathbf{T}-\mathbf{j}, \gamma_{T-j}$, and $T$ orthogonality conditions: $\mathbf{E}\left(\mathbf{x}_{\mathbf{s}} \tilde{u}_{\mathbf{T}-j}\right)=0(\mathbf{s}=1, \ldots . \mathrm{T})$. It follows that, subject to a rank condition, we can identify $\beta_{\mathbf{t s}}, \boldsymbol{\gamma}_{\mathbf{t}}$, and $\tilde{\beta}_{\mathbf{t l}}$ for $2 \leq s \leq t \leq \mathbf{T}-1$. In addition the 
where $w$ is uncorrelated with the $x$ 's and $e_{t}$ is i.i.d. and uncorrelated with the $x$ 's and $w$, then we have the autoregressive, variance-components model of Balestra and Nerlove (1966) .14 In keeping with our general approach, we shall avoid placing restrictions on the serial correlation structure of $v_{t}$, our inference procedures will be based $0: 1$ the strict exogeneity condition that $E *\left(v_{t} \mid x_{1}, \ldots . x_{T}\right)=0$.

We can fit this model into the $\stackrel{\Pi}{I}$ matrix framework by using recursive substitution to obtain the reduced form:

$$
\begin{aligned}
& y_{t}=\beta_{t I} x_{1}+\ldots+\beta_{t t} x_{t}+\gamma_{t} c+u_{t}, \\
& E *\left(u_{t} \mid x_{1}, \ldots, x_{T}\right)=0,
\end{aligned}
$$

where

$$
\begin{array}{r}
\beta_{t s}=\left(\delta_{2}+\delta_{3} \delta_{1}\right) \delta_{3}^{t-s-1}, \beta_{t t}=\delta_{1}, \gamma_{t}=\delta_{3}^{t-1}, \\
c=\delta_{2} x_{0}+\delta_{3} y_{0}, v_{t}=v_{t}+\delta_{3} v_{t-1}+\ldots . \delta_{3}^{t-1} v_{1} \\
(1 \leq s \leq t-1 . t=1, \ldots . T) .
\end{array}
$$

(We are assuming that (2.12) holds for $t \geq 1$, but data on $\left(x_{0}, y_{0}\right)$ are not available.)

Hence this model satisfies the cond..tional strict exogeneity restrictions,

$$
\underline{\Pi}=\underline{B}+\underline{Y} \underline{\lambda}^{\prime}
$$

where $\mathrm{B}$ is lower triangular. The $Y^{\prime}$ term is generated by the projection of the initial condition $\left(\delta_{2} x_{0}+\delta_{3} y_{0}\right)$ on $x l, \ldots . x_{T^{*}}{ }^{15}$

Estimation can proceed by using the minimum distance procedure to impose

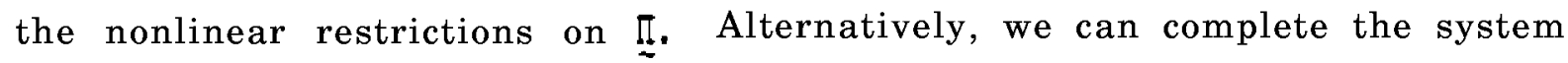


in (2.12) with

$$
\mathrm{y}_{1}=\varphi_{1} \mathrm{x}_{1}+\ldots+\varphi_{\mathrm{T}} \mathrm{x}_{\mathrm{T}}+\mathrm{v}_{1}
$$

this is just notation for the identity

$$
y_{1}=E^{*}\left(y_{1} \mid x_{1}, \ldots . x_{T}\right)+\left[y_{1}-E^{*}\left(y_{1} \mid x_{1}, \ldots, x_{T}\right)\right]
$$

Then we can apply the generalized three-stage least squares estimator to be developed in the inference section. It achieves the same limiting distribution at lower computational coat, since the restrictions in this form are linear and can be imposed without requiring iterative optimization techniques.

Now consider a $\leq$ scond order autoregression:

$$
\begin{aligned}
& y_{t}=\delta_{1} x_{t}+\delta_{2} x_{t-1}+\delta_{3} y_{t-1}+\delta_{4} y_{t-2}+t^{\prime} \\
& E *\left(v_{t} \mid x_{1}, \ldots . . x_{T}\right)=0 \quad(t=1, \ldots T) .
\end{aligned}
$$

Recursive substitution gives

$$
\begin{aligned}
& y_{t}=\beta_{t 1} x_{1}+\cdots+\beta_{t t} x_{t}+\gamma_{t 1} C_{1}+\gamma_{t 22} c+y_{t} \\
& E *\left(u_{t} \mid x_{1}, \ldots, x_{T}\right)=0 \quad(t=1, \ldots . T),
\end{aligned}
$$

where

$$
c_{1}=\delta_{2} x_{0}+\delta_{3} y_{0}+\delta_{4} y_{-1}, c_{2}=y_{0},
$$

and there are nonlinear restrictions on the parameters. . The $\underset{\sim}{\mathbb{m a t r}} \mathbf{i x}$ has the following form: 


$$
\underset{\sim}{\pi}=\underset{\sim}{B}+\underset{\sim}{Y_{1}} \lambda_{1}^{\prime}+\underset{\sim}{\gamma_{2}} \lambda_{2}^{\prime}
$$

where $B$ is lower triangular, $Y_{j}^{\prime}=\left(Y_{1 j}, \ldots,{ }_{T j}^{Y}\right)$, and $E^{*}\left(c_{j} \mid x\right)={\underset{\sim j}{j}}_{\mathbf{z}}(j=1,2)$

This specification suggests a natural extension of the conditional

strict exogeneity idea, with the conditioning set indexed by the number of latent variables. We shall say that " $\mathbf{x}$ is strictly exogenous conditional on $c_{1}, c_{2} "$ if

$$
E^{*}\left(y_{t} \mid \ldots, x_{t-1}, x_{t}, x_{t+1}, . . ., c_{1}, c_{2}\right)=E^{*}\left(y_{t} \mid x_{t}, x_{t-1}, \ldots, c_{1}, c_{2}\right) .
$$

We can also introduce a Granger version of this condition and generalize the analysis in section 2.5 .

Serial Comelation or Partial Adjustment?

Griliches' (1967) considered the problem of distinguishing between

the following two models: a partial adjustment model, 16

$$
y_{t}=\beta x_{t}+\gamma y_{t-1}+\nabla_{t}
$$

and a model with no structural lagged dependent variable but with a residual following a first order Markov process:

$$
\begin{aligned}
& y_{t}=\beta x_{t}+u_{t}, \\
& u_{t}=\rho u_{t-1}+e_{t}, e_{t} \text { I.1.d.; }
\end{aligned}
$$

in both cases $\mathbf{x}$ is strictly exogenous:

$$
E^{*}\left(v_{t} \mid x_{1}, \ldots . x_{T}\right)=E^{*}\left(u_{t} \mid x_{1}, \ldots . x_{T}\right)=0 \quad(t=1, \ldots . T)
$$


In the serial correlation case, we have

$$
y_{t}=\beta x_{t}-\rho \beta x_{t-1}+\rho y_{t-1}^{\prime}+e_{t}
$$

as Griliches observed, the least squares regression will have a distinctive pattern -- the coefficient on lagged $\mathbf{x}$ equals (as $N \rightarrow \infty$ ) minus the product of the coefficients on current $\mathbf{x}$ and lagged $y$.

I want to point out that this prediction does not rest on the serial correlation structure of $\mathbf{u}$. It is a direct implication of the assumption' that $u$ is uncorrelated with $x_{1}, \ldots . . x_{T}:$

$$
\begin{aligned}
E *\left(y_{t} \mid x_{t}, x_{t-1}, y_{t-1}\right) & =\beta x_{t}+E *\left(u_{t} \mid x_{t}, x_{t-1}, y_{t-1}\right) \\
& =\beta x_{t}+E *\left(u_{t} \mid u_{t-1}\right) \\
& =\beta x_{t}+\varphi t u_{t-1} \\
& =\beta x_{t}-\varphi \theta_{t}^{\beta} x_{t-1}+\varphi t_{t-1}^{y} .
\end{aligned}
$$

Here $\boldsymbol{v}_{\mathbf{t}} \mathbf{u}_{\mathbf{t}-\mathbf{1}}$ is simply notation for the linear predictor. In general $\mathbf{u}_{\mathbf{t}}$ is not a first order process $\left(\mathbf{E}^{*}\left(\mathbf{u}_{t} \mid \mathbf{u}_{t-1}, \mathbf{u}_{t-2}\right) \notin \mathbf{E}^{*}\left(\mathbf{u}_{t} \mid \mathbf{u}_{t-1}\right)\right)$, but this does not affect our argument.

Within the $\underset{\sim}{\mathbb{\tau}}$ matrix framework, the distinction between the two models is that (2.14) implies a diagonal $\underset{\sim}{m}$ matrix, with no distributed lag, whereas the partial adjustment specification in (2.13) implies that $\underline{\boldsymbol{I}} \mathbf{\mathbf { B }}+\mathbf{Y}_{\sim} \boldsymbol{\lambda}_{\sim}^{\prime}$, with a distributed lag in the lower triangular $\underset{\sim}{\mathbf{B}}$ matrix and a rank one set of lags and leads in $\underline{\gamma}_{\sim}^{\prime}$.

We can generalize the serial correlation model to allow for an individual specific effect that may be correlated with $\mathrm{x}$ : 


$$
\begin{aligned}
& y_{t}=\beta x_{t}+c+u_{t}, \\
& E *\left(u_{t} \mid x_{I}, \ldots . \cdot x_{T}\right)=0 .
\end{aligned}
$$

Now both the serial correlation and the partial adjustment models have a rank one sat of lags and leads in $\underset{\sim}{\mathbb{2}}$, but we can distinguish between them because on ily the partial adjustment model has a distributed lag in the $\mathbf{B}$ matrix. So the absence of structural lagged dependent variables is signalled by the following special case of conditional strict exogeneity:

$$
E^{*}\left(y_{t} \mid x_{1}, \ldots, x_{T}, c\right)=E^{*}\left(y_{t} \mid x_{t}, c\right)
$$

In this case the relationship of $\mathbf{x}$ to $y$ is "static" conditional on $\mathbf{c}$. We shall purs ie this distinction in nonlinear models in section 3.3.

\subsection{Resi al Covariances: Heteroskedasticity and Semial Correlation}

\section{7.a. Beteroskedastioity}

$$
\text { If } \mathbf{E}\left(\boldsymbol{c}_{\mathbf{1}} \mid \mathbf{x}_{\mathbf{1}}\right)+\mathbf{E}^{*}\left(\mathbf{c}_{\mathbf{1}} \mid \mathbf{x}_{\mathbf{1}}\right) \text {, then there will be heteroskedasticity, }
$$

since the residual will contain $\mathbf{E}\left(\mathbf{c}_{\mathbf{1}} \mid \mathbf{x}_{\mathbf{1}}\right) \cdot \mathbf{E}^{*}\left(\mathbf{c}_{\mathbf{1}} \mid \mathbf{x}_{\mathbf{1}}\right)$. Another source of heteroskedasticity is random coefficients:

$$
\begin{aligned}
& y_{1 t}=b_{1} x_{1 t}+c_{1}+u_{i t}, \\
& b_{1}=\beta+w_{i}, E\left(w_{1}\right)=0, \\
& y_{i t}=\beta x_{1 t}+c_{i}+\left(w_{1} x_{1 t}+u_{1 t}\right) .
\end{aligned}
$$

If $\boldsymbol{w}$ is independent of $x$, then $\underset{\sim}{\pi}=\boldsymbol{B} \underset{\sim}{\mathbb{I}}+\underline{\boldsymbol{\ell}} \underline{\boldsymbol{\lambda}}^{\prime}$, and our previous discussion, is relevant for the estimation of $\boldsymbol{B}$. We shall handle the heteroskedasticity 


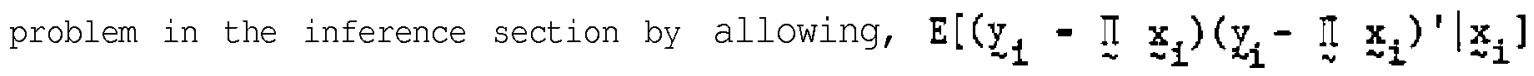
to be an arbitrary function of $\mathbf{x}_{\mathbf{i}} \cdot 17$

\section{7.b. Serial Correlation}

It may be of interest to impose restrictions on the residual covariances, such as a variance-components structure together with an autoregressive-moving average scheme. ${ }^{18}$ consider the homoskedastic case in which

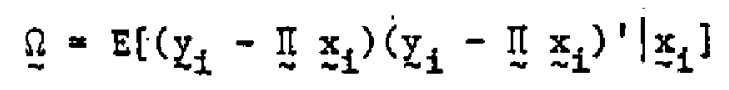

does not depend upon $\mathbf{x}_{\mathbf{1}}$. Then the restrictions can be expressed as $\Omega_{j k}=g_{j k}(\stackrel{\theta}{\sim})$, where the $g^{\prime} s$ are known functions and $\underset{\sim}{\theta}$ is an unrestricted parameter vector. We shall discuss a minimum distance procedure for imposing such restrictions in the inference section.

3. SPECIFICATION AND IDENTIFICATION: NONLINEAR MODELS

\subsection{A Random Effects Probit Modez}

Our treatment of individual effects carries over with some important qualifications to nonlinear models. We shall illustrate with a labor force participation example. If the upper bound on leisure is binding in (2.6), then

$$
\rho^{(t-1)} \tilde{U}_{t}(\bar{L})>m r^{-(t-1)} W_{t},
$$

where $\mathbf{m}$ is the Lagrange multiplier corresponding to the lifetime budget constraint (the marginal utility of initial wealth). Let $y_{i t}=1$ if individual $i$ works in period $t, y_{i t}=0$ otherwise. Let 


$$
\begin{aligned}
& \text { lit } W_{t}=\varphi_{1} x_{i t}+e_{1 i t}, \\
& \text { ln } A_{i t}=\varphi_{2} x_{i t}+e_{2 i t},
\end{aligned}
$$

where $x_{\text {it }}$ contains measured variables that predict wages and tastes for leisure. We shall simplify the notation by supposing that $\boldsymbol{x}_{\text {it }}$ consists of a single variable. Then $\mathrm{y}_{\text {it }}=1$ if

$$
\begin{aligned}
\left(\varphi_{1}-\varphi_{2}\right) \mathbf{x}_{i t} & -(t-1) \ln (\gamma \rho)+\text { en } \mathbf{m}_{1} \\
& +(1-6) \text { en } \bar{L}+e_{11 t}-e_{21 t} \geq 0,
\end{aligned}
$$

which we shall write as

$$
\beta x_{1 t}+\varphi(t-1)+c_{i}+u_{1 t} \geq 0
$$

Now we need a distributional assumption for the $\mathbf{u}$ 's. We shall assume that $\left(u_{\prime \prime}, . . . u_{T}\right)$ is independent of $c$ and the $x^{\prime} s$, with a multivartate normal distribution $(\mathbb{N}(0, \Sigma))$. So we have a problt model (suppressing the 1 subscripts and period-specific intercepts):

$$
P\left(y_{t}=1 \mid x_{1}, \ldots . . x_{T}, c\right)=F\left[\sigma_{t t}^{-\frac{1}{2}}\left(\beta x_{t}+c\right)\right]
$$

where $\boldsymbol{F}()$ is the standard normal distribution function and $\sigma_{\mathbf{t t}}$ is the th $\underline{\text { th }}$ diagonal element of $\underline{\Sigma}$.

Next we shall specify a distribution for c conditional on $\mathbf{x} \cdot\left(x_{1}, .+. \quad \cdot \mathbf{x}_{T}\right)$ :

$$
c=\psi+\lambda_{1} x_{1}+\ldots \cdot+\lambda_{T} x_{T}+v
$$

where $v$ is independent of the $x^{\prime} s$ and has a normal distribution $\left(\mathbf{N}\left(0, \sigma_{v}^{2}\right)\right.$ ). 
There is a very important difference in this step compared with the linear case. In the linear case it was not restrictive to decompose $c$ into $\mathbf{1 t s}$ linear projection on $\mathrm{x}$ and an orthogonal residual. Now, however, we are assuming that the regression function $\mathbf{E}(\mathbf{c} \mid \mathbf{x})$ is actually linear, that $\mathbf{v}$ is independent of $\mathbf{x}$, and that $\mathbf{v}$ has a normal distribution. These are restrictive assumptions and there may be a payoff to relaxing them.

Given these assumptions, the distribution for $y_{t}$ conditional on $\mathbf{x}_{\mathbf{1}}, \ldots . \mathbf{x}_{\mathbf{T}}$ but marginal on $\mathbf{c}$ also has a problt form:

$$
\begin{aligned}
& P\left(y_{t}=1 \mid x_{1}, \ldots . x_{T}\right)=F\left[\alpha_{t}\left(\beta x_{t}+\lambda_{1} x_{1}+\ldots .+\lambda_{T} x_{T}\right)\right], \\
& \alpha_{t}=\left(\sigma_{t t}+\sigma_{v}^{2}\right)^{-\frac{1}{2}} .
\end{aligned}
$$

Combining these $T$ specifications gives the following watrix of coefficients: 19

$$
\pi=\operatorname{diag}\left\{\alpha_{1}, \ldots . . \alpha_{T}\right\}\left[\beta I_{\tau T}+\ell \lambda^{\dagger}\right]
$$

This differs from the linear case only in the diagonal matrix of normalization factors $\boldsymbol{\alpha}_{\boldsymbol{t}}$. There are now nonlinear restrictions on $\mathbb{\boldsymbol { \pi }}$, but the identification analysis is still straightforward. We have

$$
\begin{aligned}
& \alpha_{t} \beta=\frac{\alpha_{t}}{\alpha_{1}} a_{11}=\pi_{t 1}=\pi_{t t}=\frac{\alpha_{t}}{\alpha_{1}} \pi_{1 t}, \\
& \frac{\alpha_{t}}{\alpha_{1}}=\left(\pi_{t t}+\pi_{t 1}\right) /\left(\pi_{11}+\pi_{1 t}\right) \quad(t-2, \ldots . . T),
\end{aligned}
$$

if $\beta+\lambda_{1}+A_{1} \neq 0$. Then, as in the linear case, we can solve for $\alpha_{1} \beta$ and $\alpha_{1} \lambda$. Only ratios of coefficients are identified, and so we can use a scale normalization such as $\alpha_{1} \equiv 1$. 
ds for inference, a computationally simple approach is to estimate $\mathrm{I}$ cross-sectional probit specifications by maximum likelihood, where $x_{1}, \ldots, x_{T}$ are included in each of the $T$ specifications. This gives $\hat{\pi}_{n}(t=1, \ldots . . T)$ and we can use a Taylor expansion to derive the covariance matrix of the asymptotic normal distribution for $\left(\hat{\pi}_{\sim 1}, . . \cdot \hat{\pi}_{\sim T}\right)$. Then restrictions can be imposed on II using a minimum distance estimator, just as in the linear case.

We shall conclude our discussion of this model by considering the interpretation of the coefficients. We began with the probit specification that

$$
P\left(y_{t}=1 \mid x_{1}, \ldots . X X T, c\right)=F\left[\sigma_{t t}^{-\frac{1}{2}}\left(\beta x_{t}+c\right)\right]
$$

So one might argue that the correct measure of the effect of $\mathbf{x}_{\mathbf{t}}$ is based on $\sigma_{t t}^{-\frac{1}{2}} \beta$, whereas we have obtained $\left(\sigma_{t t}+\sigma_{v}^{2}\right)^{-\frac{1}{2} \beta}$, which is then an underestimate. But there is something curious about this argument, since the "omitted variable" $\mathbf{v}$ is independent of $\mathbf{x}_{\mathbf{1}}, . . . \mathbf{x}_{\mathbf{T}}$. Suppose that we decompose $\mathbf{u}_{\mathbf{t}}$ in $\left(3.1\right.$ ) into $\mathbf{u}_{\mathbf{I t}}+\mathbf{u}_{\mathbf{2}}$ and that measurements on $\mathbf{u}_{\mathbf{I t}}$ become available. Then this argument implies that the correct measure of the effect of $\mathbf{x}_{\mathbf{t}}$ is based on $\left[\mathbf{V}\left(\mathbf{u}_{2 t}\right)\right]^{-\frac{1}{2}} \boldsymbol{B}$. As the data collection becomes increasingly successful. there is less and lass variance left in the residual $\mathbf{u}_{2 t}$, and $\left[\mathbf{V}\left(\mathbf{u}_{2 t}\right)\right]^{-\frac{T}{2}}$ becomes arbitrarily large.

The resolution of this puzzle is that the effect of $\mathbf{x}_{\mathbf{t}}$ depends upon the value of $\mathbf{c}$, and the effect evaluated at the average value for $\mathbf{c}$ is not equal to the average of the effects, averaging over the distribution for $c$. Consider the effect on the probability that $\mathbf{y}_{\mathbf{t}} \mathbf{*}$ of increasing $\mathbf{x}_{\mathbf{t}}$ from $\mathbf{x}^{\prime}$ to $\mathbf{x}^{\prime \prime}$; using the average value for $\mathbf{c}$ gives 


\section{6}

$$
F\left[\sigma_{t t}^{-\frac{x}{2}}\left(\beta x^{\prime \prime}+E(c)\right)\right]=F\left[\sigma_{t t}^{-\frac{1}{z_{2}}}\left(\beta x^{\prime}+E(c)\right)\right]
$$

The problem with this measure is that it may be relevant for only a small fraction of the population. I think that a more appropriate measure is the mean effect for a randomly dram individual:

$$
\int\left[P\left(y_{t}=1 \mid x_{t}=x^{\prime \prime}, c\right)-P\left(y_{t}=1 \mid x_{t}=x^{\prime}, c\right)\right] \mu(d c)
$$

where $\mu(d c)$ gives the population probability measure for $c$.

We shall see how to recover this measure within our framework. Let $\mathbf{z}=\lambda_{1} x_{1}+\ldots+\lambda_{T} \mathbf{x}_{T} ;$ let $\mu(\mathbf{d z})$ and $\mu(d \mathbf{v})$ give the population probability measures for the independent random variables $\mathbf{z}$ and $\mathbf{v}$. Then

$$
\begin{aligned}
& P\left(y_{t}-1 \mid x_{t}, c\right)=P\left(y_{t}=1 \mid x_{1}, \ldots, x_{T}, c\right) \\
& =P\left(y_{t}=1 \mid x_{t}, z, v\right) ; \\
& \int P\left(y_{t}=1 \mid x_{t}, z, v\right) \mu(d z) \mu(d v) \\
& =\int P\left(y_{t}=1 \mid x_{t}, z, v\right) \mu\left(d v \mid x_{t}, z\right) \mu(d z) \\
& =\int P\left(y_{t}=1 \mid x_{t}, z\right) \mu(d z)
\end{aligned}
$$

where $\mu\left(\mathbf{d} \mathbf{v} \mid \mathbf{x}_{\mathbf{t}}, \mathbf{z}\right)$ is the conditional probability measure, which equals the unconditional measure since $\mathbf{v}$ is independent of $\mathbf{x}_{\mathbf{t}}$ and $\mathbf{z}$. ( $\mathbf{t}$ is important to note that the last integral does $\underline{\text { not }}$, in general, equal $\mathbf{P}\left(\mathbf{y}_{\mathbf{t}}=\mathbf{1} \mid \mathbf{x}_{\mathbf{t}}\right)$. For if $\mathbf{x}_{\mathbf{t}}$ end $\mathbf{z}$ are correlated, as they are in our case, then

$$
\begin{aligned}
& P\left(y_{t}=1 \mid x_{t}\right)=\int P\left(y_{t}=1 \mid x_{t}, z\right) \mu\left(d z \mid x_{t}\right) \\
& \left.\quad f \int P\left(y_{t}=1 \mid x_{t}, z\right) \mu(d z) .\right)
\end{aligned}
$$


We have shown that

$$
\begin{aligned}
& \int\left[P\left(y_{t}=1 \mid x_{t}=x^{\prime \prime}, c\right)-P\left(y_{t}=1 \mid x_{t}=x^{\prime}, c\right)\right] \mu(d c) \\
& \quad=\int\left[P\left(y_{t}=1 \mid x_{t}=x^{\prime \prime}, z\right)-P\left(y_{t}=1 \mid x_{t}=x^{\dagger}, z\right)\right] \mu(d z) .
\end{aligned}
$$

The integration with respect to the marginal distribution for $\mathbf{z}$ can be done using the empirical distribution furiction, which gives the following consistent (as $N \rightarrow \infty$ ) estimator of (3.15)

$$
\begin{aligned}
\frac{1}{N} \sum_{i=1}^{N} & \left\{F\left[\alpha_{t}\left(\beta x^{\prime \prime}+\lambda_{1} x_{11}+\ldots+\lambda_{T} x_{1 T}\right)\right]\right. \\
& \left.-F\left[\alpha_{t}\left(\beta x^{\prime}+\lambda_{1} x_{11}+\ldots .+\lambda_{T} x_{i T}\right)\right]\right\} .
\end{aligned}
$$

\subsection{A Fixed Effects Logit Model: Conditional Likelihood}

'A weakness in the probit model was the specification of a distribution for $c$ conditional on $x$. A convenient form was chosen, but it was only an approximation, perhaps a poor one. We shall discuss a technique that does not require us to specify a particular distribution for $\mathbf{c}$ conditional on x; it will, however, have its own weaknesses. Consider the following specification:

$$
P\left(y_{t}=1 \mid x_{1}, \ldots . . x_{T}, c\right)=G\left(\beta x_{t}+c\right), G(z)=e^{z} /\left(I+e^{z}\right),
$$

where $\mathbf{y}_{\mathbf{I}}, \ldots . \mathrm{y}_{\mathbf{T}}$ are independent conditional on $\mathbf{x}_{\mathbf{1}}, \ldots . . \mathbf{x}_{\mathbf{T}}, \mathbf{c}$.

Suppose that $\mathbf{T}=\mathbf{2}$ and compute the probability that $\mathbf{y}_{\mathbf{2}}=1$ conditional, on $y_{1}+y_{2}=1:$

$$
P\left(y_{2}=1\left\{x_{1}, x_{2}, C, y_{1}+y_{2} \cdot I 1\right)=G\left[\beta\left(x_{2}-x_{1}\right)\right]\right.
$$


which does not depend upon c. Given a random sample of individuals, the conditional log-likelihood function is

$$
L=\sum_{i=B}\left\{w_{i} \ln G\left[B\left(x_{i 2}-x_{i 1}\right)\right]+\left(1-w_{i}\right) \ln G\left[-B\left(x_{i 2}-x_{i 1}\right)\right]\right\},
$$

where

$$
\begin{aligned}
& w_{i}=\left\{\begin{array}{l}
1 \text { if }\left(y_{i 1}, y_{i 2}\right)=(0,1) \\
0 \text { if }\left(y_{11}, y_{i 2}\right)=(1,0),
\end{array}\right. \\
& B=\left\{i \mid y_{i 1}+y_{i 2}=1\right\} .
\end{aligned}
$$

This conditional likelihood function does not depend upon the incidental parameters. It is in the form of a binary logit likelihood function in which the two outcomes are $(0.1)$ and $(1,0)$ with es rlanatory variables $\mathbf{x}_{2}-\mathbf{x}_{1}$. This is the analog of differencing in the wo period linear model. The conditional maximm likelihood (ML) estrmat? of $\beta$ can be obtained simply from a ML binary logit program. This conditional likelihood approach was used by Rasch $(1960,1961)$ in his model for inteligence tests. 20

The conditional ML estimator of $\beta$ is consistent provided that the conditional likelihood function satisfies regularity conditions, which impose mild restrictions on the $\boldsymbol{c}_{\boldsymbol{1}}$. These restrictions, which are satisfied if the $\mathrm{ci}$ are a random sample from some distribution, are discussed in Andersen (1970). Furthermore, the inverse of the information' matrix based on the conditional likelihood function provides a covarfance matrix for the asymptotic $(\mathbb{N} \rightarrow \infty)$ normal distribution of the conditional ML estimator of $\beta$.

These results should be contrasted with the inconsistency of che standard fixed effects $\mathbf{i}$ estinator, in which the likelihood function is 
based on the distribution of $\mathrm{y}_{1}, \ldots . \mathrm{y}_{\mathrm{T}}$ conditional on $\mathrm{x}_{\mathrm{L}}, \ldots . . \mathrm{xT}, \mathrm{c}$. For example, suppose that $T=2, x_{i 1}=0, x_{i 2}=1(i=1, \ldots . . N)$. The following limits exist with probability one if the $c_{i}$ are a random sample from some distribution:

$$
\begin{aligned}
& \lim _{\mathbb{N} \rightarrow \infty} \frac{1}{N} \sum_{i=1}^{N} E\left[y_{i 1}\left(1-y_{i 2}\right) \mid c_{i}\right]=\varphi_{1}, \\
& \lim _{N \rightarrow \infty} \frac{1}{N} \sum_{i=1}^{N} E\left[\left(1-y_{i 1}\right) y_{12} \mid c_{1}\right]=\varphi_{2},
\end{aligned}
$$

where

$$
\begin{aligned}
& E\left[y_{11}\left(1-y_{i 2}\right) \mid c_{1}\right]=G\left(c_{1}\right) G\left(-\beta-c_{i}\right), \\
& E\left[\left(1-y_{11}\right) y_{12} \mid c_{1}\right]=G\left(-c_{1}\right) G\left(\beta+c_{i}\right) .
\end{aligned}
$$

Andersen (1973, P. 66) shows that the ML estimator of $\beta$ converges with probability one to $2 B$ as $N \rightarrow \infty$. A simple extension of his argument shows that if $G$ is replaced by any distribution function ( $\tilde{G}$ ) corresponding to a symmetric, continuous, nonzero probability density, then the ML estimator of 3 converges with probability one to

$$
2 \tilde{G}^{-1}\left(\frac{\varphi_{2}}{\varphi_{1}^{+\varphi_{2}}}\right) \text {. }
$$

The logit case is special in that $\varphi_{2} / \varphi_{1}=\mathbf{e}^{\beta}$ for any distribution for $c$. In general the limit depends on this distribution; but if all of the $\boldsymbol{c}_{\mathbf{1}} \mathbf{x} 0$, then once again we obtain convergence to 26 as $N \rightarrow \infty$.

For general $\mathrm{T}$, conditioning on $\Sigma_{t^{y t}}(1=1, \ldots . N)$ gives the following conditional log-likelihood function: 


$$
\begin{aligned}
& L=\underset{i=1}{i} \ln \left[\exp \left(\beta \sum_{t=1}^{T} x_{i t} y_{i t}\right) / \sum_{d \in B} \exp \left(B \sum_{t=1}^{T} x_{i t} d_{t}\right) I\right. \\
& B_{i}=\left\{d=\left(d_{1}, \ldots . . d_{T}\right) \mid d_{t}=0 \text { or } 1 \text { and } \sum_{t=1}^{T} d_{t}=\sum_{t=1}^{T} y_{i t}\right\} .
\end{aligned}
$$

I is in the conditional logit form considered by McFadden (1974), with the alternative set $\left(\mathbf{B}_{\mathbf{f}}\right)$ varying across the observations. Hence it can be maximized by standard programs. There are $\mathbf{T}+1$ distinct alternative sets corresponding to $\Sigma_{t} y_{i t}=0,1, \ldots, T$. Groups for which $\Sigma_{t} y_{i t}=0$ or $T$ contribute zero to $\mathrm{I}$, however, and so only $\mathrm{I}-\mathrm{l}$ alternative sets are relevant. The alternative set for the group with $\Sigma_{\mathbf{t}} \mathbf{y}_{\mathbf{1 t}}=\mathrm{s}$ has $\left(\begin{array}{l}\mathrm{T} \\ \mathbf{s}\end{array}\right)$ elements, corresponding to the distinct sequences of $T$ tI als with $\mathbf{s}$ successes. For example, with $\mathbf{r}=\mathbf{3}$ and $\mathbf{s} \mathbf{= 1}$ there are three alter atives with the following conditional p'obabilities :

$$
\begin{aligned}
& P\left(1,0,0 \mid \underline{x}_{1}, c_{1}, \sum_{t} Y_{1 t}=1\right)=\exp \left[B\left(x_{11}-x_{13}\right)\right] / D \\
& P\left(0,1,0 \mid \underline{x}_{1}, c_{i}, \sum_{t}^{\sum} Y_{i t}=1\right)=\exp \left[B\left(x_{12}-x_{13}\right)\right] / D \\
& P\left(0,0,1{\mid x_{1}}_{1} c_{1}, \sum_{t} Y_{1 t}=I\right)=1 / D \\
& D=\exp \left[B\left(x_{11}-x_{i 3}\right) I+\exp \left[B\left(x_{12}-x_{13}\right)\right]+1 .\right.
\end{aligned}
$$


A weakness in this approach is that it relies on the assumption that the $y t$ are independent conditional on $\mathbf{x}, \mathbf{c}$, with an identical form for the conditional probability each period: $P\left(y_{t}=1 \mid \underline{x}, c\right)=G\left(3 x_{t}+c\right)$. In the probit framework, these assumptions translate into $\Sigma=\sigma^{2}$ I, so that $\mathbf{v}+\mathbf{u}_{\mathbf{t}}$ generates an equicorrelated matrix: $\sigma_{\mathbf{v}}^{2} \underline{\ell} \ell^{\prime}+\sigma^{2} \mathrm{I}$. We have seen that it is straightforward to allow $\Sigma$ to be unrestricted in the probit framework; that is not true here.

An additional weakness is that we are limited in the sorts of probability statements that can be made. We obtain a clean estimate of the effect of $\mathbf{x}_{t}$ on the $\log$ odds:

$$
\ln \left[\frac{P\left(y_{t}=1 \mid x_{t}=x^{\prime \prime}, c\right)}{P\left(y_{t}=0 \mid x_{t}=x^{\prime \prime}, c\right)} / \frac{P\left(y_{t}=1 \mid x_{t}=x^{\prime}, c\right)}{P\left(y_{t}=0 \mid x_{t}=x^{\prime}, c\right)}\right]=B\left(x^{\prime \prime}-x^{\prime}\right)
$$

the special feature of the logistic functional form is that this function of the probabilities does not depend upon $c$; so the problem of integrating over the marginal distribution of $\mathbf{c}$ (instead of the conditional distribution of $\boldsymbol{c}$ given $\boldsymbol{x}$ ) does not arise. But this is not the only function of the probabilities that one might want to know. In the probit section we considered

$$
P\left(y_{t}=1 \mid x_{t}=x^{\prime \prime}, c\right)-P\left(y_{t}=1 \mid x_{t}=x^{\prime}, c\right)
$$

which depends upon $\mathbf{c}$ for probit or logit, and we averaged over the marginal distribution for $\mathrm{c}$ :

$$
\int\left[P\left(y_{t}=1 \mid x_{t}=x^{\prime \prime}, c\right)-P\left(y_{t}=1 \mid x_{t}=x^{\prime}, c\right)\right] \mu(d c)
$$


This requires us to specify a marginal distribution for $c$, which is what the conditioning argument trys to avoid. We cannot estimate (3.7) if all we have is the conditional yL estimate of $\beta$.

Our specification in (3.5) asserts that $y_{t}$ is independent of $\mathbf{x}_{1}, \ldots . \mathbf{x}_{t-1}, \mathbf{x}_{t+1}, \ldots . x_{T}$ conditional on $x_{t}, c$. This can be relaxed somewhat, but the conditional likelihood argument certainly requires more than

$$
P\left(y_{t}=1 \mid x_{t}, c\right)=G\left(B x_{t}+c\right)
$$

to see this, try to. derive (3.6) with $x 2=y_{1}$, we can, however, implement the following specification (with $\underline{x}^{\prime}=\left(x_{1}, . . . . x_{T}\right)$ ):

$$
P\left(y_{t}=I \mid x, c\right)=G\left(\beta_{t o}+\beta_{t 1} x_{1}+\cdots+\beta_{t t} x_{t}+c\right)
$$

where $\mathrm{y}_{\mathbf{1}}, \ldots \cdot \mathrm{y}_{\mathbf{T}}$ are independent conditional on $\underset{\sim}{\mathbf{x}}, \mathrm{c}$. This corresponds to our specification of " $\mathbf{x}$ is strictly exogenous conditional on $\mathbf{c}$ " in Section 2.5, except that $\boldsymbol{Y}_{\mathbf{t}}=1$ in the term $\boldsymbol{\gamma}_{\mathbf{t}} \mathbf{c}^{--}$it is not strafghtforward to allow a time-varying coefficient on $\mathbf{c}$ in the conditional likelihood approach. The extension of (3.6) $1 \mathrm{~s}$

$$
\begin{gathered}
P\left(y_{t}=1 \mid x, c, y_{1}+y_{t}=I\right)=G\left(\tilde{\beta}_{t o}+\tilde{\beta}_{t 1} x_{1}+\beta_{t 2} x_{2}+\ldots+\beta_{t t} x_{t}\right) \\
(t=2, \ldots . T),
\end{gathered}
$$

where $\beta_{t j}=\beta_{t j}-\beta_{1 j}(j=0,1)$. So if $\mathbf{x}$ has sufficient variation, we can obtain consistent estimates of $\tilde{\beta}_{\mathbf{t o}}, \tilde{\beta}_{\mathbf{t l}}$, and $\boldsymbol{\beta}_{\mathbf{t s}}(\mathbf{s}=\mathbf{2}, \ldots \mathbf{t})$. 
only these parameters are identified, since we can transform the model replacing $a$ by $L=\beta_{i 0}+B_{i l} x_{1}+c$ without violating any restrictions.

The restrictions in (3.5) or in (3.8) can be tested against the following alternative:

(3.10) NY, $=1 \mid \mathbf{x}, c)=G\left(\pi_{t o}+\pi_{t l} \mathbf{x}_{1}+\ldots .+\pi_{t T_{T}} x_{T}+c\right)$.

We can identify only $\pi_{\mathbf{t j}}=\pi_{\mathbf{l j}}$ and so we can normalize $\pi_{1 \boldsymbol{j}}=0 \quad(\boldsymbol{j}=\mathbf{0}, \ldots, \mathbf{T}$; $\mathbf{t}=\mathbf{2}, \ldots . \mathbf{T})$. The maximized values of the conditional log likelihoods can be used to form $x^{2}$ statistics. 21 There are $(\mathbf{T}-\mathbf{2})(\mathbf{T}-\mathbf{1}) / 2$ restrictions in passing from (3.10) to (3.8), and (3.5) imposes an additional $(\mathrm{T}-1)(\mathrm{T}+4) / 2-1$ restrictions.

\subsection{Serial Compelation And Lugged Dependent Variables} Consider the following two models:

(3.11a) $y_{t}=\left\{\begin{array}{l}1 \text { if } y_{t}^{*}=y_{t-1}+e_{t} \geq 0 \\ 0 \text { otherwise, }\end{array}\right.$

(3.IIb) $y_{t}=\left\{\begin{array}{l}1 \text { if } y_{t}^{*}=u_{t} \geq 0 \\ 0 \text { otherwise; } u_{t}=\rho u_{t-I}+e_{t} ;\end{array}\right.$ 
in both cases et is i.i.d. $\mathbf{H}\left(0, \sigma^{2}\right)$. Heckman (1978) observed that we can distinguish between these two models. 22 In the first model,

$$
P\left(y_{t}=1 \mid y_{t-1}, y_{t-2}, \ldots\right)=P\left(y_{t}=1 \mid y_{t-1}\right)=F\left(\gamma y_{t-1} / \sigma\right),
$$

where $\mathbf{F}()$ is the standard normal distribution function. In the second model, however, $\mathbf{P}\left(\mathbf{y}_{\mathbf{t}}=1 \mid y_{t-1}, y_{t-2}, . ..\right)$ depends upon the entire history of the process. If we observed $\mathbf{u}_{\mathbf{t}-\mathbf{I}}$, then previous outcomes would be irrelevant. In fact, we observe only whether $u_{t-1} \geq 0$; hence conditioning in addition on whether $\mathbf{u}_{\mathbf{t}-\mathbf{2}} \geq 0$ affects the distribution of $\mathbf{u}_{\mathbf{t}-\mathbf{1}}$ and $\mathbf{y}_{\mathbf{t}} \cdot$ So the lagged $y$ implies a Markov chain whereas the Markov assumption for the probit residual does not imply a Markov chain for the binary sequence that it generates.

There is an analogy with the following linear models:

$(3.12 a)$

$$
\therefore y_{t}=\gamma y_{t-1}+e_{t}
$$

$$
y_{t}=u_{t}, u_{t}=e_{t}+P e_{t-1}
$$

where $\mathbf{e}_{\mathbf{t}}$ is i.i.d. $\mathbf{s}\left(0, \sigma^{2}\right)$. We know that if $\mathbf{u}_{\mathbf{t}}=\boldsymbol{\rho}_{\mathbf{t}-\mathbf{1}}+\mathbf{e}_{\mathbf{t}}$, then no distinction would be possible, without introducing more structure, since both models imply a linear Markov process. With the moving average residual, however, the serial correlation model implies that the entire past history is relevant for predicting $y$. So the distinction between the two models rests on the order of the dependence on previous realizations of $y_{E}$. We can still distinguish between the two models even when $\left(\mathbf{u}_{\mathbf{1}}, \ldots . . \mathbf{u}_{\mathbf{T}}\right)$ has a general multivarlate normal distribution $(\mathbb{N}(\underset{\sim}{\mu}, \Sigma))$. Given nor- 
malizations such as $\mathrm{V}\left(\mathrm{u}_{t}\right)=1(\mathrm{e}-1, \ldots . \mathrm{T})$, the serial correlation model has $\mathrm{T}(\mathrm{T}+1) / 2$ free parameters. Hence if $\mathrm{T} \geq 3$, there are restrictions on the $2^{I}$ - 1 parameters of the multinomial distribution for $\left(\mathrm{y}_{n}, \ldots . . \mathrm{y}_{\mathrm{T}}\right)$. In particular, the most general multivariate probit model cannot generate a Markov chain. So we can add a lagged dependent variable and identify $Y$.

This result relies heavily on the restrictive nature of the multivariate probit functional form. A more robust distinction between the two models is possible when there is variation over time in $\mathbf{x}_{\mathbf{t}} \cdot$ We shall pursue this after first presenting a generalization of strict exogeneity and noncausality for nonlinear models.

Let $t=1$ be the first period if the individual's (economic) life. An extension of Granger's definition uf " $y$ does not cause $\mathbf{x}^{\prime \prime}$ is that $\mathbf{x}_{\mathbf{t + 1}}$ is independent of $\mathbf{y}_{1}, \ldots . \mathrm{y}_{\mathbf{t}}$ condit onal on $\mathbf{x}_{\mathbf{1}}, \ldots \cdot \mathbf{x}_{\mathbf{t}} \cdot$ An extension of Sims' strict exogeneity condition is thst $\mathbf{y}_{t}$ is independent of $\mathbf{x}_{t+1}, \mathbf{x}_{t+2}, .$. . conditional on $\mathbf{x}_{\mathbf{1}}, \ldots, \mathbf{x}_{\mathbf{t}}$. In contrast to the linear predictor case, these two definitions are no longer equivalent. 23 For consider the following counterexample: let $\mathrm{y}_{1}, \mathrm{y}_{2}$ be independent Bernoulli random variables with $P\left(y_{t}=1\right)=P\left(y_{t}=-1\right)=1 / 2(t=1,2)$. Let $x 3=y_{1} y_{2}$. Then $y_{1}$ is independent of $x 3$ and $\mathbf{y}_{2}$ is independent of $\mathbf{x}_{3}$. Let all of the other random variables be degenerate (equal to zero, say). Then $\mathbf{x}$ is strictly exogenous but $\mathrm{x} 3$ is clearly not independent of $\mathrm{y}_{1}, \mathrm{y}_{2}$ conditional on $\mathrm{xl}, \mathbf{x}_{2}$. The counterexample works for the following reason: if a random variable is uncorrelated with each of two other random variables, then it is uncorrelated with every linear combination of them; but If it is independent of each of the other random variables, it need not be independent of every function of them. 
Consider the following modification of Sims' condition: $y_{t}$ is independent of $x_{t+1}, x_{t+2}, \ldots$ conditional on $x_{1}, \ldots, x_{t}, y_{1}, \ldots, y_{t-1}$ $(t=1,2, \ldots)$. Chamberlain (1982) shows that, subject to a regularity condition, this is equivalent to our extended definition of Granger noncausality. The regularity condition is trivially satisfied whenever $y_{t}$ has a degenerate distribution prior to some point. So it is satisfied in our case since $\mathbf{y}_{\mathbf{0}}, \mathbf{y}_{\mathbf{- 1}}, \ldots$. have degenerate distributions.

It is straightforward to introduce a time-invariant latent variable into these definitions. We shall say that "y does not couse $x$ conditional on a Latent vamiable c" if either

$$
\begin{aligned}
& x_{t+1} \text { is independent of } y_{1} \ldots ., y_{t} \text { conditionat on } \\
& x_{1}, \ldots, x_{t}, \text { o } \quad(t=1,2, \ldots I,
\end{aligned}
$$

or

$$
\begin{aligned}
& y_{t} \text { is independent of } x_{t+1}, x_{t+2}, \ldots \text { conditional on } \\
& x_{1}, \ldots, x_{t}, y_{1}, \ldots, y_{t-1}, 0 \text { (t=1,2,..); }
\end{aligned}
$$

they are equivalent. We shall say that "x is strictly exogenous conditional on a latent variable $e^{\prime \prime}$ if

$$
\begin{aligned}
& y_{t} \text { is independent of } x_{t+1}, x_{t+2}, \ldots \text { conditional on } \\
& x_{1}, \ldots, x_{t} \subset(t=1,2, \ldots) .
\end{aligned}
$$

Now let us return to the problem of distinguishing between serial

correlation and structural lagged dependent variables. Assume throughout

the discussion that $\mathbf{x}_{\mathbf{t}}$ and $\mathbf{y}_{\mathbf{t}}$ are not independent. We shall say that the relationship of $\mathbf{x}$ to $y$ is static if

$x$ is strictly exogenous and $y_{t}$ is independent of $x_{1}, \ldots, x_{t-1}$ conditional on $x_{t}$. 
Then I propose the following distinctions:

There is residual serial correlation if $y_{t}$ is not independent of $y_{1}, \ldots y_{t-1}$ conditionat on $x_{1}, \ldots, x_{t}$;

If the relationship of $\approx$ to $y$ is static, then there are no structural lagged dependent variables.

Suppose that $\mathbf{y}_{\mathbf{t}}$ and $\mathbf{x}_{\mathbf{t}}$ are binary and consider the probability that $\mathrm{y}_{2}=1$ conditional on $\left(\mathbf{x}_{1}, \mathbf{x}_{2}\right)=(0,0)$ and conditional on $\left(x_{1}, \mathbf{x}_{2}\right)=(1,0)$. Since $\mathbf{y}_{\mathbf{t}}$ and $\mathbf{x}_{\mathbf{t}}$ are assumed to be dependent, the distribution of $\mathbf{y}_{\mathbf{1}}$ is generally different in the two cases. If $y_{1}$ has a structural effect on $y_{2}$, then the conditional probability of $\boldsymbol{y}_{\mathbf{2}} \mathbf{=} 1$ should differ in the two cases, so that $\mathbf{y}_{2}$ is not independent of $\mathbf{x}_{1}$ conditional on $\mathbf{x}_{2}$.

Note:that this condition is one-sided: $\dot{I}$ am only offering a condition for there to be no structural effect of $y_{t-1}$ on $y_{t}$. There can be distributed lag relationships in which we would not want to say that $y_{t-1}$ has a structural effect on $\mathbf{y}_{\mathbf{t}}$. Consider the production function example with serial correlation in rainfall; assume for the moment that there is no variation in $c$. If the serial correlation in rainfall is not incorporated in the farmer's information set, then our definitions assert that there is residual serial correlation but no structural lagged dependent variables, since the relationship of $\mathbf{x}$ to $y$ is static. Now suppose that the farmer does use previous rainfall to predict future rainfall. Then the relationship of $\mathbf{x}$ to $\mathbf{y}$ is not static since $\mathbf{x}$ is not strictly exogenous. But we my not want to say that the relationship between $\mathbf{y}_{\mathbf{t}-1}$ and $\mathbf{y}_{\mathbf{t}}$ is structural, since the technology does not depend upon $\mathbf{y}_{\mathbf{t}-\mathbf{1}}$. 
How are these distinctions affected by latent variables? It should be clear that a time-invariant latent variable can produce residual serial correlation. A major theme of the paper has been that such a latent variable can also produce a failure of strict exogeneity. So consider conditional versions of these properties:

There is residual seriaz correlation conditional on a latent variable $c$ if $y_{t}$ is not independent of $y_{1}, \ldots, y_{t-1}$ conditionat on $x_{1}, \ldots, x_{t}, C$;

The retationship of $x$ to $y$ is static conditional on a latent voriable $c$ if $x$ is strictly exogenous conditional on $c$ and if $y_{t}$ is independent of $x_{1}, \ldots, x_{t-1}$ conditional on $x_{t}, \mathbf{c}$

If the relationship of $a$ to $y$ is static conditional on a latent variable $\boldsymbol{c}$, the there are no structural lagged dependent variabies.

A surprising feature of tiae linear predictor definition of strict exogeneity is that it is restrictive to assert that there exists some time-invariant latent variable $\mathrm{c}$ such that $\mathbf{x}$ is strictly exogenous conditional on $c$. This is no longer true when we use conditional independence to define strict exogeneity. For a counterexample, suppose that $\mathbf{x}_{\mathbf{t}}$ is a binary variable and consider the conditional strict exogeneity question, "Does there exist a time-invariant random variable $c$ such that $\mathbf{y}_{\mathbf{t}}$ is independent of $\mathbf{x}_{\mathbf{1}}, \ldots .$. xT conditional on $\mathbf{x}_{\mathbf{1}}, \ldots . . \mathbf{x}_{\mathbf{t}}$, $\mathbf{c}$ ?" The answer is "yes" since we can order the 2 ? possible outcomes of the binary

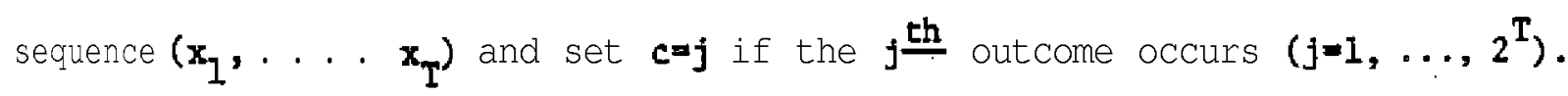
Now $\mathbf{y}_{t}$ is independent of $\mathbf{x}_{\mathbf{I}}, \ldots, \mathbf{x}_{\mathbf{T}}$ conditional on $\mathrm{c}$ !

For a nondegenerate counterexample, let $\mathbf{y}$ and $\boldsymbol{x}$ be binary random variables with 


$$
P\left(y=\alpha_{j}, x=\alpha_{k}\right)=\tau_{j k}>0, \sum_{j, k=1}^{2} \tau_{j k}=1,
$$

where $\alpha_{1}=1, \alpha_{2}=0$. Let $\gamma^{\prime}=\left(\tau_{11}, \tau_{12}, \tau_{21}, \tau_{22}\right)$. Then we can set

$$
\Upsilon=\sum_{m=1}^{4} \gamma_{m \sim m}^{e}, \gamma_{m}>0, \sum_{m=1}^{4} \gamma_{m}=1
$$

where $e_{\sim m}$ is a vector of zeros except for a one in the th component. Hence $\underset{\sim}{ }$ is in the interlot of the convex hull of $\{\underset{\sim \mathrm{m}}{\mathrm{e}}, \mathrm{m}=1, \ldots, 4\}$. Now consider the vector

$$
y(\delta, \lambda)=\left|\begin{array}{l}
\delta \lambda \\
\delta(1-\lambda) \\
(1-\delta) \lambda \\
(1-\delta)(1-\lambda)
\end{array}\right|
$$

The components if $\mathrm{y}(\delta, \lambda)$ give the probabilities $\mathrm{P}\left(\mathrm{y}=\alpha_{j}, \mathrm{x}=\alpha_{k}\right)$ when $\mathrm{y}$ and $\mathrm{x}$ are independent with $\mathrm{P}(\mathrm{y}=1)=\delta, \mathrm{P}(\mathrm{x}=1)=\lambda$. Set $e_{-\mathrm{m}}^{\mathrm{m}}=$ $\mathrm{Z}\left(\delta_{\mathrm{m}}, \lambda_{\mathrm{m}}\right)$ with $0<\delta_{\mathrm{m}}<1,0<\lambda_{\mathrm{m}}<1$. Then $Y$ will be in the interior of the convex hull of $\left\{\underset{\sim m}{e^{*}}, m=1, \ldots, 4\right\}$ if we choose $f_{m}, \lambda_{m}$ so that $e_{m}^{*}$ is sufficiently close to $\mathrm{e}_{\mathrm{m}}$. Hence

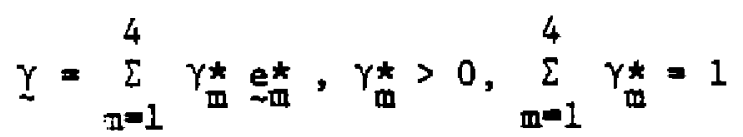

Let the components of $\stackrel{-}{\mathrm{m}}_{\mathrm{m}}^{\mathrm{k}}$ be $\left(\tau_{11}^{\mathrm{m}}, \tau_{12}^{\mathrm{m}}, \tau_{21}^{\mathrm{m}}, \tau_{22}^{\mathrm{m}}\right)$. Let $c$ be a random varlable with $P(c=m)=\gamma_{\text {m }}^{*}(m=1, \ldots, 4)$, and set

$$
P\left(y=\alpha_{j}, x=\alpha_{k} \mid c=m\right)=\tau_{f k}^{m} .
$$


Now $\mathrm{y}$ is independent of $\mathrm{x}$ conditional on $\mathrm{c}$, and the conditional distributions are nondegenerate.

If $\left(x_{1}, \ldots . x_{T}, y_{1}, \ldots, y_{T}\right)$ has a general multinomial distribution, then a straightforward extension of this argument shows that there exists a random variable $c$ such that $\left(y_{1}, \ldots . . y_{T}\right)$ is independent of $\left(x_{1}, \ldots . . x_{T}\right)$ conditional on $\mathbf{c}$, and the conditional distributions are nondegenerate.

A similar point applies to factor analysis. Consider a linear onefactor model. The specification is that there exists a latent variable $c$ such that the partial correlations between $\mathbf{y}_{\mathbf{1}}, \ldots . \mathbf{y}_{\mathbf{T}}$ are zero given $\mathbf{c}$. This is restrictive if $\mathrm{T}>3$. But we now know that it is not restrictive to assert that there exists a latent variable c such that $y_{1}, \ldots . . y_{T}$ are independent conditional on $c$.

It follows that we cannot test for conditional strict exogeneity without imposing functional form restrictions; nor can we test for a conditionally static relationship without restricting the functional forms.

This point is intimately related to the fundamental difficulties created by incidental parameters in nonlinear models. The labor force participation example is assumed to be static conditional on $\mathrm{c}$. We shall present some tests of this in Section 5, but we shall be jointly testing that proposition and the functional forms - a truly nonparametric test cannot exist. We stressed in the probit model that the specification for the distribution of $\mathrm{c}$ conditional on $\mathbf{x}$ is restrictive; we avoided such a restrictive specification in the logit model but only by imposing a restrictive functional form on the distribution of $y$ conditional on $\mathbf{x}, \mathrm{c}$. 


\subsection{Duration lodets}

In many problems the basic data is the amount of tine spent in a

state. For example, a complete description of an individual's labor

force participation history is the duration of the first spell of parti-

cipation and the date it began, the duration of the following spell of non-

participation, and so on. This complete history will generate a binary

sequence when it is cut up into fixed length periods, but these periods

may have little to do with the underlying process. 24

In particular, the measurement of serial correlation depends upon the period of observation. As the period becomes shorter, the probability that a person who worked last period will work this period approaches one. So finding significant serial correlation may say very iftele about the underlying process. Or consider a spell that begins near the end of-a period; then it is likely to overlap into the next period, so that previous employment raises the probability of current employment.

Consider the underlying process of time spent in one state followed by time spent in the other state. If the individual's history does not help to predict his future given his current stace, then this is a Markov process. Whereas serial independence in continuous time has the absurd Implication that mean duration of a spell is zero, the Markov property does provide a fruitful starting point. It has two Implications: the individual's history prior to the current spell should not affect the distribution of the length of the current spell; and the amount of time spent in the current state should not affect the distribution of remaining time in that state.

So the first requirement of the Markov property is that durations of the spells be independent of each other. Assuming stationarity, this 
implies an alternating renewal process. The second requirement is that the distribution of duration be exponential, so that we have an alternating Poisson process. We shall refer to departures from this model as duration dependence.

A test of this Markov property using binary sequences will depend upon what sampling scheme is being used. The simplest case is point sampling, where each period we determine the individual's state at a particular point in time, such as July 1 of each year. Then if an individual is following an alternating Poisson process, her history prior to that point is irrelevant in predicting her state at the next interview. So the binary sequence generated by point sampling should be a Markov chain.

It is possible to test this in a fixed effects model that allows each individual to have her own two exponential rate parameters $\left(c_{i 1}, c_{12}\right)$ in the alternating Poisson process. The idea is related to the conditional likelihood approach in the fixed effects logit model. Let $\mathbf{s}_{i j k}$ be the number of times that individual 1 is observed making a transition from state $\mathbf{j}$ to state $k(\mathbf{j}, k=\mathbf{m}, \mathbf{2})$. Then the initial state and these four transition counts are sufficient statistics for the Markov chain. Sequences with the same initial state and the same transition counts should be equally likely. This is the Markov form of de Finett's (1975) partial exchangeabllity. 25 So we can test whether the Markov property holds conditional on $\boldsymbol{c}_{\mathbf{1 1}}, \boldsymbol{c}_{\mathbf{1} 2}$ by testing whether there is significant variation in the sample frequencies of sequences with the same transition counts.

This analysis is relevant if, for example, each year the survey question is "Did you have a job on July 1?" In the Michigan Panel Study of Income Dynamics, however, the most commonly used question 'for generating participation sequences is "Did your wife do any work for money last year?" This interval sampling leads to a more complex analysis, since even if the individual is following an alternating Poisson process, the binary sequence 
generated by this sampling scheme is not a Markov chain. suppose that $y_{t-1}=1$, so that we know that the individual worked at some point during the previous period. What is relevant, however, is the individual's state at the end of the period, and $y_{t-2}$ will affect the probability that the spell of work occurred early in period t-l instead of late in the period.

Nevertheless, it is possible to test whether the underlying process is alternating Poisson. The reason is that if $\mathbf{y}_{\mathbf{t - 1}}=0$, we know that the individual never worked during period $t-l$, and so we know the state at the end of that period; hence $\mathbf{y}_{\mathbf{t}-\mathbf{2}}, \mathbf{y}_{\mathbf{t}-\mathbf{3}}, \cdot$. . are irrelevant. So we have

$$
\begin{aligned}
& P\left(y_{t}=1 \text { lic } 1, c_{2}, y_{t-1}, y_{t-2}, \ldots\right) \\
& \left.\quad \therefore N Y,=1 \mid c_{1}, c_{2}, y_{t-1}=\ldots,=y_{t-d}=1, y_{t-d-1}=0\right) \\
& \quad=P\left(y_{t}=1 \mid c_{1}, c_{2}, d\right),
\end{aligned}
$$

where $d$ is the number of consecutive preceding periods that the individual was in state 1 .

Let $\mathbf{s}_{01}$ be the number of times in the sequence that 1 is preceded by 0 ; let $\mathbf{s}_{011}$ be the number of times that 1 is preceded by 0,1 ; etc. Then sufficient statistics are $\mathrm{s}_{01}, \mathbf{s}_{011}, \ldots$, as well as the number of consecutive ones at the beginning $\left(n_{1}\right)$ and at the end $\left(n_{T}\right)$ of a sequence. 26 For an example with $\mathbf{T}=5$; let $\mathbf{n}_{1}=0, \mathbf{n}_{5}=0, \mathbf{s}_{01}=1, \mathbf{s}_{011}=1$, $\mathbf{s}_{0111}=\ldots \times 0$; then we have $P(0,1,1,0,0 \mid c)$ $\left.=\mathrm{NY}_{1}=0 \mid \mathrm{c}\right) \mathrm{P}(1 \mid 0, \underset{\sim}{\mathrm{c}}) \mathrm{P}(1 \mid 0,1, \underset{\sim}{\mathrm{c}}) \mathrm{P}(0 \mid 0,1, \mathrm{I}, \underset{\sim}{\mathrm{c}}) \mathrm{P}(0 \mid 0, \underset{\sim}{\mathrm{c}})$ $P(0,0,1,1,0 \mid c)$

$=P\left(y_{1}=0 \mid c\right) P(0 \mid 0, c) P(1 \mid 0, c) P(1 \mid 0,1, c) P(0 \mid 0,1,1, c)$ 
where $c=\left(c_{1}, c_{2}\right)$. Thus these two sequences are equally likely conditional

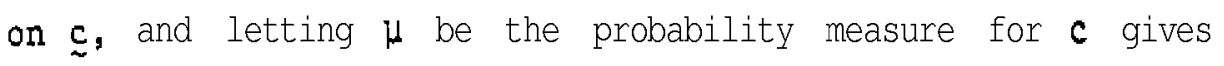

$$
\begin{aligned}
& P(0,1,1,0,0)=I P(0,1,1,0,0 \mid \underset{\sim}{c}) \mu\left(d_{\sim}^{c}\right) \\
& =\int P(0,0,1,1,0 \mid c) \mu(d \underset{\sim}{c})=P(0,0,1,1,0)
\end{aligned}
$$

So the alternating Poisson process implies restrictions on the multinomial distribution for the binary sequence.

These tests are indirect. The duration dependence question is clearly easier to answer using surveys that measure durations of spells. Such duration data raises a number of new econometric problems, but we shall not pursue them here. ${ }^{27}$ I would simply like to make one connection with the methods that we have been discussing.

Let us simplify to a one state process; for example, $\mathbf{y}_{\mathbf{1 t}}$ can be $\mathbf{t}$ :e duration of the time interval between the starting date of the $\mathbf{1}$ th individual's th job and his $(t+1) \stackrel{\text { th }}{=}$ job. Suppose that we observe $T>1$ jobs for each of the $N$ individuals, a not innocuous assumption. Impose, the restriction that $\boldsymbol{y}_{\mathbf{i t}}>0$ by using the following specification:

$$
\begin{aligned}
& y_{i t}=\exp \left(\beta x_{i t}+c_{i}+u_{i t}\right), \\
& E^{*}\left(u_{i t} \mid x_{i}\right)=0(t-1, \ldots, T)
\end{aligned}
$$

where $x_{i}^{\prime}=\left(x_{i 1}, \ldots x_{i T}\right)$. Then

$$
E^{*}\left(\ln y_{i t} \mid x_{1}\right)=\beta x_{1 t}+\lambda^{\prime} x_{1}
$$

and our Section 2 analysis applies. The strict exogeneity assumption has 
a surprising implication in this context. Suppose that $\mathbf{x}_{\mathbf{i t}}$ is the

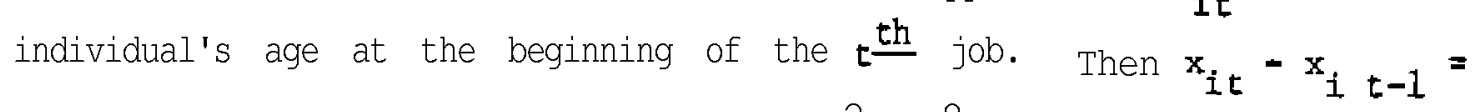
$y_{i, t-1}^{--}$age is not strictly exogenous. ${ }^{2} 8$

\section{INEERENCE}

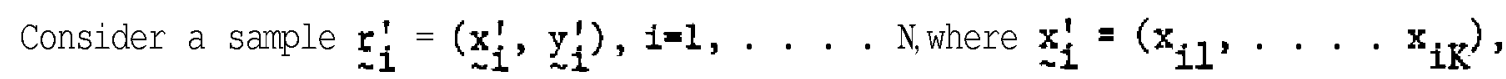
$\mathbf{y}_{\mathbf{i}}^{\prime}=\left(\mathbf{y}_{\mathbf{i l}}, \ldots . \mathbf{y}_{\mathbf{i M}}\right)$. We shall assume that $\mathbf{r}_{\mathbf{i}}$ is independent and identically distributed (1.i.d.) according to some multivariate distribution with finite fourth moments and $\mathbf{E}\left(\mathbf{x}_{\mathbf{i}} \mathbf{x}_{i}^{\prime}\right)$ nonsingular. Consider the minimum mean-square error linear predictors, 29

$$
E^{*}\left(y_{i m} \mid x_{i}\right)=\pi_{-1}^{\prime} x_{i} \quad(m=1, \ldots M),
$$

which we can write as

$$
E^{\star}\left(y_{i} \mid x_{i}\right)=\underset{\sim}{\pi} x_{i}, \quad \underline{I}=E\left(\underline{y}_{i} x_{i}^{\prime}\right)\left[E\left(\underline{\sim}_{i} x_{i}^{\prime}\right)\right]^{-1}
$$

We want to estimate $\Pi$ subject to restrictions and to test those restrictions. For example, we may want to test whether a submatrix of II has the form $B I+\sum_{2}^{A}$.

We shall not assume that the regression function $\mathbf{E}\left({\underset{\sim}{\mathbf{1}}}_{\mathbf{1}} \mid \underline{\mathbf{x}}_{\mathbf{1}}\right)$ is linear. For although $\mathbf{E}\left(\underline{\sim}_{\mathbf{i}} \mid \mathbf{x}_{\mathbf{i}}, \boldsymbol{c}_{\mathbf{i}}\right)$ may be linear (indeed, we hope that it is), there is generally no reason to insist that $\mathbf{E}\left(\mathbf{c}_{\mathbf{i}}\left(\mathbf{x}_{\mathbf{i}}\right)\right.$ is linear. So we shall present a theory of inference for linear predictors. Furthermore, even if the regression function is linear, there may be heteroskedasticity -- due

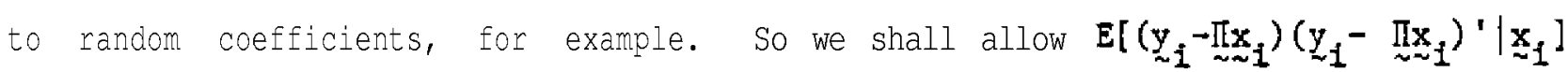
to be an arbitrary function of $\underline{\mathbf{x}}_{1}$ 


\subsection{The Estimation of Linear Eredictors}

Let $\mathbb{w}_{i}$ be the vector formed from the distinct elements of $\underline{\mathbf{r}}_{\mathbf{i}} \mathbf{r}_{\mathbf{1}}^{\prime}$

that have nonzero variance. 30 Since $\underset{\sim i}{\mathbf{r}}=\left(\mathbf{x}_{\mathbf{i}}^{\prime}, \mathbf{y}_{\mathbf{i}}^{\prime}\right)$ is i.i.d., it follows

that $\mathbf{w}_{\boldsymbol{i}}$ is i.i.d. This simple observation is the key to our results. Since $\underset{\sim}{I}$ is a function of $\underset{\mathbf{E}}{\left(\mathbf{w}_{\mathbf{i}}\right)}$, our problem is to make inferences about a function

of a population mean, under random sampling.

Let $\boldsymbol{\mu}=\mathbf{E}\left(\boldsymbol{w}_{\mathbf{1}}\right)$ and let $\boldsymbol{\pi}$ be the vector formed from the columns of $\boldsymbol{I}_{\sim}$

$\left(a=\operatorname{vec}\left(\Pi_{\sim}^{\prime}\right)\right)$. Then $\pi_{\sim}$ is a function of $\underset{\sim}{\mu}: \pi_{\sim}=\underset{\sim}{h}(\underset{\sim}{\mu})$. Let $\underset{\sim}{\bar{w}}=\Sigma_{1=1}^{\mathbb{N}} \underset{\sim 1}{\mathbb{w}} / \mathbb{N}$;

then $\hat{\pi} \boldsymbol{\sim} \mathbf{h}(\bar{w})$ is the least squares estimator:

$$
\hat{\pi}=\operatorname{vec}\left[\left(\sum_{i=1}^{N} x_{-i}^{N} y_{i}^{\prime} \sum_{i=1}^{-1} \sum_{i=1}^{N} x_{i}^{\prime}\right]\right. \text {. }
$$

By the strong law of large numbers, $\overline{\boldsymbol{w}}$ converges almost surely to $\mu^{\circ}$ as $N+\infty$ $\left(\overrightarrow{\mathbf{w}} \stackrel{\mathbf{a}, \mathbf{s} .}{\longrightarrow} \mu^{0}\right)$, where $\mu^{0}$ is the true value of $\mu^{\circ}$. Let $\pi^{0}=\mathbf{h}\left(\mu^{0}\right)$. Since

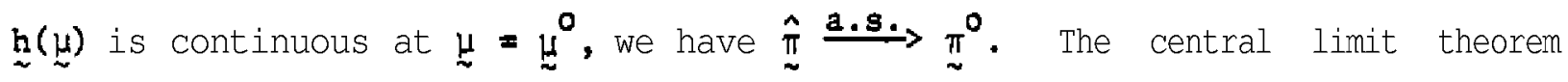
implies that

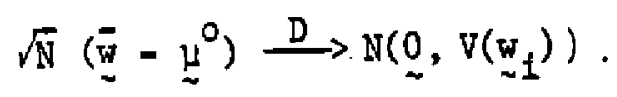

Since $\underline{\boldsymbol{h}}(\boldsymbol{\mu})$ is differentiable at $\boldsymbol{\mu}_{\boldsymbol{\omega}}=\underline{\mu}^{0}$, the $\boldsymbol{\delta}$-method gives

$$
\sqrt{\mathrm{N}}\left(\underset{\pi}{\pi}-\pi^{0}\right) \stackrel{\mathrm{D}}{\longrightarrow} \mathrm{N}(\underline{0}, \Omega)
$$

where

$$
\Omega=\frac{\partial h\left(\mu^{0}\right)}{\partial \mu^{\dagger}} \cdot v(\underset{\sim}{w}) \frac{\partial h^{\prime}\left(\mu^{0}\right)}{\partial \mu} \cdot 31
$$

We have derived the limiting distribution of the least squares estimator.

This approach was used by cramer (1946) to obtain limiting normal distributions

for sample correlation and regression coefficients (p. 367); he presents an 
explicit formula for the variance of the limiting distribution of a sample correlation coefficient ( p. 359). Kendall and Stuart (1961, p. 293) and Goldberger (1974)' present the formula for the variance of the limiting distribution of a simple regression coefficient.

Evaluating the partial derivaives in the formula for $\Omega$ is tedious.

That calculation can be simplified since $\hat{\pi}$ has a "ratio" form. In the case of simple regression with a zero incercept, we have $\pi=E\left(y_{i} x_{i}\right) / E\left(x_{i}^{2}\right)$ and

$$
\sqrt{N}\left(\hat{\pi}-\pi^{0}\right)=\left(\sum_{i=1}^{N} y_{1} x_{i}-\pi^{0} \sum_{i-1}^{N} x_{i}^{2}\right) /\left[\sqrt{N}\left(\sum_{i=1}^{N} x_{i}^{2} / N\right)\right] .
$$

Since $\Sigma_{\mathbf{1}=1}^{\mathbf{N}} \mathbf{x}_{\mathbf{i}}^{2} / \mathbf{N} \stackrel{\mathbf{a} \cdot \mathbf{s} .}{\longrightarrow} \mathbf{E}\left(\mathbf{x}_{\mathbf{i}}^{2}\right)$, we obtain the same limiting distribution by working with

$$
\sum_{i=1}^{N}\left[\left(y_{i}-\pi^{\circ} x_{i}\right) x_{i}\right] /\left[\sqrt{N} \Xi\left(x_{i}^{2}\right)\right]
$$

The definition of $\pi^{0}$ gives $E\left[\left(\mathbf{y}_{1}-:^{o} \mathbf{x}_{1}\right) \mathbf{x}_{1}\right]=0$, and so the central limit theorem implies that

$$
\sqrt{\mathbb{N}}\left(\hat{\pi}-\pi^{0}\right) \stackrel{D}{\longrightarrow} \mathbb{N}\left[0, E\left[\left(y_{i}-\pi^{o} x_{i}\right)^{2} x_{i}^{2}\right] /\left[E\left(x_{i}^{2}\right)\right]^{2}\right\}
$$

This approach was used by white (1980) to obtain the limiting distribution for univariate regression coefficients. ${ }^{32}$ In the Appendix (Proposition 7) we follow White's approach to obtain

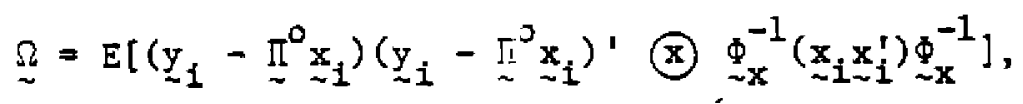

where $\underset{\sim \mathbf{x}}{\Phi} \mathbf{=} \boldsymbol{E}\left({\underset{\sim}{1} \mathbf{x}}_{\sim} \mathbf{x}_{1}^{\prime}\right)$. A consistent estimator of $\boldsymbol{\Omega}$ is readily available from the corresponding sample moments: 
$(4.2)$

$$
\begin{aligned}
& \hat{\partial}=\frac{1}{N} \sum_{i=1}^{N}\left[\left(y_{i}-\hat{I} x_{i}\right)\left(y_{i}-\hat{\Pi} \underset{\sim}{x_{i}}\right)^{\prime}(x) \underset{\sim x}{s}\left(x_{i} x_{i}^{\prime}\right) \underset{\sim x}{s}{ }^{-1}\right] \\
& \stackrel{\text { a.s. }}{\longrightarrow} \Omega \text {, }
\end{aligned}
$$

where $\quad S_{-\mathrm{x}}=\Sigma_{i=1}^{\mathrm{N}}{\underset{\sim}{i} \mathrm{i}}_{\mathbf{i}}^{\prime} / \mathrm{N}$.

If $\mathbb{E}(\underbrace{}_{i} \mid{\underset{\sim}{i}}_{i})=\underset{\sim}{\mathbf{x}_{i}}$, so that the regression function is linear, than

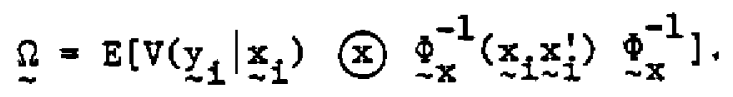

If $V\left({\underset{\sim}{i}}_{i} \mid \mathbf{x}_{i}\right)$ is uncorrelated with $\underset{i}{z_{i}} \mathbf{x}_{i}^{\prime}$, then

$$
\Omega=E\left[V(\underbrace{}_{i} \mid x_{i})\right] \circledast \underset{\sim x}{\Phi}
$$

If the conditional variance is homoskedastic, so that $v\left({\underset{\sim}{i}}_{i} \mid \underset{\sim}{x_{i}}\right)=\underset{\sim}{\Sigma}$ does

not depend on $\mathbf{x}_{\mathbf{1}}$, then

$$
\Omega=\Sigma \otimes \underset{\sim}{\sim} \Phi_{x}^{-1}
$$

\subsection{Imposing Restrictions: The Minimom Distance Estimator}

Since $\underset{\sim}{\Pi}$ is a function of $\mathbf{E}\left({\underset{\sim}{\mathbf{i}}}_{\mathbf{i}}\right)$, restrictions on $\mathbb{I}$ imply restrictions

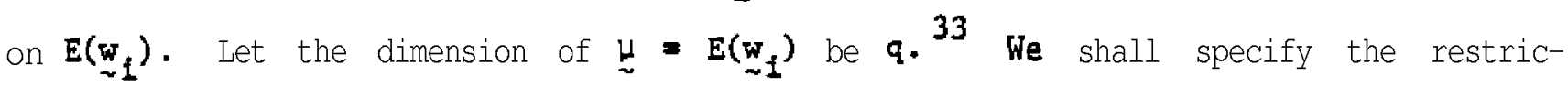
tions by the condition that $\mu$ depends only on a $\mathbf{p}^{\times 1}$ vector $\theta$ of unknown

parameters: $\underline{\Sigma}=\mathbf{g}(\theta)$, where $g$ is a known function and $p \leq \mathbf{q}$. The domain of $\theta$ is $\mathrm{T}$, a subset of $\mathrm{p}$-dimensional Euclidean space $\left(\mathbf{R}^{\mathbf{P}}\right)$ that contains the true value $\theta^{\circ}$. So the restrictions Imply that $\stackrel{\sim}{\sim}^{\circ}=\mathbf{g}\left(\theta^{\circ}\right)$ is confined to a certain subset of $\mathbf{R}^{q}$. 
We can impose the restrictions by using a minimum distance estimator: choose $\hat{\theta}$ to

$$
\left.\min _{\theta \varepsilon T} \sum_{i=1}^{N}{\underset{\sim}{i}}_{i} \underset{\sim}{g(\theta)}\right]^{\prime}{\underset{\sim N}{A N}}_{\sim}\left[w_{i}-\underset{\sim}{g}(\theta)\right],
$$

where $\underset{\sim N}{A_{N}} \stackrel{\text { a.s. }}{\longrightarrow} \underset{\sim}{ }$ and $\Psi$ is positive definite. ${ }^{34}$ This minimization problem is equivalent to the following one: choose $\hat{\theta}$ to

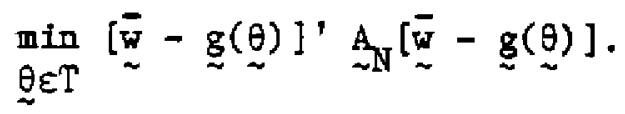

The properties of $\hat{\theta}$ are developed, for example, in Malinvaud (1970, Chap. 9). Since $g$ does not depend on any exogenous variables, the derivation of these properties can be simplified considerably, as in Chiang (1956: and Ferguson $(1958) \cdot 35$

For completeness, we shall state a set of regularity concitions and the properties that they imply:

Assumption 1. $\underset{\sim \mathrm{N}}{\mathbf{a} . \mathbf{s} .} \underset{\mathrm{g}}{\mathbf{g}}\left(\underset{\sim}{\left(\theta^{\circ}\right.}\right)$; $\mathrm{T}$ is a compact subset of $\mathbb{R}^{\mathrm{P}}$ that contains $\underline{\theta}^{\circ} ; g$ is continuous on $\mathrm{T}$, and $\mathbf{g}(\theta)=\mathbf{g}\left(\underline{\theta}^{\circ}\right)$ for $\theta \in \mathrm{T}$ implies that $\theta \mathbf{=} \underline{\theta}^{\circ}$; A $\stackrel{\text { a.s... }}{\longrightarrow} \underset{\sim}{\Psi}$, where $Y$ is positive definite.

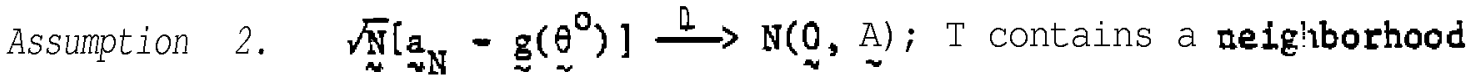
$\bar{\Xi}_{0}$ of $\underline{\theta}^{\circ}$ in which $g$ has continuous second partial derivatives; rank $(G)=$ p, where $G=\partial g\left(\theta^{\circ}\right) / \partial \theta^{\prime}$.

Choose $\hat{\theta}$ to

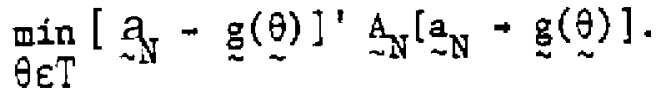


Enoposition I. If Assumption 1 is satisfied, then $\hat{\epsilon} \stackrel{\text { a.s. }}{\rightarrow} z^{0}$.

Enoposition 2. If assumptions 1 and 2 are satisfied, then $\sqrt{N}\left(\theta_{\sim}^{\hat{\alpha}}-\underset{\sim}{\theta^{\circ}}\right) \stackrel{D}{\longrightarrow} N(\underset{\sim}{0}, \underset{\sim}{\Lambda})$, where

$$
\left.\left.\underset{\sim}{A}={\underset{\sim}{(G}}_{\sim^{\prime}}^{\Psi}\right)^{-1} G^{\prime} \underset{\sim}{\Psi} \underset{\sim}{\Psi} \underset{\sim}{\Psi} \underset{\sim}{G} G^{\prime} \Psi G\right)-1
$$

If $A$ is positive definite, then $A-\left(\stackrel{\sim}{G}^{\prime} \Delta^{-1} G\right)^{-1}$ is positive semi-definite; hence an optimal choice for $\Psi$ is $\underset{\sim}{\mathbf{A}}$.

Proposition 3. If Assumptions 1 and 2 are satisfied, if $\Delta$ is a qxq positivedefinite matrix, and if $\underset{\sim}{\mathbb{N}} \stackrel{\text { a.s.s. }}{\rightarrow} \Delta^{-1}$, then

$$
\mathbb{N}[\underset{\sim N}{a}-g(\hat{\theta})]^{\prime} \underset{\sim N}{A}\left[\underset{\sim N}{g}-\underset{\sim}{g}(\underset{\sim}{\theta}) 1 \stackrel{D}{\rightarrow} x^{2}(q-p)\right.
$$

Now consider imposing additional restrictions, which are expressed by the condition that $\underset{\sim}{\theta}=\underline{\underline{f}}(\alpha)$, where $\underset{\sim}{\alpha}$ is $s^{\times}{ }^{\prime}(s \leq p)$. The domain of $\alpha$ is $\mathrm{T}_{1}$, a subset of $R^{\mathbf{s}}$ that contains the true value $\alpha^{0}$. so $\underline{\theta}^{0}=\underset{\sim}{\mathbf{f}}\left(\alpha^{0}\right)$ is confined to a certain subset of $\mathbf{R}^{\mathbf{P}}$.

Assumption 2'. $\mathrm{T}_{1}$ is a compact subset of $\mathrm{R}^{\mathbf{s}}$ that contains $\underline{q}^{0}$; $f$ is a continuous mapping from $T_{1}$ into $T ; f(\alpha)=\theta^{\circ}$ for $\alpha \in T_{1}$ implies $\alpha_{\alpha}=\alpha^{\circ}$;

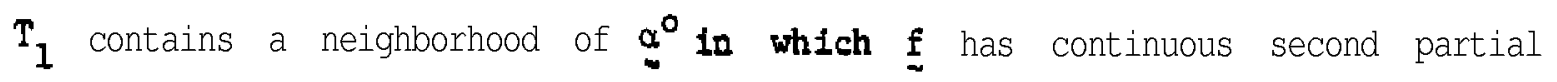
derivatives; rank $(F)=s$, where $F=\partial f\left(\alpha^{\circ}\right) / \partial \alpha$.

Let $h(\underset{\sim}{\alpha})=g[\underset{\sim}{f}(\underset{\sim}{\alpha})]$. Choose $\hat{\alpha}$ to

$$
\min _{\alpha \in \mathrm{T}_{1}}\left[a_{\mathrm{N}}-\underset{\alpha}{\mathrm{h}}(\alpha)\right]^{\prime}{\underset{\sim}{\mathrm{N}}}_{\mathrm{N}}\left[\mathrm{a}_{\mathrm{N}}-\underset{\mathrm{h}}{\mathrm{h}}(\underline{\alpha})\right]
$$


Proposition $3^{\prime}$. If Assumptions 1, 2, and $2^{\prime}$ are satisfied, if $\Delta$ ispositive definite, and if $\underset{\sim}{A} \stackrel{\text { a.s. }}{\longrightarrow} \Delta^{-1}$, then $d_{1}-d_{2} \stackrel{\mathbb{D}}{\longrightarrow} x^{2}(p-s)$, where

$$
\begin{aligned}
& d_{I}=N\left[a_{\sim N}-\underset{\sim}{h}(\hat{\alpha})\right]^{\prime}{\underset{\sim N}{A}}_{\sim N}\left[a_{N}-\underset{\sim}{h}(\hat{\alpha})\right], \\
& d_{2}=\mathbb{N}\left[\underset{\sim}{a} \mathbb{N}-g(\hat{\theta}) I^{\prime} A_{\sim \mathbb{N}}\left[a_{\sim}-g(\hat{\theta})\right] .\right.
\end{aligned}
$$

Furthermore, $d_{1}-d_{2}$ is independent of $d_{2}$ in their limiting joint distribution.

Suppose that the restrictions involve only $\pi$. We specify the restrictions by the condition that $\pi=\underset{\sim}{f} \underset{\sim}{(6)}$, where $\underset{\sim}{\delta}$ is $\mathbf{s} \times \mathbf{I}$ and the domain of 6 is $T_{1}$, a subset of $\mathbf{R}^{\mathbf{s}}$ that includes the true value $\delta^{\circ}$. Consider the following estimator of $\underset{\sim}{\delta^{\circ}}$ : choose $\underset{\sim}{\hat{\delta}}$ to

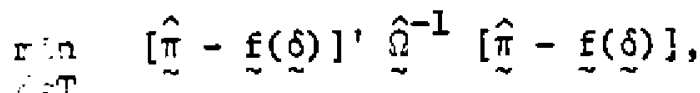

$$
\begin{aligned}
& \mathrm{T}_{1}
\end{aligned}
$$

where $\hat{\Omega}$ is : iven in (4.2), and we assume that $\stackrel{\Omega}{\sim}$ in $(4.1)$ is

positive definite. If $\mathrm{Tl}$ and $\underset{\sim}{f}$ satisfy assumptions 1 and 2 , then $\hat{\delta} \stackrel{\text { a.s.s. }}{\longrightarrow} \stackrel{\sim}{\circ}^{\circ}$,

$$
\sqrt{\mathbb{N}}(\hat{\delta}_{\sim}^{\hat{\delta}}-\underbrace{0}) \stackrel{D}{\longrightarrow} \mathbb{N}\left(\underset{\sim}{0},\left[\underline{F}_{-}^{\prime} \Omega^{-1} \underset{\sim}{F}\right]^{-1}\right),
$$

and.

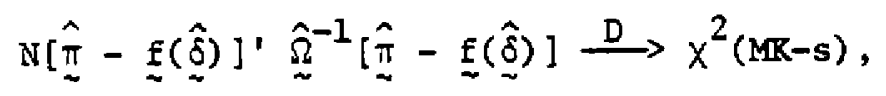

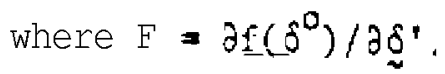

We can also estimate $\stackrel{\delta}{\sim}^{\circ}$ by applying the minimum distance procedure to $\boldsymbol{w}$ instead of to $\hat{\pi}$. Suppose that the components of ${\underset{\sim}{\mathcal{i}}}_{\text {are }}$ arranged so that

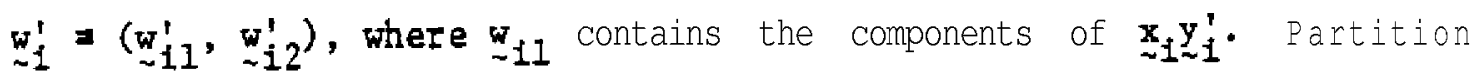

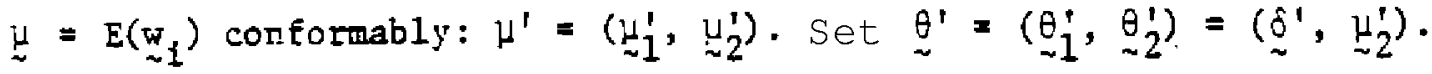


Assume that $\mathrm{v}\left(\mathrm{w}_{i}\right)$ is positive definite. Now choose $\hat{\theta}$ to

$$
\min _{\theta \varepsilon T}[\bar{w}-\underset{\sim}{g}(\theta)]^{\prime}{\underset{\sim}{N}}_{N}[\bar{w}-\underset{\sim}{g}(\theta)]
$$

where

$$
\stackrel{\text { a.s. }}{\rightarrow} \mathrm{V}^{-1}\left(\mathrm{w}_{\mathrm{i}}\right)
$$

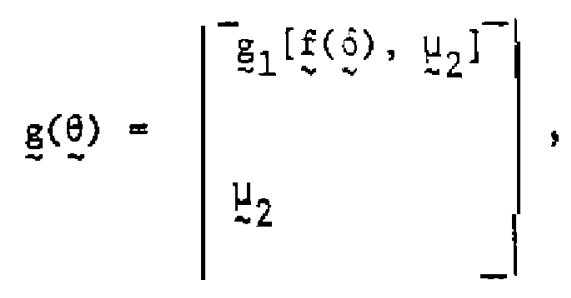

and $g_{1}(\underset{\sim}{\pi}, \underset{\sim}{\mu})={\underset{\sim}{\mu}}_{1}$ Then $\hat{\theta}_{\sim}$ gives an estimator of $\delta^{\circ}$; it has the same limiting distribution as the estimator $\underset{\sim}{\sim}$ that we obtained by applying the minimum distance procedure to $\hat{\pi} .36$

This framework leads to some surprising results on efficient estimation. For a simple example, we shall use a univariate linear predictor model,

$$
E^{*}\left(y_{i} \mid x_{11}, x_{12}\right)=\pi_{0}+\pi_{1} x_{i 1}+\pi_{2} x_{12}
$$

Consider imposing the restriction $\pi_{\mathbf{2}} \boldsymbol{=} 0$. Then the conventional estimator of $\pi_{1}$ is $b_{\mathbf{y x}_{1}}$, the slope coefficient in the least squares regression of $y$ on $\mathbf{x}_{\mathbf{l}}$. We shall show that this estimator is generally less efficient than the minimum distance estimator if the regression function is nonlinear or if there is hetaroskedasticity.

Let $\hat{\pi}_{1}, \hat{\pi}_{\mathbf{2}}$ be the slope coefficients in the least squares multiple regression of $y$ on $\mathbf{x}_{1}, \mathbf{x}_{2}$. The uinimum distance estimator of $\pi_{1}$ under the restriction $\pi_{2}=0$ can be obtained as $\hat{\sigma}=\hat{\pi}_{1}+\tau \hat{\pi}_{2}$, where $\tau$ is chosen to iuinimize the (estimated) variance of the limiting distribution of $\hat{\delta}$; this gives 


$$
\hat{\delta}=\hat{\pi}_{1}-\frac{\hat{\omega}_{12}}{\hat{\omega}_{22}} \hat{\pi}_{2},
$$

where $\hat{\omega}_{j k}$ is the estimated covariance between $\hat{\pi}_{j}$ and $\hat{\pi}_{k}$ in their Limiting distribution. Since $\hat{\pi}_{1}=b_{y_{1}}=\hat{\pi}_{2} b_{x_{2} x_{1}}$, we have

$$
\hat{\delta}=b_{y x_{1}}-\left(b_{x_{2} x_{1}}+\frac{\hat{\omega}_{12}}{\hat{\omega}_{22}}\right) \hat{\pi}_{2} .
$$

If $E\left(y_{i} \mid x_{i 1}, x_{i 2}\right)$ is linear and if $V\left(y_{i} \mid x_{i 1}, x_{i 2}\right)=\sigma^{2}$, then $\omega_{12} / \omega_{22} \mathbf{m}$ $-\operatorname{Cov}\left(\mathbf{x}_{i 1}, \mathbf{x}_{i 2}\right) / v\left(x_{i 1}\right)$ and $\hat{\delta}=b_{y x_{1}}$. But in general $\hat{\delta} \neq b_{y x_{1}}$ and $\hat{\delta}$ is more efficient than $b_{y_{1}}$. The source of the efficiency gain is that the limiting distribution of $\hat{\pi}_{2}$ has a zero mean (if $\pi_{2}=0$ ), and so we can reduce variance without introducing any bias if $\hat{\pi}_{2}$ is correlated with $b_{\mathbf{y x}}$. Under the assumptions of linear regression and homoskedasticity, $b_{\mathbf{y} \mathbf{x}_{\mathbf{1}}}$ and $\hat{\pi}_{\mathbf{2}}$ are uncorrelated; but this need not be true in the more general framework that we are using.

\subsection{Simultaneous Equations: A Generalization of Two-and Three- Stage Least Squores}

Given the discussion on imposing restrictions, it is not surprising that two-stage least squares is not, in general, an efficient procedure for combining instrumental variables. I shall demonstrate this with a simple example. Assume that $\left(\mathbf{y}_{\mathbf{1}}, \mathbf{z}_{\mathbf{1}}, \mathbf{x}_{\mathbf{1 1}}, \mathbf{x}_{\mathbf{i} 2}\right)$ is $\mathbf{1 . i . d .}$ according to some distribution with finite fourth moments, and that

$$
y_{1}=\delta z_{1}+v_{i}
$$

where $\mathbf{E}\left(\mathbf{v}_{\mathbf{i}} \mathbf{x}_{\mathbf{i} 1}\right)=\mathbf{E}\left(\mathbf{v}_{\mathbf{i}} \mathbf{x}_{\mathbf{i} 2}\right)=0$. Assume also that $\mathbf{E}\left(\mathbf{z}_{\mathbf{i}} \mathbf{x}_{\mathbf{i} 1}\right) \neq 0, \mathbf{E}\left(\mathbf{z}_{\mathbf{i}} \mathbf{x}_{ \pm 2}\right) \neq 0$. 
Then there are two instrumental variable estimators that both converge a.s. to $\delta$ :

$$
\begin{aligned}
& \hat{\delta}_{j}=\sum_{i=1}^{N} y_{i} x_{i j} / \sum_{i=1}^{N} z_{i} x_{i j} \\
& \sqrt{N}\left\{\left(\begin{array}{l}
\hat{\delta}_{i} \\
\hat{\delta}_{2}
\end{array}\right)-\left(\begin{array}{c}
\delta \\
\delta
\end{array}\right)\right\} \stackrel{D}{\longrightarrow} \mathbb{N}(0, \Lambda),
\end{aligned}
$$

where the j, $k$ element of $\Lambda$ is

$$
\lambda_{j k}=\frac{E\left[\left(y_{i}-\delta z_{i}\right)^{2} x_{i j} x_{i k}\right]}{E\left(z_{i} x_{i j}\right) E\left(z_{i} x_{i k}\right)} \quad(j, k=1,2) .
$$

The two-stage 1...ast squares estimator combines $\hat{\delta}_{1}$ and $\hat{\delta}_{\text {, by forming }}$ $\hat{\mathbf{z}}_{\mathbf{i}}=\dot{\pi}_{\mathbf{1}} \mathbf{x}_{\mathbf{i}}+\hat{\pi}_{\mathbf{2}} \mathbf{x}_{\mathbf{i} 2}$, ' ased on the least squares regression of $\mathbf{z}$ on $\mathbf{x}_{\mathbf{1}}$, $\mathrm{x} 2$ (assume that $E\left[\left(\mathbf{x}_{11}, \mathbf{x}_{12}\right)^{\prime}\left(\mathbf{x}_{11}, \mathbf{x}_{12}\right)\right]$ is nonsingular):

$$
\hat{\delta}_{\text {TSLS }}=\sum_{1=1}^{\hat{H}} \mathrm{y}_{i} \hat{z}_{i} / \sum_{i=1}^{N} z_{i} \hat{z}_{i}=\hat{\alpha} \hat{\delta}_{1}+(1-\hat{a}) \hat{\delta}_{2} \text {, }
$$

where

$$
\hat{\alpha}=\hat{\pi}_{I} \sum_{i=1}^{N} z_{i} x_{11} /\left(\pi_{1} \sum_{i=1}^{N} z_{i} x_{i 1}+\hat{\pi}_{2} \sum_{i=1}^{N} z_{i} x_{12}\right) .
$$

Since $\hat{\alpha} \stackrel{\text { a.s. }}{\longrightarrow} \alpha, \sqrt{\mathbb{N}}\left(\hat{s}_{\text {TSLS }}-6\right)$ has the same limiting distribution as

$$
\sqrt{\mathbb{N}}\left[\alpha\left(\hat{\delta}_{1}-\delta ;+(1-\alpha)\left(\hat{\delta}_{2}-\delta\right)\right]\right.
$$

This suggests finding the $\tau$ that minimizes the variance of the limiting distribution of $\sqrt{\mathbb{N}}\left[\tau\left(\hat{\delta}_{1}-\delta\right)+(1-\tau)\left(\hat{\delta}_{2}-\delta\right)\right]$. The answer leads to the minimum 
distance estimator: choose $\hat{\theta}$ to

$$
\begin{aligned}
& \min _{\theta}\left[\left(\begin{array}{c}
\hat{\Sigma}_{1} \\
\hat{\delta}_{2}
\end{array}\right)-\left(\begin{array}{l}
\theta \\
\theta
\end{array}\right)\right] \stackrel{\Lambda}{ }^{-1}\left[\left(\begin{array}{c}
\hat{\delta}_{1} \\
\hat{\sigma}_{2}
\end{array}\right)=\left(\begin{array}{l}
\theta \\
\theta
\end{array}\right)\right] \\
& \hat{\theta}=\tau \hat{\delta}_{1}+(1-\tau) \hat{\delta}_{2},
\end{aligned}
$$

where

$$
\tau=\left(\lambda^{11}+\lambda^{12}\right) /\left(\lambda^{11}+2 \lambda^{12}+\lambda^{22}\right)
$$

and $\lambda^{j k}$ is the $j, k$ element of $A-1$. The estimator obtained by using a consistent estimator of $\Lambda$ has the same limiting distribution.

In general $\tau \neq$ a since $\tau$ is a function of fourth moments and $\alpha$ is not. Suppose, for example, that $\mathbf{z}_{\mathbf{i}}=\mathbf{x}_{\mathbf{i} 2}$. Then $\boldsymbol{\alpha}=0$ but $\boldsymbol{\tau} \neq 0$ unless

$$
E\left[\left(y_{i}-\delta z_{1}\right)^{2}\left(\frac{x_{12}^{2}}{E\left(x_{12}^{2}\right)}-\frac{x_{11} x_{12}}{E\left(x_{11} x_{12}\right)}\right)\right]=0 \text {. }
$$

If we add another equation, then we can consider the conventional

three-stage least squares estimator. Its limiting distribution is derived in the Appendix (Proposition 7); however, viewed as a minimum distance estimator, it is using the wrong norm in general.

Considar the standard simultaneous equations model:

$$
\begin{aligned}
& \underline{y}_{i}=\Pi \underline{x}_{-1}+\underline{u}_{i}, \quad E\left(\underline{u}_{i} x_{i}^{\prime}\right)=0, \\
& \Gamma_{\sim i}+B \underline{x}_{i}=\underline{v}_{1},
\end{aligned}
$$

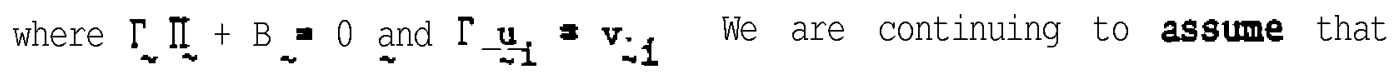

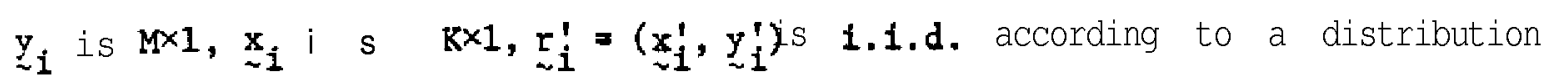
with finite fourth moments $(i=1, \ldots . . . N)$, and that $\mathbf{E}\left(\mathbf{x}_{\mathbf{i}} \mathbf{x}_{i}^{\prime}\right)$ is nonsingular. 
There arc restrictions on $\Gamma_{*}$ and $B: \underset{*}{\mathbb{m}}(\Gamma, \underset{\sim}{B})=0$, where $\mathbb{m}$ is a known function. Assume that the implied restrictions on II can be specified by the condition that $\pi=\operatorname{vec}\left(\Pi^{+}\right)=\underset{\sim}{f}(6)$, where the domain of $\delta$ is $T_{1^{\prime}}$, a subset of $\mathbb{R}^{s}$ that includes the true value $\delta^{\circ}(\mathbf{s} \leq \mathbf{M K})$. Assume that $\mathrm{Tl}$ and $\mathrm{f}$ satisfy assumptions 1 and 2 ; these properties could be derived from regularity conditions on $m$, as in Malinvaud (1970, proposition 2, p. 670).

$$
\text { Choose } \hat{\delta} \text { to }
$$

$$
\min _{\delta \varepsilon T_{1}}[\hat{\pi}-\underline{f}(\delta)] \hat{\Omega}^{-1}[\hat{\pi}-\underset{\sim}{\mathbf{f}}(\delta)],
$$

where $\hat{\Omega}$ is given in $(4.2)$ and we assume that $\Omega$ in $(4.1)$ is positive definite. Let $F=\partial \underset{\sim}{f}\left(\underline{\sim}^{0}\right) / \partial \delta^{\prime}$. Then we have $\left.\sqrt{\mathbb{N}}\left(\underset{\sim}{\hat{\delta}}-\underline{\sim}^{0}\right) \stackrel{D}{\longrightarrow} \mathbb{N}^{\prime} \underset{\sim}{0}, \underline{\sim}\right)$, where $\Lambda=\left(F^{\prime} \Omega^{-1} F\right)^{-1}$. This generalizes Malinvaud's minimum distanc: estimator (p. 676); it reduces to his estimator if $u_{1}^{0} \mathrm{o}^{\prime}$ is uncorrel: ad

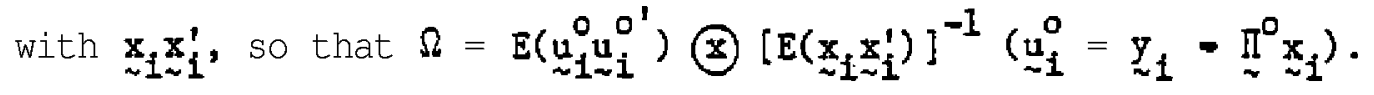
Now suppose that the only restrictions on $\Gamma$ and $\mathrm{B}$ are that certain coefficients are zero, together with the normalization restrictions that the coefficient of $\mathbf{y}_{\mathbf{i m}}$ in the $\frac{\mathbf{m} \text { th }}{-}$ structural equation is one. Then we can give $a^{*}$ explicit formula for $A$. Write the $\mathbf{m}$ th structural equation as

$$
y_{\text {im }}=\delta_{-m}^{\prime} z_{i m}+v_{i m},
$$

where the components of $\underset{\sim}{\mathbf{z} m}$ are the variables in $\underset{\sim}{\mathbf{1}}$ and ${\underset{\sim}{\mathbf{i}}}_{\mathbf{i}}$ that appear in the $\mathbf{m}$ equation with unknown coefficients. Let there be $\mathbb{M}$ structural equations and asṣme that the true value $\Gamma^{\circ}$ is nonsingular. Let $\delta^{\prime}=\left(\delta_{1}^{i}, \ldots, \delta_{\mathcal{M}}^{\prime}\right)$ be $\mathbf{s} \times \mathbf{I}$, and let $\underset{\sim}{\Gamma}(\delta)$ and $\underset{\sim}{\mathbf{B}}(\delta)$ be parametric representations of $\Gamma$ and $B$ that satisfy the zero restrictions and the normalization rule. We can choose a compact set $T_{1} \boldsymbol{C}_{\mathbf{R}}^{\mathbf{s}}$ containing a neighborhood 0 : the true value $\underline{\delta}^{\circ}$, such that $\underset{\sim}{\Gamma}(\delta)$ is nonsingular for $\delta \varepsilon \mathrm{T}_{\perp}$. Then $\pi=\underset{\sim}{f}(\delta)$, 
where $\underset{\sim}{\mathrm{f}}(\hat{0})=\operatorname{vec}\left[-\Gamma^{-1}(\hat{0}) \mathrm{B}(\hat{0})\right]^{\prime}$.

Assume that $\underset{\sim}{f}(\underset{\sim}{6})=\prod^{0}$ implies that $\underset{\sim}{0}=\underbrace{0}_{\sim}$, so that the structural parameters are identified. Then $\mathrm{T}_{1}$ and $\mathrm{f}$ satisfy Assumptions 1 and 2 , and

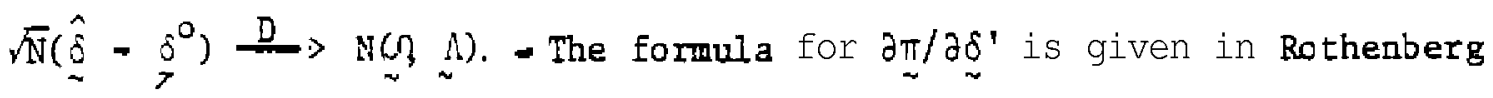
(1973, p. 69):

$$
\frac{\partial \pi}{\partial \delta},=-\left(\Gamma^{-1}(\otimes) \underset{\sim R}{I_{R}}\right)\left[\underset{\sim 2 x}{\Phi}\left(I_{M}(x) \Phi_{-x}^{-1}\right)\right]^{\prime},
$$

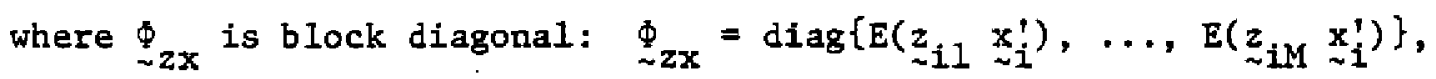
and $\Phi_{x}=E\left(x_{1} x_{i}^{\prime}\right)$. So we have

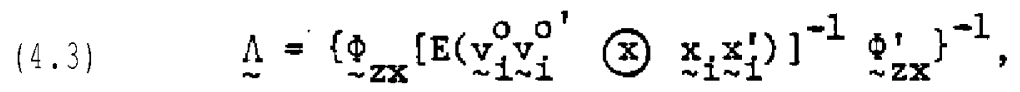

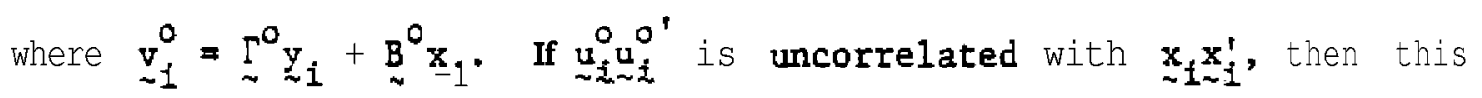
reduces to

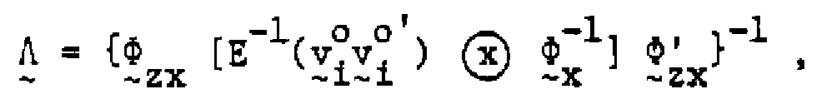

which is the conventional asymptotic covariance matrix for three-stage least squares (Zellner and ThieI (1962)).

I shall present a generalization of three-stage least squares that has the same limiting distribution as the generalized minimum distance estimator. Let $\beta=\operatorname{vec}\left(B^{\prime}\right)$ and note that $\pi=-\left(\Gamma^{-1}(\boldsymbol{x} \underset{\sim}{\Omega}) \underline{\sim}\right.$. Then we have

$$
\begin{aligned}
& {\left[\hat{\pi}+\left(\underline{\sim}^{-1} \otimes \underset{\sim}{I}\right) \underset{\sim}{\beta}\right]^{\prime} \underline{\Omega}^{-1}\left[\underset{\sim}{\pi}+\left({\underset{\sim}{\Gamma}}^{-1} \otimes \underset{\sim}{\beta} \underset{\sim}{\beta}\right]\right.}
\end{aligned}
$$

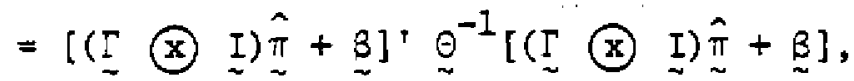


where

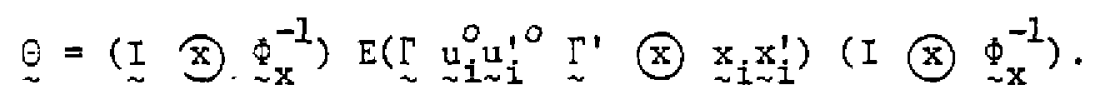

Let $\mathbf{s}_{\mathbf{z x}}$ be the following block-diagonal matrix:

$$
S_{\sim z x}=\operatorname{diag}\left\{\frac{1}{N} \sum_{i=1}^{N} z_{i=1}^{N} x_{i}^{\prime}, \ldots \cdot \frac{1}{N} \sum_{i=1}^{N} z_{\sim 1 M} x_{i}^{\prime}\right\}
$$

and let

$$
s_{\sim x}=\frac{1}{N} \sum_{i=1}^{N} x_{i \sim 1}^{N} x_{i}^{\prime}, \quad s_{\sim x y}=\frac{1}{N} \sum_{i=1}^{N} \underline{y}_{i} \otimes x_{i}
$$

Let

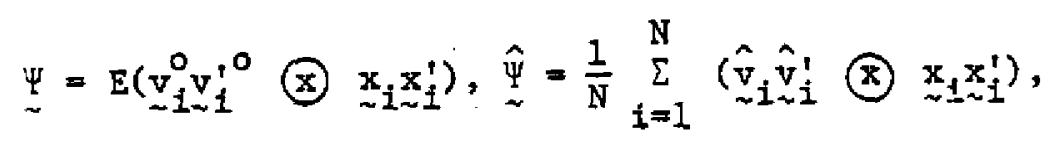

where

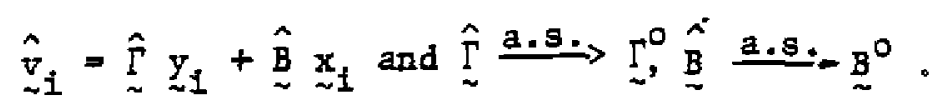

Now replace $\theta$ by

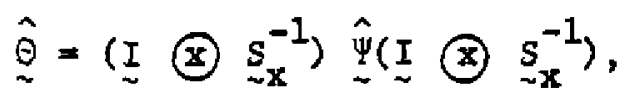

and note that

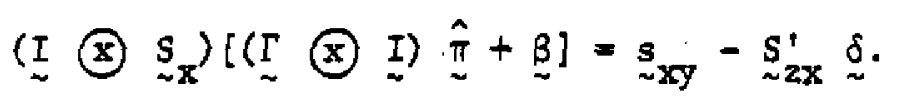

Then we have the following distance function:

$$
\left(s_{x y}-s_{\sim 2 x}^{\prime} \delta\right)^{\prime} \underline{\sim}^{-1}\left(s_{x y}-s_{\sim z x}^{\prime} \delta\right) .
$$

This corresponds to Basmann's (1965) interpretation of three-stage least squares. 17 


$$
\begin{aligned}
& \text { Minimizing with respect to } 2 \text { gives }
\end{aligned}
$$

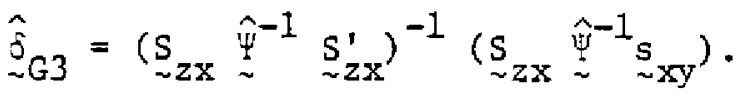

The limiting distribution of this estimator is derived in the Appendix (Proposition 7). We record it es

Proposition 4. $\quad \sqrt{\mathbb{N}}\left(\stackrel{\delta}{\sigma}_{G} 3-\delta^{\circ}\right) \stackrel{D}{\longrightarrow} \mathbb{N}(\underset{\sim}{0}, \underset{\sim}{N})$, where $A$ is given in (4.3). This generalized three-stage least squares estimator is asymototically efficient within the class of minimum distance estimators.

Our derivation of the limiting distribution of $\hat{\delta}_{-\mathrm{G} 3}$ relies on linearity. For a generalized nonlinear three-stage least squares estimator, see Hansen (1982).

Finally, we shall consider the generalization of two-stage least squares. 38 Suppose that

$$
y_{11}=\delta_{i}^{i} z_{i 1}+v_{11}
$$

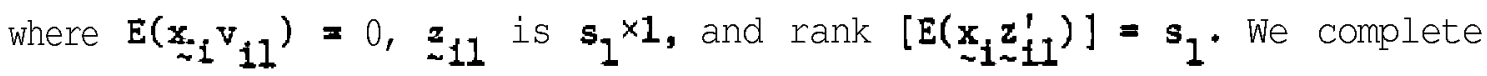
the system by setting

$$
y_{i m}=\pi_{i m}^{*} x_{i}+u_{1 m}
$$

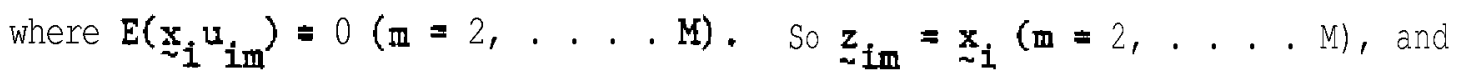

$$
\Phi_{2 X}=\operatorname{dlag}\left\{E\left(z_{i 1} \underset{\sim i}{x_{i}^{i}}\right), \quad I_{M-1}(x) E\left(\underline{x}_{1} x_{i}^{\prime}\right)\right\}
$$

Let $5^{\prime}=\left(\delta_{1}^{\prime}, \pi_{2}^{\prime}, \ldots, \pi_{M}^{\prime}\right)$ and apply the minimum distance procedure to obtain $\hat{\tilde{J}}$; since we are ignoring any restrictions on $\underset{\sim \mathbb{m}}{\vec{\pi}}(\mathbf{m}=\mathbf{2}, \ldots ., \mathbf{M})$, 
$\hat{\hat{c}}$ is a limited information minimum distance estimator.

We have $\sqrt{\mathbb{N}}\left(\hat{\sigma}_{-1}^{\hat{\delta}_{1}}-{\underset{\sim}{\delta}}_{1}^{\circ}\right) \stackrel{D}{\longrightarrow} \mathbb{N}\left(\underset{\sim}{0}, \Lambda_{11}\right)$, and evaluating the partitioned inverse gives

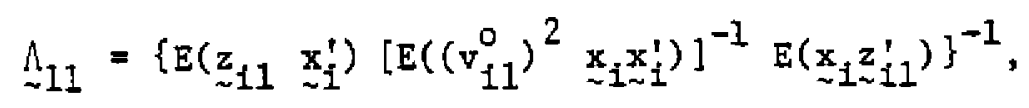

where $v_{11}^{0}=y_{i 1}-0_{i 1}^{0}{ }_{i}^{0}$.

We can obtain the same limiting distribution by using the following generalization of two-stage least squares: Let

$$
\begin{aligned}
& z_{1}^{\prime}=\left(z_{i 1}, \ldots, z_{N 1}\right), x^{\prime}=\left(x_{1}, \ldots, x_{N}\right), \\
& y_{i}^{\prime}=\left(y_{11}, \ldots, y_{N 1}\right), \text { and } \\
& \hat{\Psi}_{-11}=\frac{1}{N} \sum_{i=1}^{N}\left(y_{i 1}-\hat{\delta}_{i}^{\prime} z_{i 1}\right)^{2} x_{i \sim 1} x_{i}^{\prime},
\end{aligned}
$$

where $\hat{\delta}_{\mathbf{l}} \stackrel{\text { a.s.s. }}{\rightarrow} \delta_{-1}^{0}$ (for example, $\hat{\delta}_{\mathbf{l}}$ could be an instrumental variable estimator); then

$$
\hat{\delta}_{I G 2}=\left(z_{i}^{\prime} x \hat{\Psi}_{-11}^{-1} \underset{\sim}{x^{\prime} z_{1}}\right)^{-1}\left(z_{1}^{\prime} \underset{\sim}{x} \hat{\Psi}_{-11}^{-1} x^{\prime} \underline{\sim}_{1}\right)
$$

This is the estimator of $\delta_{1}$ that we obtain by applying generalized threestage least squares to the completed system, with no restrictions on $\prod_{-\mathbb{m}}(\mathbf{m}=2, \ldots . \mathrm{M})$. The limiting distribution of this estimator is derived in the Appendix (Proposition 7): 


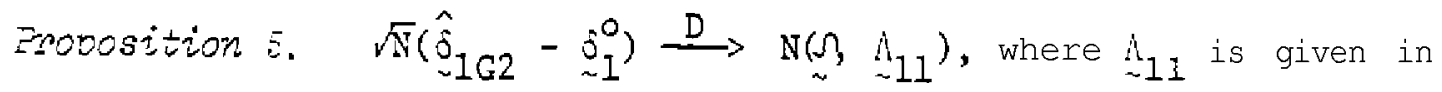
$(4.4)$. This generalized two-stage least squares estimator is asyoptotically efficient in the class of limited information minimum distance estimators.

\subsection{Asymptotic Efficiency: A Comparison with the Quasi-Aarimum Likelihood Estinator}

Assume that $\mathbf{r}_{\boldsymbol{i}}$ is $\mathbf{1} . \mathbf{1} . \mathbf{d} . \quad(\mathbb{1}=\mathbf{1}, \mathbf{2}, \ldots)$ from a distribution with $\mathbf{E}\left(\underline{\tau}_{\mathbf{i}}\right)=\tau, \mathrm{V}\left(\underline{\boldsymbol{r}}_{\mathbf{1}}\right)=\Sigma$, where $\Sigma$ is a $\mathrm{J} \times \mathrm{J}$ positive-definite matrix; the fourth moments are finite. Suppose that we wish to estimate functions of $\Sigma$ subject to restrictions. Let $\sigma=\operatorname{vec}(\boldsymbol{\Sigma})$ and express the restrictions by the condition that $\sigma=\underset{g}{\mathbf{g}}(\theta)$, where $\boldsymbol{g}$ is a function fr $T=$ ito $\mathbf{R}^{\mathrm{q}}$ with $\mathrm{a}$ domain $T \subset \mathbb{R}^{p}$ that contains the true value $\left.\theta^{\circ}\left(q=J^{2} ; j \leq J j+1\right) / 2\right)$. Let

$$
\bar{s}=\frac{1}{N} \sum_{i=1}^{N}\left(\underline{r}_{i}-\bar{r}\right)\left(\underline{r}_{i}-\bar{r}\right)^{v},
$$

and let $\overline{\mathbf{s}}=\operatorname{vec}(\overline{\mathbf{s}})$.

If the distribution of $\mathfrak{r}_{\mathfrak{1}}$ is multivariate normal, then the log-likelihood function is

$$
L=\frac{N}{2} \ln \left|\underline{\Sigma}^{-1}\right|-\frac{N}{2} \operatorname{tr}\left\{\Sigma^{-1}\left[\tilde{S}+(\overline{\underline{L}}-\tau)(\overline{\underline{\tau}}-\tau)^{\prime}\right]\right\} .
$$

If there are no restrictions on $\tau$, then the maximum likelihood estimator of $\theta^{\circ}$ is a solution to the following problem: Choose $\hat{\theta}$ to soive

$$
\frac{\partial \underline{g}^{\prime}(\underline{\theta})}{\partial \theta}\left[\Sigma^{-1}(\underline{\theta}) \Theta \underline{\Sigma}^{-1}(\underset{\sim}{\theta})\right](\underline{\mathbf{s}}-g(\underline{\theta}))=0_{\sim} .
$$

We shall derive the properties of this estimator when the distribution of 
$r_{i}$ is not necessarily normal; in that case we shall refer to the estimator

as a quasi-maximum likelihood estimator $\left(\hat{\vartheta}_{\text {QML }}\right){ }^{39}$

Macurdy (1979) considered a version of this problem and showed that, under suitable regularity conditions, $\sqrt{\mathbb{N}\left(\hat{\theta}_{\text {QML }}\right.}-\underbrace{\circ}_{\sim})$ has a limiting normal distribution; the covariance matrix, however, is not given by the standard information matrix formula. We would like to compare this distribution with the distribution of the minimum distance estimator.

This 'comparison can be readily made by using theorem 1 in Ferguson (1958). In our notation, Ferguson considers the following problem: Choose $\hat{\theta}$ to solve

$$
W(\bar{s}, \theta)[\bar{s}-g(\theta)]=0
$$

He derives the limiting distribution of $\sqrt{\mathrm{N}}\left(\hat{\theta}-{\underset{\sim}{*}}^{\circ}\right)$ under regularity conditions on the functions $w_{\sim}$ and $g_{v}$. These regula ity conditions are particularly simple in our problem since $w$ does not depend on $\vec{s}$. We can state them as follows:

Assumption 3. $\Xi_{0} \subset \mathbf{R}^{\mathrm{p}}$ is an open set containing $\underline{\theta}^{\circ} ; \mathrm{g}$ is a continuous, one-to-one mapping of $\Xi_{0}$ into $\mathbf{R}^{\mathbf{q}}$ with a continuous inverse; $g$ has continuous second partial derivatives in $\Xi_{0}$; $\operatorname{rank}\left[\partial \underset{\sim}{g}(\theta) / \partial \theta_{\sim}^{\prime}\right]=p$ for $\underset{\sim}{\theta \varepsilon} \Xi_{0} ; \underset{\sim}{\Sigma}(\theta)$ is nonsingular for $\theta \varepsilon \Xi_{0}$.

In addition, we shall need $\overline{\mathbf{s}} \stackrel{\mathbf{a . s}}{\longrightarrow} \mathbf{g}\left(\theta^{\circ}\right)$ and the central 1imit theorem result

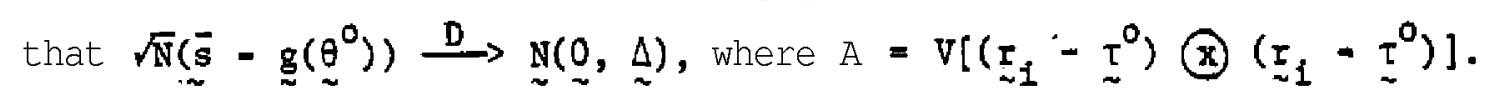

Then Ferguson's theorem implies that the likelihood equations almost surely have a unique solution within $\Xi_{0}$ for sufficiently large $\mathbf{N}$, and $\sqrt{\mathbb{N}}\left(\hat{\theta}_{\sim}{ }_{Q L}-e^{\circ}\right) \stackrel{D}{\longrightarrow} \mathbb{N}(0, \Lambda)$, where 


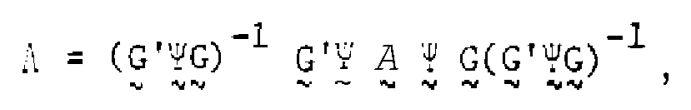

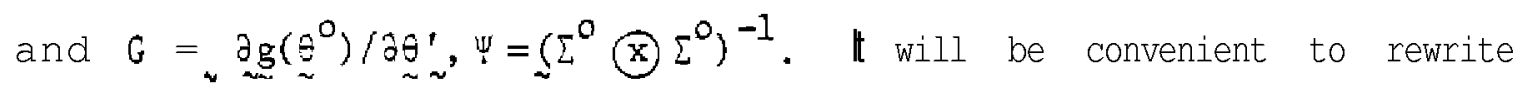
this, imposing the symmetry restrictions on $\Sigma$. Let $\sigma^{*}$ be the $J(J+1) / 2 \times 1$ vector formed by stacking the columns of the lower triangle of $\Sigma$. We can define a $\mathrm{J}^{2} \times[\mathrm{J}(\mathrm{J}+1) / 2]$ matrix $\mathrm{T}$ such that $\sigma=\mathrm{T} \sigma_{*}^{*}$. The elements in each row of $T$ are all zero except for a single element which is one; $T$ has full

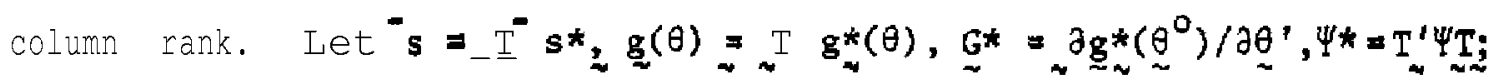
then $\sqrt{\mathbf{N}}\left[\overline{\mathbf{s}}^{*}-\mathbf{g}^{*}\left(\theta^{0}\right)\right] \stackrel{\mathrm{D}}{\longrightarrow} \mathbf{N}\left(\underline{0}, A^{*}\right)$, where $A^{*}$ is the covariance matrix of the vector formed from the columns of the lower triangle of $\left(\boldsymbol{r}_{\boldsymbol{i}}-\tau^{0}\right)\left(\boldsymbol{\tau}_{\mathbf{i}}=\tau^{0}\right)^{\prime}$. Now we can set

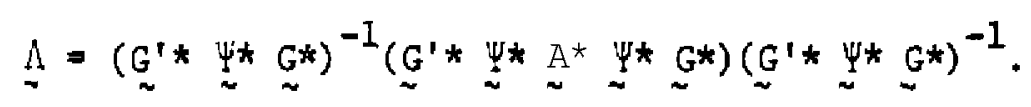

Consider the following minimum distance estimator: Choose $\hat{\theta}_{M D}$ to

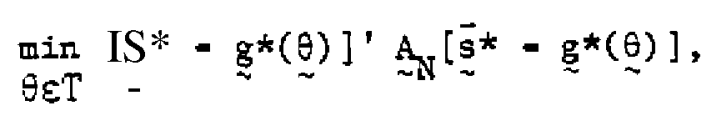

where $T$ is a compact subset of $\Xi_{0}$ that contains a neighborhood of $\theta^{0}$ and $\underset{\boldsymbol{N}}{\mathbf{a} . \mathbf{s}} \stackrel{\boldsymbol{\Psi}}{\boldsymbol{*} *}$. Then the following result is implied by Proposition 2 . Proposition 6. If Assumption 3 is satisfied, then $\sqrt{\mathbb{N}}\left(\hat{\theta}_{\alpha \mathbf{Q L}}-\stackrel{\theta}{\sim}^{\circ}\right)$ has the same limiting distribution as $\sqrt{\mathbb{N}}\left(\hat{\theta}_{\mathbb{M D}}-\underline{\theta}^{\circ}\right)$.

If $A^{*}$ is nonsingular, an optimal minimum distance estimator has $\underset{\sim \mathbb{N}}{\stackrel{\text { a.s. }}{\longrightarrow} \boldsymbol{\zeta} \Delta_{\boldsymbol{*}}^{*}}{ }^{-1}$, where $\zeta$ is an arbitrary positive real number. If the distribution of $\underset{\sim}{\mathbf{i}}$ is normal, then $A^{\star^{-1}} \mathbf{x}(1 / 2) \Psi^{*}$; but in general $\Delta^{*}$ is not proportional to $\underset{\Psi *}{*}$, since $A^{*}$ depends on fourth moments and $\Psi^{*}$ is a function of second moments. 
So in general $\hat{G}_{\sim \mathrm{MLL}}$ is less efficient than the optimal minimum distance estimator that uses

$$
\Delta_{N}=\left[\frac{I}{N} \sum_{i=1}^{N}\left(s_{i}^{*}-\bar{s}^{*}\right)\left(s_{i}^{*}-\bar{s}^{*}\right)^{\prime}\right]^{-1},
$$

where $\underset{\sim \mathbf{i}}{\mathbf{i}}$ is the vector formed from the lower triangle of $\left({\underset{\sim}{\mathbf{i}}}_{\mathbf{i}}-\overline{\mathbf{r}}\right)(\underset{\sim}{\mathbf{i}}-\overline{\mathbf{r}})$ '.

More generally, we can consider the class of consistent estimators that are continuously differentiable functions of $\overline{\mathbf{s}}_{\sim}^{*}: \hat{\theta}_{\sim}=\hat{\theta}\left(\overline{\mathbf{s}}^{*}\right)$. Chiang (1956) shows that the minimum distance estimator based on $\Delta^{*}$-1 has the minimal asymptotic covariance matrix within this class. The minimum distance estimator based on $\mathrm{A}_{\mathbf{N}}$ in (4.5) attains this lower bound.

\subsection{Multivariate Probit Models}

Suppose that

$$
\begin{aligned}
y_{i m} & =1 \text { if } \prod_{-m}^{\prime} x_{i}+u_{i m} \geq 0, \\
& =0 \text { otherwise }(i=1, \ldots, N \text {; m=l, . . . . M), }
\end{aligned}
$$

where the distribution of $u_{-\mathbf{I}}=\left(\mathbf{u}_{\mathbf{I}}, \ldots, \mathbf{u}_{\mathbf{i} \mathbf{M}}\right)$ conditional on $\mathbf{x}_{\mathbf{i}}$ is multivariate normal, $\mathbf{N}(\mathbf{O}, \Sigma)$. There may be restrictions on $\pi^{\prime}=\left(\pi_{-1}^{\prime}, . . . . \pi_{\mathbf{M}}^{\prime}\right)$, but we want to allow $\underset{\sim}{\Sigma}$ to be unrestricted, except for the scale normalization that the diagonal elements of $\Sigma$ are equal to one. In that case, the maximum likelihood estimator has the computational disadvantage of requiring numerical integration over $\mathrm{M}-1$ dimensions.

our strategy is to avoid numerical integration. We estimate $\underset{\sim \mathbf{m}}{\pi}$ by maximizing the marginal likelihood function that is based on the distribution of $y_{\text {im }}$ conditional on $x_{-1}$ ' 


$$
P\left(y_{i m}=1 \mid x_{i 1}\right)=F\left(\underset{-m}{\pi^{\prime}} x_{i}\right)
$$

where $\mathrm{F}$ is the standard normal distribution function. Then-under standard

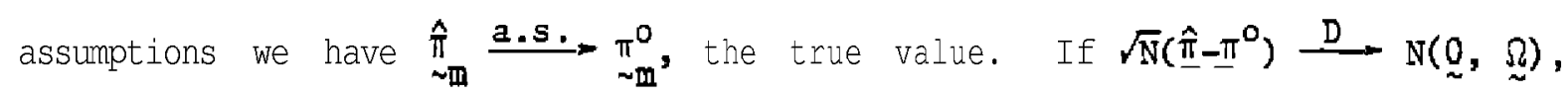
then we can impose the restriction that $\pi \mathbf{m} \underset{\sim}{f(\delta)}$ by choosing $\underset{\sim}{\hat{\delta}}$ to minimize

$$
[\hat{\pi}-\underset{\sim}{f}(\delta)]^{\prime} \hat{\Omega}^{-1}[\underset{\sim}{\tilde{\pi}}-\underset{\sim}{f}(\underset{\sim}{\delta})] .
$$

We only need to derive a formula for $\Omega .40$

our estimator of $\underset{\sim}{\pi}$ is solving the following equation:

$$
\mathbf{s}(\hat{\pi})=\frac{\partial Q(\hat{\pi})}{\partial \pi}=\underset{\sim}{0}
$$

where

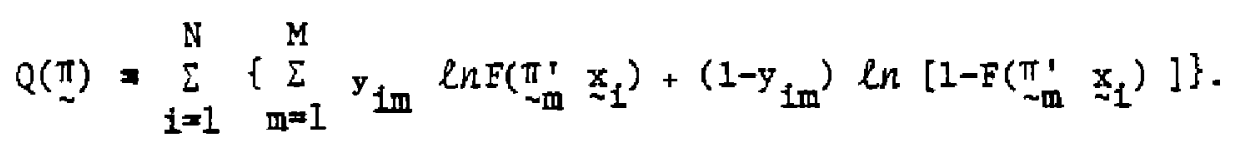

Hence the asymptotic distribution of $\hat{\pi}$ can be obtained from the theory of "M-estimators." Huber (1967) provides general results, which do not impose differentiability restrictions on $\mathbf{s}(\boldsymbol{\pi})$. His results cover. for example, regression estimators based on minimizing the residual sum of absolute deviations. We shall not need this generality here and shall sketch the derivation for the simpler, differentiable case. This case has been considered by Hansen (1982), Macurdy (1981a), and white (1982). ${ }^{4}$

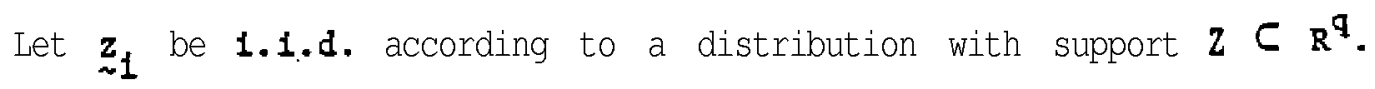
Let $\theta$ be an open, convex subset of $\mathbf{R}^{\mathbf{P}}$ and let $\underset{\sim}{\boldsymbol{\Psi}}(\mathbf{z}, \underset{\sim}{\boldsymbol{\theta}})$, be a function from $z \times \theta$ into $\mathbf{R}^{\mathrm{p}}$; its $\mathbf{k} \frac{\text { th }}{\mathbf{z}}$ component is $\psi_{\mathbf{k}}(\mathbf{z}, \theta)$. For each $\underset{\sim}{\theta} \boldsymbol{\theta}, \underset{\sim}{\Psi}$ is a measurable function of $\mathbf{z}$, and there is a $\theta^{\circ} \varepsilon$ with 


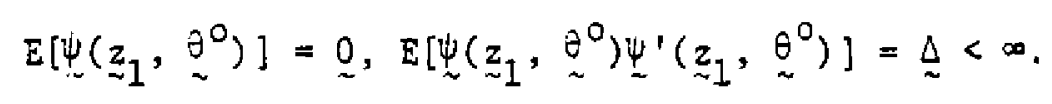

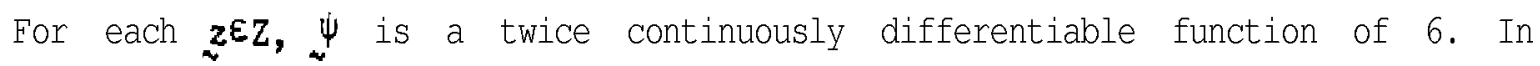
addition,

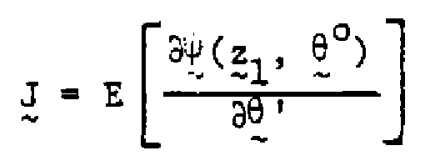

is nonsingular, and

$$
\left|\frac{\partial \psi_{k}^{2}\left(z,{ }_{z}\right)}{\partial \theta} \ell^{\partial \theta}\right| \leq h(z) \quad(k, \ell, m=1, \ldots, p)
$$

for $\underset{\sim}{\theta} \varepsilon \theta$, where $\mathrm{E}\left[\mathrm{h}\left(\mathrm{z}_{1}\right)\right]<\infty$.

Suppose that $r \geq$ have a (measurable) estimator $\underset{\sim \mathbb{N}}{\hat{\theta}} \in \theta$ such that $\underset{\sim \mathbb{N}}{\hat{\theta}} \stackrel{\text { a.s.s. }}{\longrightarrow} \stackrel{\theta}{\sim}^{0}$ and

$$
\sum_{i=1}^{N} \Psi\left(z_{\sim i}, \hat{\theta}_{\sim N}\right)=2
$$

for sufficiently large $\mathbf{N}$ a.s. By Taylor's theorem,

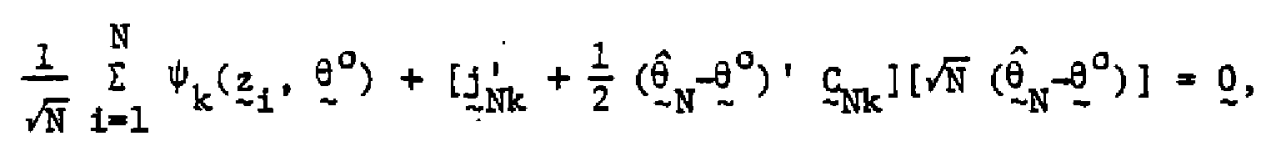

where

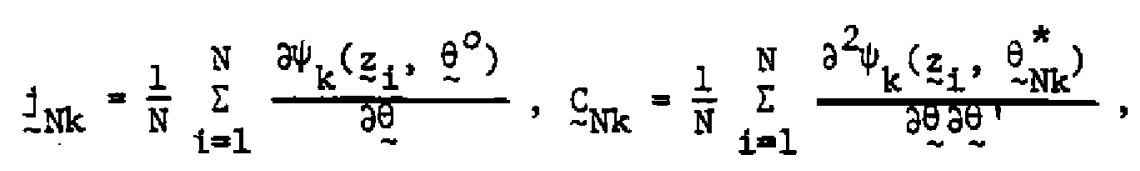

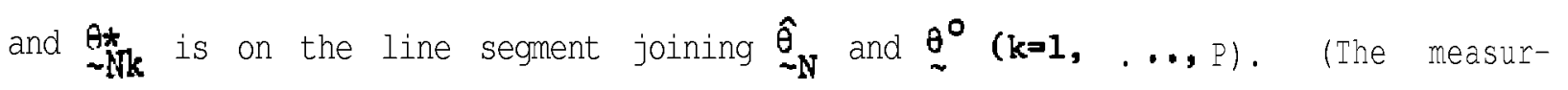
ability of $\underset{\sim \mathbb{N}}{\theta *}$ follows from lemna 3 of Jennrich (1969).) By the strong law

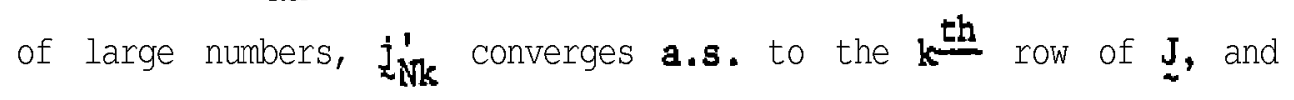




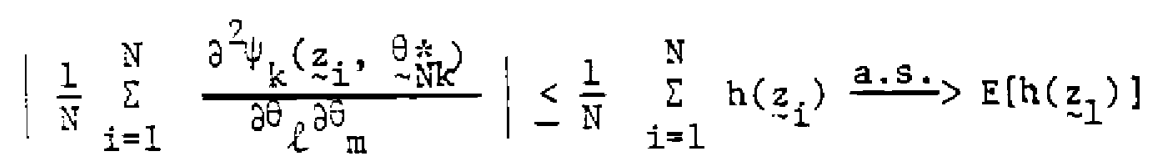

$(\mathrm{k}, \ell, \mathrm{m}=1, \ldots . . \mathrm{p})$. Hence $\left(\hat{\theta}_{\sim \mathrm{N}}-\theta^{\circ}\right)^{\prime} \underset{\sim \mathrm{Nk}}{\mathrm{C}_{\mathrm{Nk}}}+\underset{\sim}{\mathrm{a} . \mathrm{s}}$. and

$$
\sqrt{\mathrm{N}}\left(\hat{\theta}_{\sim \mathrm{N}}-\underline{\theta}^{0}\right)=-\mathrm{D}_{\sim \mathrm{N}}^{-1}\left[\frac{1}{\sqrt{\mathrm{N}}} \sum_{i 1=1}^{\mathrm{N}} \underset{\sim}{\sim}\left(z_{i}, \stackrel{\theta}{\sim}^{0}\right)\right]
$$

for $N$ sufficiently large a.s., where $D_{N} \stackrel{\text { a.s. }}{\longrightarrow}$ J. By the central limit theorem,

$$
\frac{1}{\sqrt{N}} \sum_{i=1}^{N} \Psi\left(z_{i}, \theta^{\circ}\right) \stackrel{D}{\longrightarrow} N(\underline{0}, \Delta)
$$

Hence

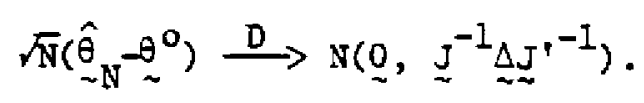

Applying this result to our multivariate probit estimator gives

$$
\sqrt{\mathrm{N}}\left(\underset{\sim}{\pi}-\underline{\pi}^{0}\right) \stackrel{\mathrm{D}}{\longrightarrow} \mathrm{N}\left(\underline{0},{\underset{\sim}{J}}^{-1} \underline{\Delta \sim}_{\sim}^{-1}\right)
$$

where $\mathbf{J}=\operatorname{diag}\left[J_{-1}, \ldots, I_{\mathbf{M}}\right\}$ is a block-diagonal matrix with

$$
J_{-\mathrm{II}}=E\left[\left\{\left(F^{\prime}\right)^{2} /[F(1-F)]\right\} \quad x_{1} x_{1}^{i}\right]
$$

(F and its derivative $F^{\prime}$ are evaluated at $\pi_{\sim \pi}^{\circ} x_{1}^{\prime}$ );

and

$$
\left.\triangleq=E[\underset{\sim}{H} \mathbb{x}) \underset{\sim}{x_{1}^{\prime}}\right]
$$

where the $\mathbf{m}, \mathbf{n}$ element of the $\mathbf{M} \times \mathbf{M}$ matrix $\underset{\sim}{\mathrm{H}}$ is $\mathbf{h}_{\mathbf{m}} \mathbf{z} \mathbf{e}_{\mathbf{m}} \mathbf{e}_{\mathbf{n}}$ with

$$
\mathrm{e}_{\mathrm{m}}=\frac{\mathrm{y}_{1 \mathrm{~m}}-\mathrm{F}}{\mathrm{F}(1-\mathrm{F})} \mathrm{F}^{\prime} \quad(\mathrm{II}=1 \ldots, \mathrm{M})
$$


( $F$ and $F^{\prime}$ are evaluated at $\underset{\sim \mathbf{m}}{\pi^{\circ} \mathbf{x}_{1}}$ ). We obtain a 'consistent estimator $(\underset{\sim}{\hat{\Omega}})$ of $\mathfrak{J}^{-1} \Delta J^{-1}$ by replacing expectations by sample means and using $\underset{\sim}{\hat{\pi}}$ in place of $\pi^{0}$. Then we can apply the minimum distance theory of section 4.2 to impose restrictions on $\pi$.

\section{EMPIRICAL APPLICATIONS}

\subsection{Linear Models: Union Wage Effects}

We shall present an empirical example that illustrates some of the preceding results. ${ }^{42}$ The data come from the panel of Young Men in the National Longitudinal Survey (Parnes). The sample consists of 1454 young men who were not enrolled in school in 1969, 1970, or 1971, and who had complete data on the variables listed in Table 1. Table 2.1 presents an unrestricted least squares regression of the logarithm of wage in 1969 on the union, SMSA, and region variables for all three years. The regression also includes a constant, schooling, experience, experience squared, and race. This regression is repeated using the 1970 wage and the 1971 wage.

In Section 2 we discussed the implications of a random intercept (c). If the leads and lags are due just to $c$, then the submatrices of $I$ corresponding to the union, SMSA, or region coefficients should have the form $\beta I+\ell \lambda^{\prime}$. Consider, for example, the $3 \times 3$ submatrix of union coefficients -the off-diagonal elements in each column should be equal to each other. So we compare .048 to $.046, .042$ to .041 , and'-.009 to .010 ; not bad.

In Table 2.2 we add a complete set of union interactions, so that, for' the union variables at ieast, we have a general regression function. Now the submatrix of union coefficients is $3 \times 7$. If it equals $\left(8 \mathrm{I}_{3}, 0\right)+\ell \lambda^{\prime}$, then 
Table 1

CEAPACTERISTICS OF NATTONAL LONGTMUDINAL SURVEY YOUNG
VEN, NOT ENROLLED IN SCHOOL IN 1969, 1970, 1971:

Means and Standard Deviations

$$
N=1454
$$

Variable

Mean Standard Deviation

\begin{tabular}{lrr} 
LW1 & 5.64 & .423 \\
LW2 & 5.74 & .426 \\
LW3 & 5.82 & .437 \\
U1 & .336 & \\
u2 & .362 & \\
u3 & .364 & \\
U1U2 & .270 & \\
U1U3 & .262 & \\
U2U3 & .303 & \\
U1U2U3 & .243 & \\
SMSA1 & .697 & \\
SMSA2 & .627 & \\
SMSA3 & .622 & \\
RNS1 & .409 & 2.64 \\
RNS2 & .404 & 3.71 \\
RNS3 & .410 & 46.6 \\
S & 11.7 & \\
EXP69 & 5.11 & \\
EXP692 & 39.8 & \\
RACE & .264 & \\
\hline
\end{tabular}

Notes to Table 1:

LW1, LW2, LW3 - logarithm of hourly earnings (in cents) on the current or last job in 1969, 1970, 1971; U1, U2, U3 -- I if wages on current or last job set by collective bargaining, 0 if not, in 1969, 1970, 1971; SMSA1, SMSA2, SMSA3 - 1 if respondent in SMSA. 0 if not, in 1969, 1970, 1971; RNS1, RNS2, RNS3 -- 1 if respondent in South, 0 if not, in 1969, 1970, 1971; s -- years of schooling completed; EXP69 -- (age in 1969 - S - 6); RACE -- 1 if respondent black, 0 if not. 
TABLE 2

UNRESTRICTED LEAST SQUARES REGEESSIONS

$\underline{2.1}$

Coeficients (and Standard Errors) of:

\begin{tabular}{lccc|ccccccc}
$\begin{array}{l}\text { Dependent } \\
\text { Variable }\end{array}$ & $\mathrm{U1}$ & $\mathrm{U} 2$ & $\mathrm{U} 3$ & SMSAI & SMSA2 & SMSA3 & RNS1 & RNS2 & RNS3 \\
\hline LW1 &. .171 & .042 & -.009 & .135 & -.001 & .032 & -.016 & -.020 & -.108 \\
& $(.025)$ & $(.026)$ & $(.025)$ & $(.028)$ & $(.055)$ & $(.054)$ & $(.081)$ & $(.081)$ & $(.070)$ \\
LW2 & .048 & .150 & .010 & .086 & .053 & .020 & .065 & -.039 & -.155 \\
& $(.023)$ & $(.028)$ & $(.026)$ & $(.027)$ & $(.065)$ & $(.061)$ & $(.099)$ & $(.109)$ & $(.092)$ \\
LW3 & $(.046$ & .041 & .132 & .083 & .003 & .088 & .074 & .056 & -.0232 \\
& $(.023)$ & $(.030)$ & $(.030)$ & $(.031)$ & $(.058)$ & $(.056)$ & $(.079)$ & $(.093)$ & $(.078)$ \\
\hline
\end{tabular}

Notes to Table 2.1:

All regressions include ( $\left.1,5, \operatorname{EXP} 69, \operatorname{EXP} 69^{2}, R A C E\right)$. The standard errors are calculated using $\Omega$ in $(4.2)$.

Coefficients (and Standard Errors) of:

\begin{tabular}{|c|c|c|c|c|c|c|c|}
\hline \multirow{2}{*}{$\begin{array}{l}\text { Dependent } \\
\text { Variable }\end{array}$} & \\
\hline & U1 & $\mathrm{U} 2$ & 43 & J1U2 & Ulu3 & $\mathrm{J} 2 \mathrm{U} 3$ & U1U2U3 \\
\hline LW1 & $\begin{array}{l}.127 \\
(.044)\end{array}$ & $\begin{array}{l}-.047 \\
(.042)\end{array}$ & $\begin{array}{l}-.072 \\
(.041)\end{array}$ & $\begin{array}{l}.128 \\
(.072)\end{array}$ & $\begin{array}{l}.092 \\
(.075)\end{array}$ & $\begin{array}{c}.156 \\
(.070)\end{array}$ & $\begin{array}{c}-.182 \\
(.104)\end{array}$ \\
\hline LW2 & $(.040)$ & $\begin{array}{c}.014 \\
(.045)\end{array}$ & $\begin{array}{l}-.085 \\
(.040)\end{array}$ & $\begin{array}{c}.181 \\
(.074)\end{array}$ & $\begin{array}{c}.118 \\
(.092)\end{array}$ & $(.0227)$ & $\begin{array}{l}-.229 \\
(.116)\end{array}$ \\
\hline LW3 & $\begin{array}{l}-.050 \\
(.037)\end{array}$ & $\begin{array}{l}-.072 \\
(.053)\end{array}$ & $\begin{array}{l}-.022 \\
(.052)\end{array}$ & $\begin{array}{l}.110 \\
(.079)\end{array}$ & $\begin{array}{c}.264 \\
(.081)\end{array}$ & $\begin{array}{l}.246 \\
(.079)\end{array}$ & $\begin{array}{l}-.256 \\
(.113)\end{array}$ \\
\hline
\end{tabular}

Notes to Table 2.2 :

All regressions include (SMSAl, SMSA2, SMSA3, RNS1, RNS2, RNS3, 1, S, EXP69, EXP69:, RACE). The standard errors are calculated using $\hat{\Omega}$ in $(4.2)$. 
in the first three columns, the off-diagonal elements within a column should be equal; in the last four columns, all elements within a column should be equal.

I first imposed the restrictions on the SMSA and region coefficients, using the minimum distance estimator. $\Omega$ is estimated using the formula in (4.2), and ${\underset{\sim}{\mathrm{N}}}_{\mathrm{N}}={\underset{\tilde{\Omega}}{ }}^{-1}$. The minimum distance statistic (Proposition 3) is 6.82, which is not a surprising value from a $x^{2}(10)$ distribution. If we impose the restrictions on the union coefficients as well, then the 21 coefficients in Table 2.2 are replaced by 8 : one $\beta$ and seven $\lambda$ 's. This gives an increase in the minimum distance statistic (Proposition $3^{\prime}$ ) of $19.36=6.82=12.54$, which is not a surprising value from a $\bar{x}^{2}(13)$ distribution. So there is no evidence here $\mathbf{a}_{\text {inst }}$ ins the hypothesis that all the lags and leads are generated by $\mathbf{c}$. In the erminology of Section 3.3, the (linear predictor) relationshi of $\mathbf{x}$ to $y$ arpears to be static conditional on $\boldsymbol{c}$. Consider a transformation of the model in which the dependent variables are LW1, LW2-LW1, and LW3-LW2. Start with a multivariate regression on all of the lags and leads (and union interactions); then impose the restriction that U, SMSA, and RNS appear in the LW2-LW1 and LW3-LW2 equations only as contemporaneous changes $\left(E\left(y_{t}-y_{t-1} \mid x_{1}, x_{2}, x_{3}\right)=\beta\left(x_{t}-x_{t-1}\right)\right)$. This is equivalent to the restriction that $\mathbf{c}$ generates all of the $\mathbf{l a g}:$ and leads, and we have seen that it is supported by the data. I also considered imposing all of the restrictions with the single exception of allowing separate coeficients for entering and leaving union coverage in the wage change equations. The estimates (standard errors) are .097 (.019) and 
$-.119(.022)$. The standard error on the sum of the coefficients is .024 , so again there is no evidence against the simple model with $E\left(y_{\mathbf{t}} \mid \mathbf{x}_{1}, \mathbf{x}_{2}, \mathbf{x}_{3}, \mathbf{c}\right)=$ $3 x_{t}+c \cdot 43$

Table 3.1 exhibits the estimates that result from imposing the restrictions using the optimal minimum distance estimator. 44 we also give the conventional generalized least squares estimates. They are minimum distance estimates in which the weighting matrix ( $\mathbf{A N}_{\mathbf{N}}$ ) is the inverse of

$$
\hat{\Omega}_{s}=\frac{1}{N} \sum_{i=1}^{N}\left(y_{i}-\hat{\Pi} x_{i}\right)\left(y_{i}-\prod_{\sim} x_{i}\right)^{\prime} \Theta\left(\frac{1}{N} \sum_{i=1}^{N} x_{i} x_{i}^{\prime}\right)^{-1} .
$$

We give the conventional standard errors based on $\left(F^{\prime} \hat{\Omega}_{\sim \mathbf{S}}^{-1} F\right)$ and the standard errors calculated according to Proposition 2. which do not require an assumption of homoskedastic linear regression. These standard errors are larger than the conventional ones, by about $30 \%$. The estimated gain in efficiency from using the appropriate metric is not very large; the standard errors calculated according to Proposition 2 are about $10 \%$ larger when we use conventional GLS instead of the optimum minimum distance estimator.

Table 3.1 also presents the estimated $\lambda$ 's. Consider, for example, an individual who was covered by collective bargaining in 1969. The linear predictor of $c$ increases by .089 if he is also covered in 1970, and it increases by an additional .036 if he is covered in all three years. The predicted $c$ for someone who is always covered is higher by .102 than for someone who is never covered.

Table 3.2 presents estimates under the constraint that $\lambda_{v}=0$. The increment in the distance statistic is $89.08-19.36=69.72$, which is a 
TABLE 3

RESTRICTED ESTIMATES

3.1

\begin{tabular}{|c|c|c|c|c|}
\hline & Coefficients & land Standard & Errors) & of: \\
\hline & Џ & SMSA & & RNS \\
\hline$\hat{B}:$ & $\begin{array}{l}.107 \\
(.016)\end{array}$ & $\begin{array}{l}.056 \\
(.020)\end{array}$ & & $\begin{array}{l}-.082 \\
(.045)\end{array}$ \\
\hline${ }_{\sim}^{\hat{B}}{ }_{\text {SLS }}:$ & $\begin{array}{l}.121 \\
(.013) \\
(.018)\end{array}$ & $\begin{array}{l}.050 \\
(.017) \\
(.021)\end{array}$ & & $\begin{array}{l}-.085 \\
(.040) \\
(.052)\end{array}$ \\
\hline
\end{tabular}

\begin{tabular}{|c|c|c|c|c|c|c|c|}
\hline & U1 & u. 2 & U3 & U1u2 & U103 & U2U3 & U1U2U3 \\
\hline \multirow[t]{3}{*}{$\underset{\sim}{\lambda}$} & $\begin{array}{r}-.02 \\
(.03:\end{array}$ & $\begin{array}{l}-.067 \\
(.040)\end{array}$ & $\begin{array}{l}-.082 \\
(.037)\end{array}$ & $\begin{array}{l}.156 \\
(.057)\end{array}$ & $\begin{array}{l}.152 \\
(.062)\end{array}$ & $\begin{array}{l}.195 \\
(.059)\end{array}$ & $\begin{array}{l}-.229 \\
(.085)\end{array}$ \\
\hline & SMSAI & SMSA2 & & SMSA3 3 & RNS1 & RNs2 & RNS3 \\
\hline & $\begin{array}{l}.086 \\
(.025)\end{array}$ & $\begin{array}{l}-.008 \\
(.046)\end{array}$ & & $\begin{array}{l}.032 \\
(.046)\end{array}$ & $\begin{array}{l}.100 \\
(.072)\end{array}$ & $\begin{array}{l}-.021 \\
(.077)\end{array}$ & $\begin{array}{l}-.128 \\
(.068)\end{array}$ \\
\hline
\end{tabular}

$x^{2}(23)=19.36$

3.2 Restrict $\lambda=0$.

\begin{tabular}{|c|c|c|c|c|c|}
\hline & Coefficients & land & Standard & Errors) & of: \\
\hline & $\cdot \mathrm{U}$ & & SMSA & & RNS \\
\hline$\hat{B}:$ & $\begin{array}{l}.157 \\
(.012)\end{array}$ & & $\begin{array}{l}.120 \\
(.013)\end{array}$ & & $\begin{array}{l}-.150 \\
(.016)\end{array}$ \\
\hline
\end{tabular}


yotes to Table 3:

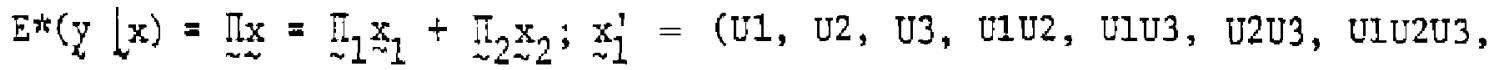
SMSA1, SMSA2, SMSA3, RNS1, RNS2, RNS3); $x_{2}^{\prime}=\left(1, S, \operatorname{EXP} 69, \operatorname{EXP} 69^{2}\right.$, FACE $)$.

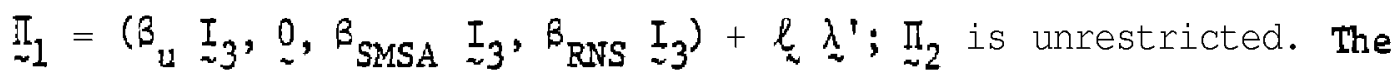
restric ions are expressed as $\pi=F \underset{\sim}{6}$, where $\delta$ is unrestricted. $\underset{\sim}{\hat{\beta}}$ and $\hat{\lambda}$ are minimum distance estimates with ${\underset{\sim}{A}}_{N}^{-1}=\hat{\Omega}$ in $(4.2) ; \hat{\theta}_{\text {GLS }}$ and $\hat{\lambda}_{\text {GLS }}$ are minfmum distance estimates with $\hat{\sim}_{N}^{\mathbf{- 1}}=\hat{\Omega}_{\boldsymbol{s}}$ in (5.1) $\hat{\lambda}_{\text {GLS }}$ is not shown in the table). The first standard error for $\hat{\hat{\beta}}_{\text {GLS }}$ is the conventional one based on $\left(\underline{F}_{\sim S}^{\prime} \hat{\Omega}^{-1} F\right)^{-1}$; the second standard error for $\hat{\beta}_{-G L S}$ is based on

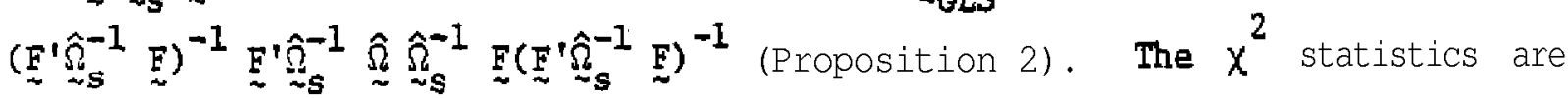

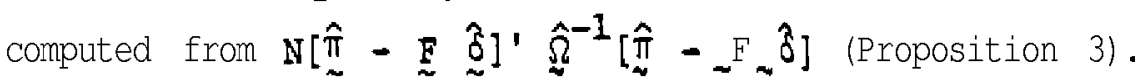


surprisingly large value to come from a $\chi^{2}$ (13) distribution. If we constrain only the union $\lambda^{\top} \mathrm{s}$ to be zero, then the increment is $57.06=19.36=37.7$, which is surprisingly large coning from a $x^{2}(7)$ distribution. So there is strong evidence for heterogeneity bias.

The union coefficient declines from.157 to .107 when we relax the $A_{*}=$ restriction. The least squares estimates for the separate cross sections, with no leads or lags, give union coefficients of .195, .189, and .191 in 1969, 1970, and 1971. 45 So the decline in the union coefficient, when we allow for heterogeneity bias, is $32 \%$ or $44 \%$ depending on which biased estimate (.16 or .19) one uses. The SMSA and region coefficients also decline in absolute value. The least squares estimates for the separate cross sections give an average SMSA coefficient of .147 and an average region coefficient of -.131. So the decline in the SMSA coefficient is either 53\% or 62\%, and the decline in absolute value of the region coefficient is either $45 \%$ or $37 \%$.

\subsection{Nonlinear Models: Labor Force Participation}

We shall illustrate some of the results in section 3 . The sample consists of 924 married women in the Michigan Panel study of Income Dynamics. The sample selection criteria and the means and standard deviations of the variables are in Table 4. Participation status is measured by the question "Did do any work for money last yeas?" We shall model participation

in 1968, 1970, 1972, and 1974.

In terms of the model described in Section 3.1, the wage predictors are schooling, experience, and experience squared, where experience is measured 
as age minus schooling minus six; the tastes for nonmarket time are predicted by these variables and by children. The specification for children is a conventional one that uses the number of children of age less than six (YS) and the total number of children in the family unit (K). ${ }^{46}$ Variables that affect only the lifetime budget constraint in this certainty model are captured by c. In particular, nonlabor income and the husband's wage are assumed to affect the wife's participation only through the lifetime budget constraint. The individual effect (c) will also capture unobserved permanent components in wages or in tastes for nonmarket time.

Table $\mathbf{S}$ presents maximum likelihood (vi) estimates of cross-section probit specifications for each of the four years. Table 6 presents unrestricted ML estimates for all lags and leads in $\mathbf{Y K}$ and $K$. If the residuals ( $\mathbf{u}_{\mathbf{i} t}$ ) in the latent variable model (3.1) have constant variance, then $a_{1} \ldots=a_{4} i n(\mathbf{3 . 8 )}$, and the submatrices of $\Pi$ corresponding to YK and $K$ should have the form $\beta I+\ell \underset{\sim}{\lambda^{\prime}}$. There may be some indication of this pattern in Table 6 , but it is much weaker than in the wage regressions in Table 2 .

we allow for unequal variances and provide formal tests by using the minimum distance estimator developed in Section 4.5. In Table 7.1 we impose the restrictions that

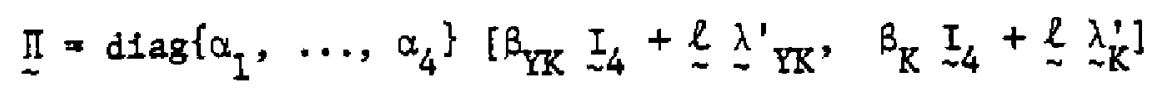

The minimum distance statistic is 53.8, which is a very surprising value coming from a $\chi^{2}(19)$ distribution. So the latent variable $c$ does not appear to provide an adequate interpretation of the unrestricted leads and lags. 
It may be that the distributed lag relationship between current participation and previous births is more general than the one implied by summing over the previous six years (YK) and over the previous eighteen years (K). It may be fruitful to explore this in more detail in future work. Perhaps strict exogeneity conditional on $\mathrm{c}$ will hold when we use a more general specification for lagged births. But we must keep in mind that this question is intrinsically tied to the functional form restrictions we saw in Section 3.3 that there always exist specifications in which $\mathbf{y}_{\mathbf{t}}$ is independent of $\mathbf{x}_{\mathbf{I}}, \ldots . \mathbf{x}_{\mathbf{T}}$ conditional on $\mathrm{c}$.

If we do impose the restrictions in Table 7.1 , then there is strong evidence that $\lambda \neq \underline{\sim}$. Constraining $\lambda=\underline{0}$ in Table 7.2 gives an increase in the distance statistic of $78.4-53.8=24.6$, which is surprisingly large to come from a $x^{2}(8)$ distribution.

In Table 7.3 we constrain all of the residual variances to be equal $\left(\alpha_{t}=1\right)$. An alternative interpretation of the time varying coefficients is provided in Table 7.4, where $\beta_{\mathrm{YK}}$ and $\beta_{\mathrm{K}}$ vary freely over time and $\boldsymbol{\alpha}_{\mathbf{t}} \mathbf{=} 1$. In principle. we could also allow the $\alpha_{t}$ to vary freely, since they can be identified from changes over time in the coefficients of $c$. In fact that model gives very imprecise results and it is difficult to ensure numerical accuracy.

We shall interpret the coefficients on $\mathbf{Y K}$ and $\mathrm{K}$ by following the procedure in (3.4). Table $\mathbf{8}$ presents estimates of the expected change in the participation probability when we assign an additional young child to a randomly chosen family, so that $Y K$ and $K$ increase by one. We compute this measure for the models in Tables 7.1, 7.3, and 7.4. The average change in 
the participation probability is $\mathbf{- . 0 9 6}$. We can get an indication of omitted variable bias by comparing these estimates with the ones based on Table 1.2, where $\lambda$ is constrained to be zero. Now the average change in the participation probability is $\mathbf{- . 1 2 2}$, so that the decline in absolute value when we control for $\mathrm{c}$ is 21\%. in alternative comparison can be based on the cross-section estimates, with no leads or lags, in Table 5. Now the average change in the participation probability is $\mathbf{- . 1 4 4}$, giving an omitted variable bias of $33 \%$.

Next we shall consider estimates from the logit framework of Section 3.2. Table 9 presents (standard) maximum likelihood estimates of cross-section logit specifications for each of the four years. We can use the cross-section probit results in Table 5 to ccistruct estimates of the expected change in the $\log$ odds of participation en we add a young child to a randomly chosen family. Doing this in each of the four years gives $-.502,-.598,-.683$, and -.703. With the logit estimates, we simply add together the coefficients on

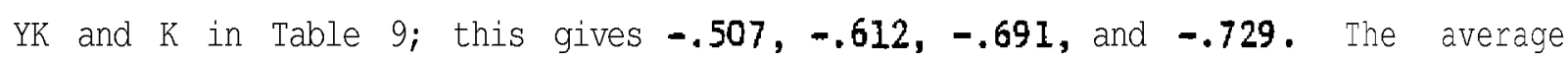
over the four years is $\mathbf{- . 6 2 1}$ for probit and $\mathbf{- . 6 3 5}$ for logit. so at this point there is little difference between the two functional forms.

Now allow for the latent variable (c). Table 10 presents the conditional maximum likelihood estimates for the fixed effects logit model. The striking result here is that, unlike the probit case, allowing for $c$ leads to an increase in the absolute value of the children coefficients. If we constrain $\beta_{\mathbf{Y K}}$ and $\beta_{\mathbf{K}}$ to be constant over time (Table 10.1), the estimated change in the log odds of participation when we add an additional young child is -.898. If we allow $\beta_{\mathbf{Y K}}$ and $\beta_{\mathbf{K}}$ to vary freely over time (Table 10.2), the average of 
the estimated changes is $\mathbf{- . 8 8 3}$. So the absolute value of the estimates increases by about $40 \%$ when we control for c using the logit framework. The estimation method is having a first order effect on the results.

It is commonly found that probit and logit specifications, when properly interpreted, give very similar results; our cross-section estimates are an example of this. But our attempt to incorporate latent variables has turned up marked differences between the probit and logit specifications. There are a number of possible explanations for this. The probit specification restricts $\mathbf{c}$ to have a normal distribution conditional on $\mathbf{x}$ with a linear regression function and constant variance. The conditional likelihood approach in the logit model does not impose this possibly false restriction. on the other hand, the probit model has a more general specification for the residual covariance matrix.

We have seen that the restrictions on the probit $\underset{\sim}{\operatorname{matrix}}$, which underlie our estimate of $\beta$, appear to be false. An analogous test in the logit framework is based on (3.10). We use conditional ML to estimate a model that includes $\mathrm{YR}_{s} \cdot \mathrm{D}_{\mathbf{t}}, \mathrm{K}_{\mathbf{s}} \cdot \mathrm{D}_{\mathrm{t}}(\mathbf{s}=1, \ldots, 4 ; \mathrm{t}=2,3,4)$, where $\mathrm{D}_{t}$ is a dummy variable that is one in period $t$ and zero otherwise. It is not restrictive :o exclude $\mathbf{Y R}_{\mathbf{g}} \cdot \mathbf{D}_{\mathbf{I}}$ and $\mathbf{K}_{\mathbf{s}} \cdot \mathbf{D}_{\mathbf{I}}$, since they can be absorbed in $\mathrm{C}$. We include 11so $D_{t}, S \cdot D_{t}, \operatorname{EXP} 68 \cdot D_{t}$, and $\operatorname{ExP} 68^{2} \cdot D_{t}(t=2,3,4)$. Then comparing the maximized sonditional likelihoods for this specification and the specification in Table 10.2 gives a conditional likelihood ratio statistic of 47.5 , which is a very surprising value to come from a $x^{2}(16)$ distribution. So the restrictions underlyfig our logit estimates of $B$ also appear to be false. 
It may be that the false restrictions simply imply different biases in the probit and logit specifications.

\section{CONCLUSION}

Our discussion has focused on models that are static conditional on a latent variable. The panel aspect of the data has primarily been used to control for the latent variable. Much work needs to be done on models that incorporate uncertainty and Interesting dynamics. Exploiting the mantingale implications of time-additive utility seems fruitful here, as' in Hall (1978) and Hansen and Singleton (1981). There is, however, a potentially important distinction between time averages and cross-section averages. A time average of forecast errors over $\mathrm{T}$ periods should converge to zero as $\mathrm{T}+\boldsymbol{\infty}$. But an average of forecast errors across $\mathrm{N}$ individuals surely need not converge to zero as $\mathrm{N}+\infty$; there my be common components in those errors, due to economy-wide innovations. The same point applies when we consider covariances of forecast errors with variables that are in the agents' information sets. If those conditioning variables are discrete, we can think of averaging over subsets of the forecast errors; as $\mathrm{T} \rightarrow \infty$, these averages should converge to zero, but not necessarily a.s $N \rightarrow \infty$. As for controlling for latent variables, I think that future work will have to address the lack of identification that we have uncovered. It is not restrictive to assert that $\left(\mathbf{y}_{1}, \ldots . . \mathbf{y}_{\mathrm{T}}\right)$ and $\left(\mathbf{x}_{\mathbf{1}}, \ldots . . \mathbf{x}_{\mathrm{T}}\right)$ are independent conditional on some latent variable $c$. 
T A B L E 4

\section{CUARACTERTSTICS OF UICHIGAN PANEL STUDY OF INCOME DYNAMTCS MARRIED DOUEN}

Means and Standard Deviations

$$
N=924
$$

\begin{tabular}{|c|c|c|}
\hline Variable & Mean & Standard Deviation \\
\hline $\begin{array}{l}\text { LFP1 } \\
\text { LFP2 } \\
\text { LFP3 } \\
\text { LFP } 4\end{array}$ & $\begin{array}{l}.499 \\
.530 \\
.529 \\
.566\end{array}$ & \\
\hline $\begin{array}{l}\text { YK1 } \\
\text { YK2 } \\
\text { YK3 } \\
\text { YK } 4\end{array}$ & $\begin{array}{c}.969 \\
.764 \\
.551 \\
.363\end{array}$ & $\begin{array}{r}1.200 \\
1.069 \\
.895 \\
.685\end{array}$ \\
\hline $\begin{array}{l}\mathrm{K} 1 \\
\mathrm{~K} 2 \\
\mathrm{~K} 3 \\
\mathrm{~K} 4\end{array}$ & $\begin{array}{l}2.38 \\
2.30 \\
2.11 \\
1.84\end{array}$ & $\begin{array}{l}1.69 \\
1.64 \\
1.61 \\
1.52\end{array}$ \\
\hline $\begin{array}{l}S \\
\operatorname{EXP} 68 \\
\operatorname{EXP} 68^{2}\end{array}$ & $\begin{array}{l}12.1 \\
17.2 \\
368 .\end{array}$ & $\begin{array}{l}2.1 \\
8.5 \\
301 .\end{array}$ \\
\hline
\end{tabular}

Notes to Table 4:

LFP1, . . . LFP4 - 1 if answered "yes" to "Did work for money last year, 0 otherwise, referring to 1968, 1970, 1972, 1974; YK1, . . . . YK4 - number of children of age less than six in 1968, 1970, 1972, $1974 ; \mathrm{K} 1, \ldots, \mathrm{K} 4$ - number of children of age less than eighteen living in the family unit in 1968, 1970, 1972, 1974; S -- years of schooling completed; EXP68 -- (age in 1968 - S-6). The sample selection criteria required that the women be married to the same spouse from 1968 to 1976; not part of the low income subsample; between 20 and 50 
years old in 1968; white; out of school from 1968 to 1076; not disabled. We required complete data on the variables in the Table, and that there be no inconsistency between reported earnings and the answer to the participation question. 
TABLE 5

ML PROBIT CROSS-SECTION ESTIMATES

\begin{tabular}{|c|c|c|c|c|c|c|c|c|}
\hline \multirow{2}{*}{$\begin{array}{l}\text { Dependent } \\
\text { Variable }\end{array}$} & \multicolumn{3}{|c|}{ Coefficients } & \multirow{2}{*}{$\frac{\text { Standard }}{\mathrm{YK} 4}$} & \multirow{2}{*}{$\begin{array}{c}\text { Errors) } \\
\text { KI }\end{array}$} & \multicolumn{3}{|l|}{ of: } \\
\hline & $Y K I$ & YK2 & YK3 & & & $\mathrm{k} 2$ & K3 & K 4 \\
\hline LFP1 & $\begin{array}{l}-. .246 \\
(.046)\end{array}$ & " & $=$ & - & $\begin{array}{l}-.063 \\
(.031)\end{array}$ & - & - & \\
\hline LFP2 & & $\begin{array}{l}-.293 \\
(.055)\end{array}$ & - & & & $\begin{array}{l}-.075 \\
(.031)\end{array}$ & - & - \\
\hline IFP3 & & & $\begin{array}{c}-.342 \\
(.067)\end{array}$ & - & & & $\begin{array}{l}-.077 \\
(.032)\end{array}$ & - \\
\hline IFP4 & & & & $\begin{array}{l}-.366 \\
(.081)\end{array}$ & - & & & $\begin{array}{c}-.069 \\
(.034)\end{array}$ \\
\hline
\end{tabular}

HOTES TO TABLE 5:

separate $\mathbf{I L}$ estimates each year. AII specifications include (1, S, $\left.\operatorname{EXP} 68, \operatorname{EXP} 68^{2}\right)$. 
TABLE 6

UINESTRICTED ML PROBIT ESTIMATES

\begin{tabular}{|c|c|c|c|c|c|c|c|c|}
\hline \multirow{2}{*}{$\begin{array}{l}\text { Dependent } \\
\text { Variable }\end{array}$} & \multicolumn{3}{|c|}{ Coefficients land } & Standard & Errors) & \multicolumn{2}{|l|}{ of: } & \multirow[b]{2}{*}{ K4 } \\
\hline & YK1 & $\mathrm{YK} 2$ & YK3 & YK 4 & $\mathrm{~K} 1$ & $\mathrm{~K} 2$ & K3 & \\
\hline LFP1 & $\begin{array}{l}-.205 \\
(.081)\end{array}$ & $\begin{array}{l}-.017 \\
(.119)\end{array}$ & $\begin{array}{l}-.160 \\
(.141)\end{array}$ & $\begin{array}{l}.420 \\
(.144)\end{array}$ & $\begin{array}{c}.176 \\
(.076)\end{array}$ & $\begin{array}{l}-.142 \\
(.100)\end{array}$ & $\begin{array}{l}-.196 \\
(.110)\end{array}$ & $\begin{array}{l}.063 \\
(.090)\end{array}$ \\
\hline LFP2 & $\begin{array}{l}-.047 \\
(.079)\end{array}$ & $\begin{array}{l}-.238 \\
(.117)\end{array}$ & $\begin{array}{c}-.047 \\
(.140)\end{array}$ & $\begin{array}{l}.093 \\
(.142)\end{array}$ & $\begin{array}{l}.320 \\
(.077)\end{array}$ & $\begin{array}{l}-.278 \\
(.102)\end{array}$ & $\begin{array}{l}-.250 \\
(.110)\end{array}$ & $\begin{array}{l}.177 \\
(.090)\end{array}$ \\
\hline LFP 3 & $\begin{array}{l}-.254 \\
(.080)\end{array}$ & $\begin{array}{l}.214 \\
(.116)\end{array}$ & $\begin{array}{l}-.190 \\
(.139)\end{array}$ & $\begin{array}{l}-.209 \\
(.141)\end{array}$ & $\begin{array}{c}.204 \\
(.077)\end{array}$ & $\begin{array}{c}-.210 \\
(.102)\end{array}$ & $\begin{array}{l}-.045 \\
(.112)\end{array}$ & $\begin{array}{l}.030 \\
(.090)\end{array}$ \\
\hline $\mathrm{LEP} 4$ & $\begin{array}{l}-.195 \\
(.079)\end{array}$ & $\begin{array}{l}.252 \\
(.118)\end{array}$ & $\begin{array}{l}-.211 \\
(.139)\end{array}$ & $\begin{array}{l}-.282 \\
(.138)\end{array}$ & $\begin{array}{l}.20 \\
(. .175)\end{array}$ & $\begin{array}{l}.083 \\
(.100)\end{array}$ & $\begin{array}{l}-.181 \\
(.110)\end{array}$ & $\begin{array}{l}.058 \\
(.090)\end{array}$ \\
\hline
\end{tabular}

WOTES TO TABLE 6:

Separate ML estimates each year. All specifications include (1, S, ExP68, $\left.\operatorname{EXP} 68^{2}\right)$ 